\title{
Structural Evidence That Alkoxy Substituents Adopt Electronically Preferred Pseudoaxial Orientations in Six-Membered Ring Dioxocarbenium Ions
}

\author{
Stephen Chamberland, Joseph W. Ziller, and K. A. Woerpel \\ Department of Chemistry, University of California, \\ Irvine, California 92697-2025 \\ Supporting Information
}

I. General Information

II. Synthesis of Lactones

$\begin{array}{lll}\text { A. } & \text { Synthesis of 5-(Benzyloxy)tetrahydropyran-2-one (S3) } & \text { S-2 } \\ \text { B. } & \text { Synthesis of 5-Methyltetrahydropyran-2-one (S4) } & \text { S-5 } \\ \text { C. } & \text { Synthesis of 5-Methoxytetrahydropyran-2-one (S6) } & \text { S-6 }\end{array}$

III. Preparation of New Lactonium Salts

A. 4-(Benzyloxy)-1-ethoxy-2,3,4,5-tetrahydropyrylium hexachloroantimonate (5)

1-Ethoxy-4-methyl-2,3,4,5-tetrahydropyrylium hexachloroantimonate (6) S-8

C. 1-Ethoxy-4-methoxy-2,3,4,5-tetrahydropyrylium hexachloroantimonate (S7) S-10

IV. Proofs of Structure, Conformation, and Stereochemistry

A. Preferred Pseudoaxial Orientation is Independent of Solvent for S3 $\quad$ S-11

B. X-ray Crystallographic Data for $5 \quad$ S-13

C. X-ray Crystallographic Data for $9 \quad$ S-22

D. Relevant ${ }^{1} \mathrm{H}$ NMR Coupling Constant Data for 5, 6, S7, and $10 \quad$ S-36

V. Computational Studies on 4-Methoxy- and 4-Methyl-2,3,4,5-tetrahydropyrylium Ions S-38

$\begin{array}{lr}\text { VI. Bibliography } & \text { S-52 }\end{array}$

VII. Analytical Data

${ }^{1} \mathrm{H},{ }^{13} \mathrm{C}$ NMR Spectra of Relevant Compounds and of 5, 6, S7, and $10 \quad S-53$

\section{General Information}

${ }^{1} \mathrm{H}$ and ${ }^{13} \mathrm{C}$ NMR spectra were recorded at ambient temperature at $500 \mathrm{MHz}$ and 125

MHz, respectively, using Bruker DRX 500 spectrometers. All chemical shifts are reported in ppm relative to tetramethylsilane on the $\delta$ scale (or, where noted, to residual solvent peak), multiplicity $(\mathrm{br}=$ broad, $\mathrm{s}=$ singlet, $\mathrm{d}=$ doublet, $\mathrm{t}=$ triplet, $\mathrm{q}=$ quartet, quint $=$ quintet, and $\mathrm{m}=$ multiplet), coupling constants in $\mathrm{Hz}$, and integration. When proton-carbon connectivity assignments are noted, data was confirmed using 2D-COSY $\left({ }^{1} \mathrm{H}^{-1} \mathrm{H}\right) \mathrm{NMR}$ analysis. Infrared (IR) spectra were obtained using a MIDAC Prospect FT-IR spectrometer. High resolution mass 
spectra were acquired on a VG Analytical 7070E or Fisons Autospec spectrometer, and were obtained by peak matching. Elemental analyses were performed by Atlantic Microlab, Atlanta, GA. Melting points are uncorrected. Liquid chromatography was performed using variable forced air flow (flash chromatography) of the indicated solvent system or solvent gradient on 60 $\AA$ SiliTech or EM Reagents silica gel $\left(\mathrm{SiO}_{2}\right)(60 \AA$, 230-400 mesh). All reaction mixtures not containing aqueous reagents were carried out under an atmosphere of dry nitrogen using standard syringe/septa techniques. Glassware was oven-dried, and then both flame-dried and cooled to ambient temperature under a stream of dry nitrogen or under vacuum. Unless otherwise noted, all reagents were used as received from commercial suppliers. Triethyloxonium hexachloroantimonate (Aldrich) was recrystallized twice from $\mathrm{CH}_{2} \mathrm{Cl}_{2}$ to give white needles and was stored at $-20{ }^{\circ} \mathrm{C}$ in a nitrogen atmosphere dry box. Tetrahydrofuran, diethyl ether, and dichloromethane were dried by filtration through alumina according to the procedure of Grubbs. ${ }^{1}$

\section{Synthesis of Lactones}

IIA. Synthesis of 5-(Benzyloxy)tetrahydropyran-2-one (S3)

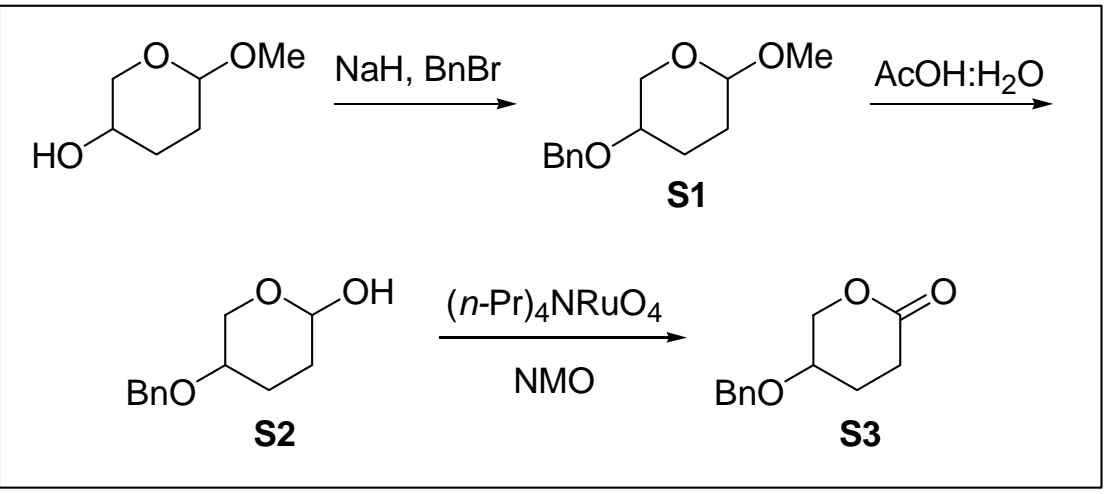




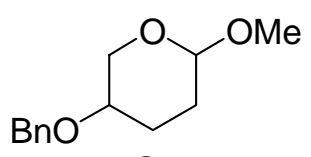

S1

5-Benzyloxy-2-methoxytetrahydropyran (S1): To a suspension of $\mathrm{NaH}(0.162 \mathrm{~g}, 6.75 \mathrm{mmol})$ in $5 \mathrm{~mL}$ of anhydrous DMF was added 2-methoxytetrahydropyran-5-ol ${ }^{2}(0.595 \mathrm{~g}, 4.50 \mathrm{mmol})$ dropwise by syringe. The mixture was stirred for $5 \mathrm{~min}$, and then $\mathrm{BnBr}(0.64 \mathrm{~mL}, 5.4 \mathrm{mmol})$ was added by syringe. After $12 \mathrm{~h}$, the mixture was partitioned between $10 \mathrm{~mL}$ of methyl tertbutyl ether (MTBE) and $5 \mathrm{~mL}$ of $\mathrm{H}_{2} \mathrm{O}$, and the layers were separated. The aqueous portion was extracted $3 \times 3 \mathrm{~mL}$ of MTBE. The combined organic extracts were washed with $3 \times 2 \mathrm{~mL}$ of $\mathrm{H}_{2} \mathrm{O}$ and $1 \times 5 \mathrm{~mL}$ of $1.0 \mathrm{M} \mathrm{HCl}$, and were dried over anhydrous $\mathrm{MgSO}_{4}$, filtered, and concentrated in vacuo. Purification by flash column chromatography on silica gel (0:100 to 20:80 EtOAc/hexanes) afforded the product as a colorless liquid and as a 1:0.4 mixture of $\operatorname{anomers}^{3}(0.747 \mathrm{~g}, 75 \%)$. The spectral data correlates with the previously reported data for $\mathbf{S 1}:^{4}$ ${ }^{1} \mathrm{H}$ NMR (500 MHz, $\left.\mathrm{CDCl}_{3}\right)$ $\delta$ 7.35-7.25 (m, 7H), 4.61-4.53 (m, 4.2H), 3.87 (dd, $J=11.9,2.5$, 1H), $3.66(\mathrm{ddd}, J=10.8,4.5,1.7,0.4 \mathrm{H}), 3.58(\mathrm{ddd}, J=11.9,4.1,1.5,1 \mathrm{H}), 3.52-3.44(\mathrm{~m}, 1.4 \mathrm{H})$, $3.40(\mathrm{~s}, 3 \mathrm{H}), 3.36(\mathrm{~s}, 1.2 \mathrm{H}), 2.06(\mathrm{tt}, J=10.4,3.3,0.4 \mathrm{H}), 2.00(\mathrm{tt}, J=10.4,3.8,1 \mathrm{H}), 1.90(\mathrm{~m}$, $0.4 \mathrm{H}), 1.83(\mathrm{~m}, 1 \mathrm{H}), 1.74-1.67(\mathrm{~m}, 1.4 \mathrm{H}), 1.63(\mathrm{~m}, 0.4 \mathrm{H}), 1.53(\mathrm{~m}, 1 \mathrm{H}) ;{ }^{13} \mathrm{C}$ NMR $(125 \mathrm{MHz}$ $\left.\mathrm{CDCl}_{3}\right) \delta 138.6,128.4,127.6,99.4,97.7,72.3,71.4,70.42,70.36,63.1,62.6,55.3,54.7,31.6$ 28.5, 26.2, 25.0, 23.6.

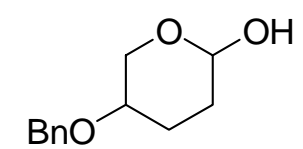

5-(Benzyloxy)tetrahydropyran-2-ol (S2): ${ }^{\text {S2 }^{2}}$ A $\quad$ solution of $\quad$ 5-(benzyloxy)-2methoxytetrahydropyran $(\mathbf{S 1})(0.747 \mathrm{~g}, 3.36 \mathrm{mmol})$ in $68 \mathrm{~mL}$ of a $2: 1(\mathrm{v} / \mathrm{v})$ mixture of HOAc 
and $\mathrm{H}_{2} \mathrm{O}$ was heated at $50-55{ }^{\circ} \mathrm{C}$ for $12 \mathrm{~h}$. The solution was diluted with $50 \mathrm{~mL}$ of MTBE and the layers were separated. The aqueous phase was extracted with $6 \times 10 \mathrm{~mL}$ of MTBE. The combined organic extracts were washed with saturated aqueous $\mathrm{NaHCO}_{3}$ until bubbling ceased. They were dried over anhydrous $\mathrm{MgSO}_{4}$, filtered, and concentrated in vacuo to afford a colorless oil of sufficient purity to carry on without further manipulation as a 1:1 mixture of anomers ${ }^{3}$ $(0.614 \mathrm{~g}, 88 \%): \mathrm{R}_{f}=0.52,60: 40$ EtOAc/hexanes; ${ }^{1} \mathrm{H}$ NMR $\left(500 \mathrm{MHz}, \mathrm{CDCl}_{3}\right) \delta 7.30(\mathrm{~m}, 10 \mathrm{H})$ $5.03(\mathrm{dd}, J=5.8,2.7,1 \mathrm{H}), 5.01(\mathrm{t}, J=3.7,1 \mathrm{H}), 4.60(\mathrm{~d}, J=12.0,1 \mathrm{H}), 4.59(\mathrm{~d}, J=12.0,1 \mathrm{H})$, $4.56(\mathrm{~d}, J=12.0,1 \mathrm{H}), 4.54(\mathrm{~d}, J=12.0,1 \mathrm{H}), 4.07$ (ddd, $J=11.4,2.8,0.8,1 \mathrm{H}), 3.91(\mathrm{dd}, J=$ 11.4, 7.3, 1H), $3.67(\mathrm{ddd}, J=11.3,3.2,0.7,1 \mathrm{H}), 3.49(\mathrm{~m}, 3 \mathrm{H}), 2.54(\mathrm{br} \mathrm{s}, 2 \mathrm{H}), 2.08(\mathrm{~m}, 1 \mathrm{H})$, $1.85(\mathrm{~m}, 2 \mathrm{H}), 1.95(\mathrm{~m}, 1 \mathrm{H}), 1.71(\mathrm{~m}, 2 \mathrm{H}), 1.52(\mathrm{~m}, 2 \mathrm{H}) ;{ }^{13} \mathrm{C}$ NMR $\left(125 \mathrm{MHz}, \mathrm{CDCl}_{3}\right) \delta 138.44$ 138.40, 128.40, 128.39, 127.63, 127.61, 127.58, 127.57, 93.7, 92.6, 71.5, 71.4, 70.5, 70.3, 64.7, 28.8, 28.0, 24.9, 24.5; IR (thin film) 3396 (br), 3030, 2947, 1454, 1350, $1076 \mathrm{~cm}^{-1}$; HRMS $\left(\mathrm{CI} / \mathrm{NH}_{3}\right) \mathrm{m} / \mathrm{z}$ calcd for $\mathrm{C}_{12} \mathrm{H}_{16} \mathrm{O}_{3}\left(\mathrm{M}^{+}\right)$208.1099, found 208.1103 .

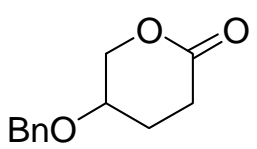

S3

5-(Benzyloxy)tetrahydropyran-2-one $\quad(\mathbf{S 3}):^{5}$ To a cooled $\left(0{ }^{\circ} \mathrm{C}\right)$ mixture of $N$ methylmorpholine- $N$-oxide (0.853 g, $7.28 \mathrm{mmol}), 5$-(benzyloxy)tetrahydropyran-2-ol (S2) (1.01 g, $4.85 \mathrm{mmol}$ ), and approximately $100 \mathrm{mg}$ of activated $3 \AA$ molecular sieves in $35 \mathrm{~mL}$ of $\mathrm{CH}_{2} \mathrm{Cl}_{2}$ was added tetra- $n$-propylammonium perruthenate $(0.083 \mathrm{~g}, 0.24 \mathrm{mmol})$. The reaction flask was protected from light. The mixture was stirred for $1 \mathrm{~h}$ with warming to ambient temperature, and then it was then filtered and concentrated in vacuo. Purification by flash column chromatography on silica gel (50:50 EtOAc/hexanes) afforded the product as a colorless oil 
$(0.806 \mathrm{~g}, 81 \%)$. We have observed that $\delta$-valerolactones can decompose upon standing, so they should be used without undue delay: $\mathrm{R}_{f}=0.43,60: 40$ EtOAc/hexanes; ${ }^{1} \mathrm{H}$ NMR $(500 \mathrm{MHz}$, $\left.\mathrm{CDCl}_{3}\right) \delta 7.35(\mathrm{~m}, 5 \mathrm{H}), 4.60(\mathrm{~d}, J=12.5,1 \mathrm{H}), 4.57(\mathrm{~d}, J=12.5,1 \mathrm{H}), 4.45(\mathrm{ddd}, J=12.1,4.1$, $1.9,1 \mathrm{H}), 4.32(\mathrm{dd}, J=12.1,4.1,1 \mathrm{H}), 3.85$ (quint, $J=4.1,1 \mathrm{H}), 2.76(\mathrm{ddd}, J=17.5,10.0,7.2$, 1H), 2.53 (ddd, $J=17.5,6.7,4.8,1 \mathrm{H}), 2.13$ (ddddd, $J=18.0,7.2,4.8,4.1,1.9,1 \mathrm{H}), 2.13$ (dddd, $J=18.0,10.0,6.7,4.1,1 \mathrm{H}) ;{ }^{13} \mathrm{C} \mathrm{NMR}\left(125 \mathrm{MHz}, \mathrm{CDCl}_{3}\right) \delta 170.5,137.5,128.6,128.0,127.6$, 70.7, 70.5, 69.0, 26.3, 24.6; IR (thin film) 3063, 2873, 1732, 1241, 1170, $1102 \mathrm{~cm}^{-1}$; HRMS (EI) $m / z$ calcd for $\mathrm{C}_{12} \mathrm{H}_{14} \mathrm{O}_{3}\left(\mathrm{M}^{+}\right)$206.0943, found 206.0943.

\section{IIB. Synthesis of 5-Methyltetrahydropyran-2-one (S4)}
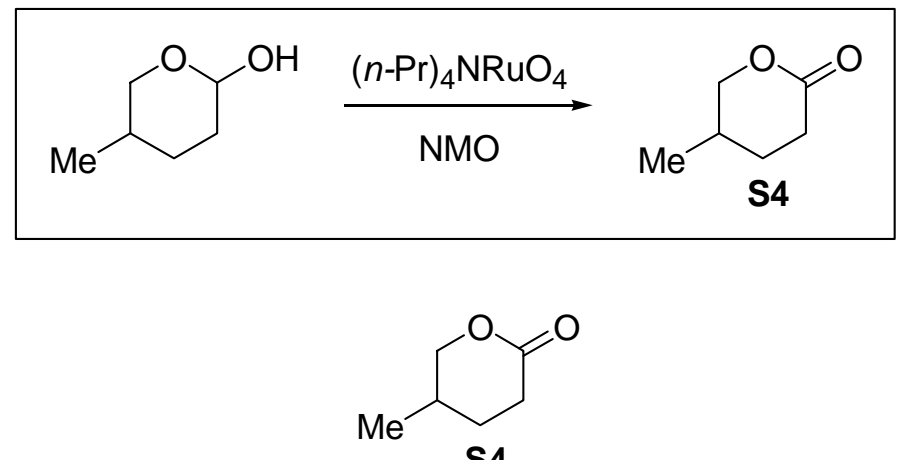

5-Methyltetrahydropyran-2-one (S4): ${ }^{5}$ To a mixture of $N$-methylmorpholine- $N$-oxide $(1.27 \mathrm{~g}$, $10.9 \mathrm{mmol}), 5$-methyltetrahydropyran-2-ol ${ }^{4} \quad(0.84 \mathrm{~g}, 7.2 \mathrm{mmol})$, and approximately $100 \mathrm{mg}$ of activated $4 \AA$ molecular sieves in $40 \mathrm{~mL}$ of $\mathrm{CH}_{2} \mathrm{Cl}_{2}$ was added tetra- $n$-propylammonium perruthenate $(0.127 \mathrm{~g}, 0.362 \mathrm{mmol})$. The reaction flask was protected from light and the mixture was stirred for $1 \mathrm{~h}$ at ambient temperature. The mixture was then filtered and concentrated in vacuo. Purification by flash column chromatography on deactivated silica gel (50:50 $\mathrm{Et}_{2} \mathrm{O} /$ pentane $)$ afforded the product as a colorless oil $(0.673 \mathrm{~g}, 81 \%)$. The spectral data correlates with the previously reported data for $\mathbf{S 4}{ }^{6}$ We have observed that $\delta$-valerolactones can decompose upon standing, so they should be used without undue delay: ${ }^{1} \mathrm{H}$ NMR (500 MHz, 
$\left.\mathrm{CDCl}_{3}\right) \delta 4.32(\mathrm{ddd}, J=11.1,4.6,2.1,1 \mathrm{H}), 3.91(\mathrm{dd}, J=11.1,10.1,1 \mathrm{H}), 2.64(\mathrm{ddd}, \mathrm{J}=17.9$, 6.9, 4.2, 1H), $2.51(\mathrm{ddd}, \mathrm{J}=17.9,10.0,7.4,1 \mathrm{H}), 2.06(\mathrm{qdq}, J=10.0,6.8,4.7,1 \mathrm{H}), 1.98$ (ddddd, $J=13.5,7.4,4.7,4.2,2.1,1 \mathrm{H}), 1.54(\mathrm{dtd}, J=13.5,10.0,6.9,1 \mathrm{H}), 1.01(\mathrm{~d}, J=6.8,3 \mathrm{H}) ;{ }^{13} \mathrm{C}$ NMR (125 MHz, $\left.\mathrm{CDCl}_{3}\right) \delta 171.1,74.9,29.1,27.9,27.5,16.5$; IR (thin film) 3059, 2962, 1736, 1460, 1341, $1051 \mathrm{~cm}^{-1}$; HRMS (EI/GCMS) $m / z$ calcd for $\mathrm{C}_{6} \mathrm{H}_{10} \mathrm{O}_{2}\left(\mathrm{M}^{+}\right)$114.0681, found 114.0680.

IIC. Synthesis of 5-Methoxytetrahydropyran-2-one (S6)

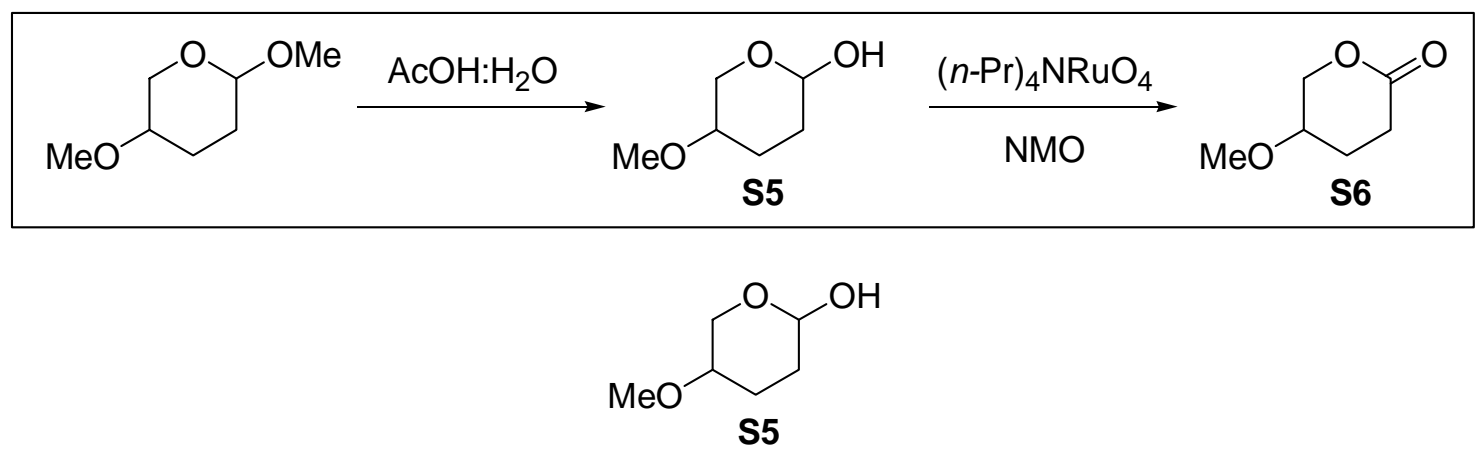

5-Methoxytetrahydropyran-2-ol (S5): ${ }^{5}$ A solution of 2,5-dimethoxytetrahydropyran ${ }^{7}$ (5.809 g, $39.75 \mathrm{mmol})$ in $380 \mathrm{~mL}$ of a 2:1 (v/v) mixture of $\mathrm{HOAc}$ and $\mathrm{H}_{2} \mathrm{O}$ was heated at $50-55^{\circ} \mathrm{C}$ for 12 h. The solution was diluted with $400 \mathrm{~mL}$ of MTBE and the layers were separated. The aqueous layer was extracted with $6 \times 30 \mathrm{~mL}$ of MTBE. The combined organic extracts were washed with saturated aqueous $\mathrm{NaHCO}_{3}$ until bubbling had ceased. They were dried over anhydrous $\mathrm{MgSO}_{4}$, filtered, and concentrated in vacuo to afford a colorless oil of sufficient purity to carry on without further manipulation as a 1:1 mixture of anomers ${ }^{3,8}(2.043 \mathrm{~g}, 39 \%): \mathrm{R}_{f}=0.25,75: 25$ EtOAc/hexanes; ${ }^{1} \mathrm{H}$ NMR $\left(500 \mathrm{MHz}, \mathrm{CDCl}_{3}\right) \delta 5.05(\mathrm{dd}, J=7.4,4.6,1 \mathrm{H}), 4.98(\mathrm{dd}, J=7.9$, $3.2,1 \mathrm{H}), 4.07(\mathrm{ddd}, J=11.7,2.9,1.0,1 \mathrm{H}), 3.89(\mathrm{dd}, J=11.7,6.5,1.1,1 \mathrm{H}), 3.65(\mathrm{ddd}, J=11.7$, 3.4, 1.1, 1H), $3.51(\mathrm{ddd}, J=11.7,5.5,1.3,1 \mathrm{H}), 3.37(\mathrm{~s}, 6 \mathrm{H}), 3.30(\mathrm{~m}, 1 \mathrm{H}), 3.26(\mathrm{~m}, 1 \mathrm{H}), 2.08$ (ddtd, $J=12.4,10.5,3.4,1.0,1 \mathrm{H}), 2.00(\mathrm{ddtd}, J=12.4,9.3,4.1,3.1,1 \mathrm{H}), 1.90(\mathrm{~m}, 1 \mathrm{H}), 1.82(\mathrm{~m}$, 
1H), $1.79(\mathrm{~m}, 1 \mathrm{H}), 1.64(\mathrm{~m}, 1 \mathrm{H}), 1.51(\mathrm{~m}, 1 \mathrm{H}) ;{ }^{13} \mathrm{C} \mathrm{NMR}\left(125 \mathrm{MHz}, \mathrm{CDCl}_{3}\right) \delta$ 93.4, 92.9, 73.53, 73.50, 64.1, 63.8, 56.3, 56.1, 28.6, 27.4, 24.6, 23.6; IR (thin film) 3393, 2945, 1651, 1455, 1274 , $1099 \mathrm{~cm}^{-1}$; HRMS (EI+) $\mathrm{m} / z$ calcd for $\mathrm{C}_{6} \mathrm{H}_{12} \mathrm{O}_{3}\left(\mathrm{M}^{+}\right)$132.0786, found 132.0784 .

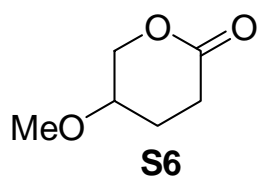

5-Methoxytetrahydropyran-2-one (S6): ${ }^{5}$ To a mixture of $N$-methylmorpholine- $N$-oxide $(2.72$ g, $23.2 \mathrm{mmol}$ ), 5-methoxytetrahydropyran-2-ol (S5) (2.04 g, $15.5 \mathrm{mmol})$, and approximately 100 mg of activated $4 \AA$ molecular sieves in $110 \mathrm{~mL}$ of $\mathrm{CH}_{2} \mathrm{Cl}_{2}$ was added tetra- $n$-propylammonium perruthenate $(0.272 \mathrm{~g}, 0.773 \mathrm{mmol})$. The reaction flask was protected from light and the mixture was stirred for $1 \mathrm{~h}$ at ambient temperature. The mixture was then filtered and concentrated in vacuo. Purification by flash column chromatography on silica gel (50:50 EtOAc/hexanes) afforded the product as a colorless oil $(1.45 \mathrm{~g}, 72 \%)$. We have observed that $\delta$-valerolactones can decompose upon standing, so they should be used without undue delay: ${ }^{1} \mathrm{H}$ NMR $(500 \mathrm{MHz}$, $\left.\mathrm{CDCl}_{3}\right) \delta 4.42(\mathrm{ddd}, J=12.2,3.8,2.1,1 \mathrm{H}), 4.30(\mathrm{ddd}, J=12.2,3.8,1 \mathrm{H}), 3.65$ (quint, $J=3.8$, 1H), 3.39 (s, 3H), $2.70(\mathrm{ddd}, J=17.5,9.8,7.4,1 \mathrm{H}), 2.51(\mathrm{ddd}, J=17.5,6.4,5.0,1 \mathrm{H}), 2.06(\mathrm{~m}$, 2H); ${ }^{13} \mathrm{C}$ NMR (125 MHz, $\left.\mathrm{CDCl}_{3}\right) \delta 170.5,71.1,70.2,56.1,26.1,24.1$; IR (thin film) 2935, 1740, 1461, 1346, 1244, $1065 \mathrm{~cm}^{-1}$; HRMS $\left(\mathrm{CI} / \mathrm{NH}_{3}\right) \mathrm{m} / z$ calcd for $\mathrm{C}_{6} \mathrm{H}_{10} \mathrm{O}_{3}\left(\mathrm{M}^{+}\right)$130.0630, found 130.0629 .

\section{Preparation of Lactonium Ion Salts}

General observations and comments about lactonium ion salts: These salts are known to decompose over several days, ${ }^{9}$ so they should be stored in a nitrogen atmosphere dry box at -20 ${ }^{\circ} \mathrm{C}$. They will decompose within seconds in air and are known to be very sensitive to 
nucleophiles. ${ }^{10,11}$ Solutions of these salts in $\mathrm{CD}_{2} \mathrm{Cl}_{2}$ under nitrogen in a J. Young tube have darkened upon standing overnight at ambient temperature. Spectral analysis of these darkened solutions indicated that significant decomposition occurred.

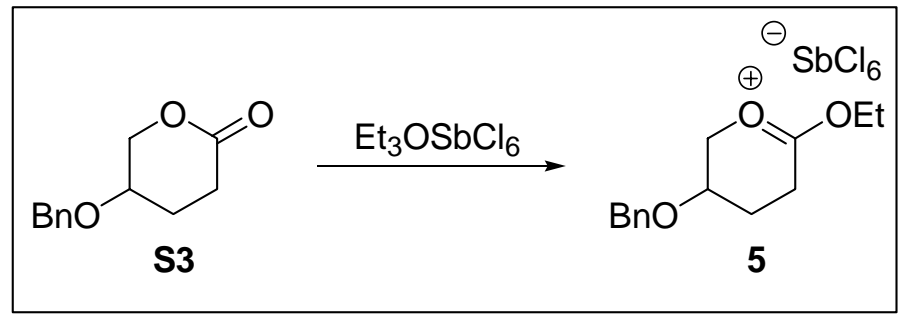

\section{IIIA. 4-(Benzyloxy)-1-ethoxy-2,3,4,5-tetrahydropyrylium hexachloroantimonate}

(5): To a 10-mL Schlenk flask containing a solution of freshly prepared 5(benzyloxy)tetrahydropyran-2-one $(\mathbf{S 3})(0.042 \mathrm{~g}, 0.20 \mathrm{mmol})$ in $2.5 \mathrm{~mL}$ of $\mathrm{CH}_{2} \mathrm{Cl}_{2}$ was added triethyloxonium hexachloroantimonate $(0.089 \mathrm{~g}, 0.20 \mathrm{mmol})$. The flask was protected from light, and the colorless mixture was stirred at ambient temperature in a nitrogen atmosphere dry box for $3 \mathrm{~h}$. Anhydrous $\mathrm{Et}_{2} \mathrm{O}$ was added dropwise until the solution became translucent, but without the occurrence of precipitation. Then, the mixture was transferred to a $-20{ }^{\circ} \mathrm{C}$ freezer to induce crystallization. After $18 \mathrm{~h}$ the supernatant was removed. The resulting solid was washed twice with $3 \mathrm{~mL}$ a 5:1 mixture of anhydrous $\mathrm{Et}_{2} \mathrm{O}: \mathrm{CH}_{2} \mathrm{Cl}_{2}$, and then was dried under vacuum to provide the crystalline product (colorless needles) $(0.049 \mathrm{~g}, 42 \%):{ }^{1} \mathrm{H} \mathrm{NMR}\left(500 \mathrm{MHz}, \mathrm{CD}_{2} \mathrm{Cl}_{2}\right.$, referenced to $\delta 5.32) \delta 7.41-7.35\left(\mathrm{~m}, 5 \mathrm{H},-\mathrm{OCH}_{2} \mathrm{C}_{6} H_{5}\right), 5.32(\mathrm{ddd}, J=12.3,2.6,2.0,1 \mathrm{H} ; J$ values determined by Gaussian multiplication $\left.(\mathrm{LB}=-3.0 \mathrm{~Hz}, \mathrm{~GB}=0.1 \mathrm{~Hz}), \mathrm{C} 5-H_{\mathrm{eq}}\right), 5.25(\mathrm{dd}$, $J=12.3,2.0,1 \mathrm{H} ; J$ values determined by Gaussian multiplication $(\mathrm{LB}=-3.0 \mathrm{~Hz}, \mathrm{~GB}=0.1 \mathrm{~Hz})$, C5- $\left.H_{\mathrm{ax}}\right), 4.98\left(\mathrm{dq}, J=10.7,7.1,1 \mathrm{H},-\mathrm{OCH}_{2} \mathrm{CH}_{3}\right), 4.89\left(\mathrm{dq}, J=10.7,7.1,1 \mathrm{H},-\mathrm{OCH}_{2} \mathrm{CH}_{3}\right), 4.67$ $\left(\mathrm{d}, J=11.7,1 \mathrm{H},-\mathrm{OCH}_{2} \mathrm{Ph}\right), 4.64\left(\mathrm{~d}, J=11.7,1 \mathrm{H},-\mathrm{OCH}_{2} \mathrm{Ph}\right), 4.28$ (sextet, $J=2.0,1 \mathrm{H} ; J$ value determined by Gaussian multiplication $\left.(\mathrm{LB}=-3.0 \mathrm{~Hz}, \mathrm{~GB}=0.09 \mathrm{~Hz}), \mathrm{C} 4-H_{\mathrm{eq}}\right), 3.30(\mathrm{ddd}, J=$ 20.7, 7.8, 2.6, $\left.1 \mathrm{H}, \mathrm{C} 2-H_{\mathrm{eq}}\right), 3.24$ (ddd, $\left.J=20.7,10.6,7.0,1 \mathrm{H}, \mathrm{C} 2-H_{\mathrm{ax}}\right), 2.46$ (dddd, $J=14.8$, 
7.0, 2.6, 2.0, 1H; $J$ values determined by Gaussian multiplication $(\mathrm{LB}=-4.0 \mathrm{~Hz}, \mathrm{~GB}=0.05 \mathrm{~Hz})$, C3- $H_{\mathrm{eq}}$ ), 2.33 (dddd, $J=14.8,10.6,7.8,2.0,1 \mathrm{H} ; J$ values determined by Gaussian multiplication $\left.(\mathrm{LB}=-4.0 \mathrm{~Hz}, \mathrm{~GB}=0.05 \mathrm{~Hz}), \mathrm{C} 3-H_{\mathrm{ax}}\right), 1.58\left(\mathrm{t}, J=7.1,3 \mathrm{H},-\mathrm{OCH}_{2} \mathrm{CH}_{3}\right) ;{ }^{13} \mathrm{C} \mathrm{NMR}(125 \mathrm{MHz}$, $\mathrm{CD}_{2} \mathrm{Cl}_{2}$, referenced to $\left.\delta 54.00\right) \delta 189.8,137.0,129.3,129.0,128.6,83.7,75.8,71.9,67.2,25.7$, 21.5, 13.9.

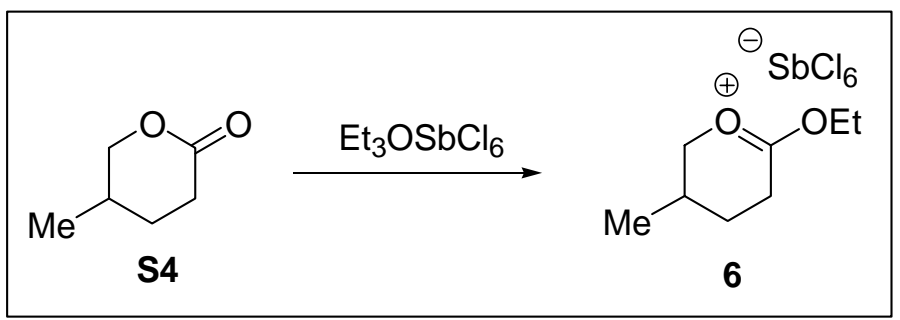

IIIB. 4-Methyl-1-ethoxy-2,3,4,5-tetrahydropyrylium hexachloroantimonate (6): To a 25-mL Schlenk flask containing a solution of freshly prepared 5-methyltetrahydropyran-2-one (S4) $(0.410 \mathrm{~g}, 3.58 \mathrm{mmol})$ in $12 \mathrm{~mL}$ of $\mathrm{CH}_{2} \mathrm{Cl}_{2}$ was added triethyloxonium hexachloroantimonate $(1.567 \mathrm{~g}, 3.580 \mathrm{mmol})$. The flask was protected from light, and the colorless mixture was stirred in a nitrogen atmosphere dry box for $6 \mathrm{~h}$. Anhydrous $\mathrm{Et}_{2} \mathrm{O}$ was added dropwise until the solution became translucent, but without the occurrence of precipitation. Then, the mixture was transferred to a $-20^{\circ} \mathrm{C}$ freezer to induce crystallization. After $18 \mathrm{~h}$ the supernatant was removed. The resulting solid was washed twice with $12 \mathrm{~mL}$ a 5:1 mixture of anhydrous $\mathrm{Et}_{2} \mathrm{O}: \mathrm{CH}_{2} \mathrm{Cl}_{2}$, and then was dried under vacuum to provide the crystalline product (colorless needles) ( $0.680 \mathrm{~g}$, 40\%): ${ }^{1} \mathrm{H}$ NMR (500 MHz, $\mathrm{CD}_{2} \mathrm{Cl}_{2}$, referenced to $\left.\delta 5.32\right) \delta 5.24(\mathrm{ddd}, J=11.2,5.0,2.0,1 \mathrm{H}$, $\left.\mathrm{C} 5-H_{\mathrm{eq}}\right), 4.94\left(\mathrm{dq}, J=10.7,7.1,1 \mathrm{H},-\mathrm{OCH}_{2} \mathrm{CH}_{3}\right), 4.90\left(\mathrm{dq}, J=10.7,7.1,1 \mathrm{H},-\mathrm{OCH}_{2} \mathrm{CH}_{3}\right), 4.77$ $\left(\mathrm{t}, J=11.2,1 \mathrm{H}, \mathrm{C} 5-H_{\text {ax }}\right), 3.30\left(\mathrm{ddd}, J=21.0,6.5,2.6,1 \mathrm{H}, \mathrm{C} 2-H_{\mathrm{eq}}\right), 3.18(\mathrm{ddd}, J=21.0,10.2$, 7.7, $\left.1 \mathrm{H}, \mathrm{C} 2-H_{\mathrm{ax}}\right), 2.44$ (ddqdd, $\left.J=14.1,11.2,6.7,6.2,5.0,1 \mathrm{H}, \mathrm{C} 4-H_{\mathrm{ax}}\right), 2.21$ (ddddd, $J=11.4$, $\left.7.7,6.2,2.6,2.0,1 \mathrm{H}, \mathrm{C} 3-H_{\mathrm{eq}}\right), 1.86$ (dddd, $\left.J=14.1,11.4,10.2,6.5,1 \mathrm{H}, \mathrm{C} 3-H_{\mathrm{ax}}\right), 1.59(\mathrm{t}, J=$ 7.1, 3H, $\left.-\mathrm{OCH}_{2} \mathrm{CH}_{3}\right), 1.14\left(\mathrm{~d}, J=6.7,3 \mathrm{H}, \mathrm{C} 4-\mathrm{CH}_{3}\right) ;{ }^{13} \mathrm{C} \mathrm{NMR}\left(125 \mathrm{MHz}, \mathrm{CD}_{2} \mathrm{Cl}_{2}\right.$, referenced 
to $\delta 54.00) \delta 189.7,86.2,75.6,29.4,27.6,24.4,15.5,14.0$; IR (KBr) 2966, 2901, 1694, 1564, 1461, $1384 \mathrm{~cm}^{-1}$. Anal. Calcd for $\mathrm{C}_{8} \mathrm{H}_{15} \mathrm{O}_{2} \mathrm{SbCl}_{6}: \mathrm{C}, 20.11 ; \mathrm{H}, 3.17$. Found: C, 19.92; H, 3.28 .

Note: Several attempts were made to study this compound using X-ray crystallography. Decomposition often occurred upon prolonged exposure of this cation to solvent under conditions designed to grow X-ray quality crystals (vapor diffusion of $\mathrm{Et}_{2} \mathrm{O}$ into a $\mathrm{CH}_{2} \mathrm{Cl}_{2}$ solution). Several crystals of the pure product were mounted on the diffractometer, but cracked upon exposure to the cold (ca. $-115^{\circ} \mathrm{C}$ ) stream of nitrogen gas. An X-ray structure was obtained for an identifiable decomposition product (9) derived from 6 . Structural data for this decomposition product and a rationale for its formation appear in section IVC.

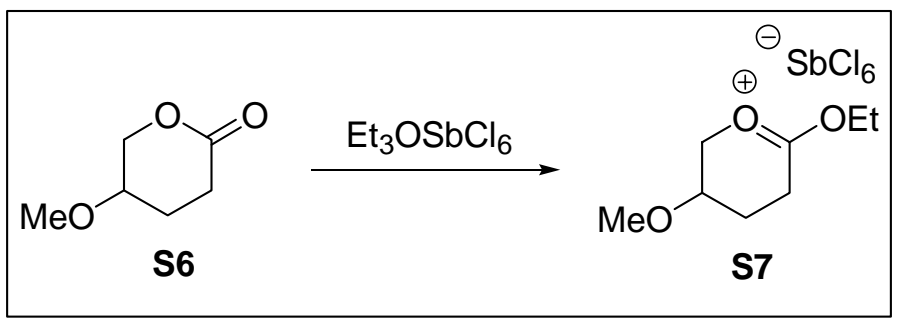

IIIC. 1-Ethoxy-4-methoxy-2,3,4,5-tetrahydropyrylium hexachloroantimonate (S7):

To a 10-mL Schlenk flask containing a solution of freshly prepared 5-methoxytetrahydropyran2-one (S6) $(0.130 \mathrm{~g}, 1.00 \mathrm{mmol})$ in $3.5 \mathrm{~mL}$ of $\mathrm{CH}_{2} \mathrm{Cl}_{2}$ was added triethyloxonium hexachloroantimonate $(0.438 \mathrm{~g}, 1.00 \mathrm{mmol})$. The flask was protected from light, and the colorless mixture was stirred at ambient temperature in a nitrogen atmosphere dry box for $3 \mathrm{~h}$. Anhydrous $\mathrm{Et}_{2} \mathrm{O}$ was added dropwise until the solution became translucent, but without the occurrence of precipitation. Then, the mixture was transferred to a $-20{ }^{\circ} \mathrm{C}$ freezer to induce crystallization. After $18 \mathrm{~h}$ the supernatant was removed. The resulting solid was washed twice with $3 \mathrm{~mL}$ a $5: 1$ mixture of anhydrous $\mathrm{Et}_{2} \mathrm{O}: \mathrm{CH}_{2} \mathrm{Cl}_{2}$, and then was dried under vacuum to provide the crystalline product (colorless needles) $(0.202 \mathrm{~g}, 41 \%):{ }^{1} \mathrm{H} \mathrm{NMR}\left(500 \mathrm{MHz}, \mathrm{CD}_{2} \mathrm{Cl}_{2}\right.$, referenced to $\delta 5.32) \delta 5.36\left(\mathrm{ddd}, J=12.3,2.0,1.4,1 \mathrm{H}, \mathrm{C} 5-H_{\text {eq }}\right), 5.29(\mathrm{dd}, J=12.3,2.0,1 \mathrm{H} \mathrm{C} 5-$ 
$\left.H_{\text {ax }}\right), 4.98\left(\mathrm{dq}, J=10.6,7.2,1 \mathrm{H},-\mathrm{OCH}_{2} \mathrm{CH}_{3}\right), 4.90\left(\mathrm{dq}, J=10.6,7.2,1 \mathrm{H},-\mathrm{OCH}_{2} \mathrm{CH}_{3}\right), 4.11$

(sextet, $J=2.0,1 \mathrm{H} ; J$ value determined by Gaussian multiplication $(\mathrm{LB}=-4.0 \mathrm{~Hz}, \mathrm{~GB}=0.2$ $\left.\mathrm{Hz}), \mathrm{C} 4-H_{\mathrm{eq}}\right), 3.45\left(\mathrm{~s}, 3 \mathrm{H},-\mathrm{OCH}_{3}\right), 3.27$ (ddt, $\left.J=20.5,7.0,1.4,1 \mathrm{H}, \mathrm{C} 2-H_{\mathrm{eq}}\right), 3.17$ (ddd, $J=$ $\left.20.5,11.0,7.4,1 \mathrm{H}, \mathrm{C} 2-H_{\mathrm{ax}}\right), 2.50$ (dddd, $\left.J=14.8,7.4,7.0,2.0,1 \mathrm{H}, \mathrm{C} 3-H_{\mathrm{eq}}\right), 2.35$ (dddd, $J=$

$\left.14.8,11.0,2.0,1.4,1 \mathrm{H}, \mathrm{C} 3-H_{\mathrm{ax}}\right), 1.60\left(\mathrm{t}, J=7.2,3 \mathrm{H},-\mathrm{OCH}_{2} \mathrm{CH}_{3}\right) ;{ }^{13} \mathrm{C}$ NMR $(125 \mathrm{MHz}$, $\mathrm{CD}_{2} \mathrm{Cl}_{2}$, referenced to $\left.\delta 54.00\right) \delta 189.9,83.1,75.6,69.2,57.2,25.5,21.0,14.0$.

\section{Proofs of Structure, Conformation, and Stereochemistry}

\section{IVA. Preferred Pseudoaxial Orientation of S3 is Independent of Solvent}

We sought to determine if the predominance of the pseudoaxial orientation of the benzyloxy substituent in $\mathbf{S 3}$ is dependent upon solvent and found that it is not. We could not accurately determine the position of the equilibrium between the equatorial and axial conformers. Available methods to do so employ a Karplus equation modified for electronegative substituents on cyclohexene, ${ }^{12}$ but the known conformational variability of substituted $\delta$-valerolactones precludes confident determination of the major ring conformation(s) present. The coalescence temperatures for these flexible rings are presumed to be lower than what can be determined using our instrumentation because the barrier for conformational inversion in six-membered rings containing an $\mathrm{sp}^{2}$-hybridized atom is low. Coupling constant values for the C-4 methine proton in C-4 alkoxysubstituted six-membered ring lactones can vary between approximately 2.1 and $9.7 \mathrm{~Hz} .{ }^{13}$ Lactone $\mathbf{S 3}$ was analyzed by ${ }^{1} \mathrm{H}$ NMR spectroscopy in a variety of deuterated solvents. The resulting coupling constant data for the characteristic C4-methine proton appears in the table below. We assumed that the $J$ values determined by Ghosh ${ }^{13}$ for the C4- 
methine proton in decalones $\mathbf{7}$ and $\mathbf{8}$ (below and main text) represented the upper and lower boundaries for coupling constants exhibited by this proton. Using the same weighted averaging method employed by Leblanc ${ }^{14}$ to determine the axial:equatorial population of 4-acetoxy- $\delta$-lactams (X-ray crystallography determined the major conformer to be axial), we can estimate the position of the equilibrium for $\mathbf{S 3}$.

We attempted to replicate this analysis to determine whether the observed pseudoaxial conformation of $\mathbf{5}$ was independent of solvent. Unfortunately, the salt is not soluble in $\mathrm{C}_{6} \mathrm{D}_{6}$, and it reacts with acetone- $d_{6}$ and methanol- $d_{4}$ to give products that no longer resemble $\mathbf{5}$. Since the spectrum of 5 in acetone- $d_{6}$ contains mostly starting lactone S3, cleavage of the ethyl group has most likely occurred in this solvent. The spectrum of 5 in methanol- $d_{4}$, however, most resembles the acyclic ester resulting from attack by the solvent at ring carbon C-5. Both of these decomposition pathways are known to occur

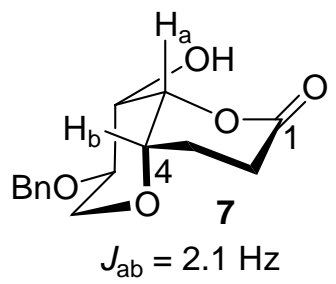

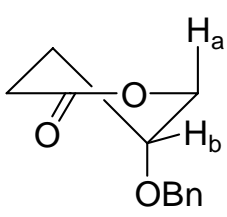

S3

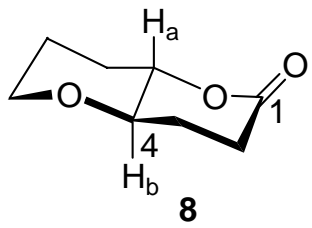

$J_{\mathrm{ab}}=9.7 \mathrm{~Hz}$

for lactonium ion salts of this type upon exposure to nucleophiles. ${ }^{10}$

\begin{tabular}{|c|c|c|c|}
\hline Solvent & $\delta$ C4-methine & $J_{\mathrm{ab}}$ value $(\mathrm{Hz})$ & $\begin{array}{c}\text { Estimated } \\
\text { axial:equatorial }\end{array}$ \\
\hline $\mathrm{C}_{6} \mathrm{D}_{6}$ & $2.89^{a}$ & 4.1 & $74: 26$ \\
\hline $\mathrm{CD}_{2} \mathrm{Cl}_{2}$ & $3.84^{b}$ & 3.0 & $88: 12$ \\
\hline $\mathrm{CDCl}_{3}$ & $3.89^{b}$ & 3.1 & $87: 13$ \\
\hline$\left(\mathrm{CD}_{3}\right)_{2} \mathrm{CO}$ & $3.94^{a}$ & 3.0 & $88: 12$ \\
\hline
\end{tabular}




\begin{tabular}{|c|c|c|c|}
\hline $\mathrm{CD}_{3} \mathrm{OD}$ & $3.89^{a}$ & 2.8 & $92: 8$ \\
\hline
\end{tabular}

${ }^{a}$ referenced to residual solvent peak

${ }^{b}$ referenced to TMS

${ }^{c}$ weighted average of observed $J$ value compared to $J_{\mathrm{ab}}=2.1 \mathrm{~Hz}$ in 7 and $9.7 \mathrm{~Hz}$ in 8

IVB. X-ray Crystallographic Data for 5 

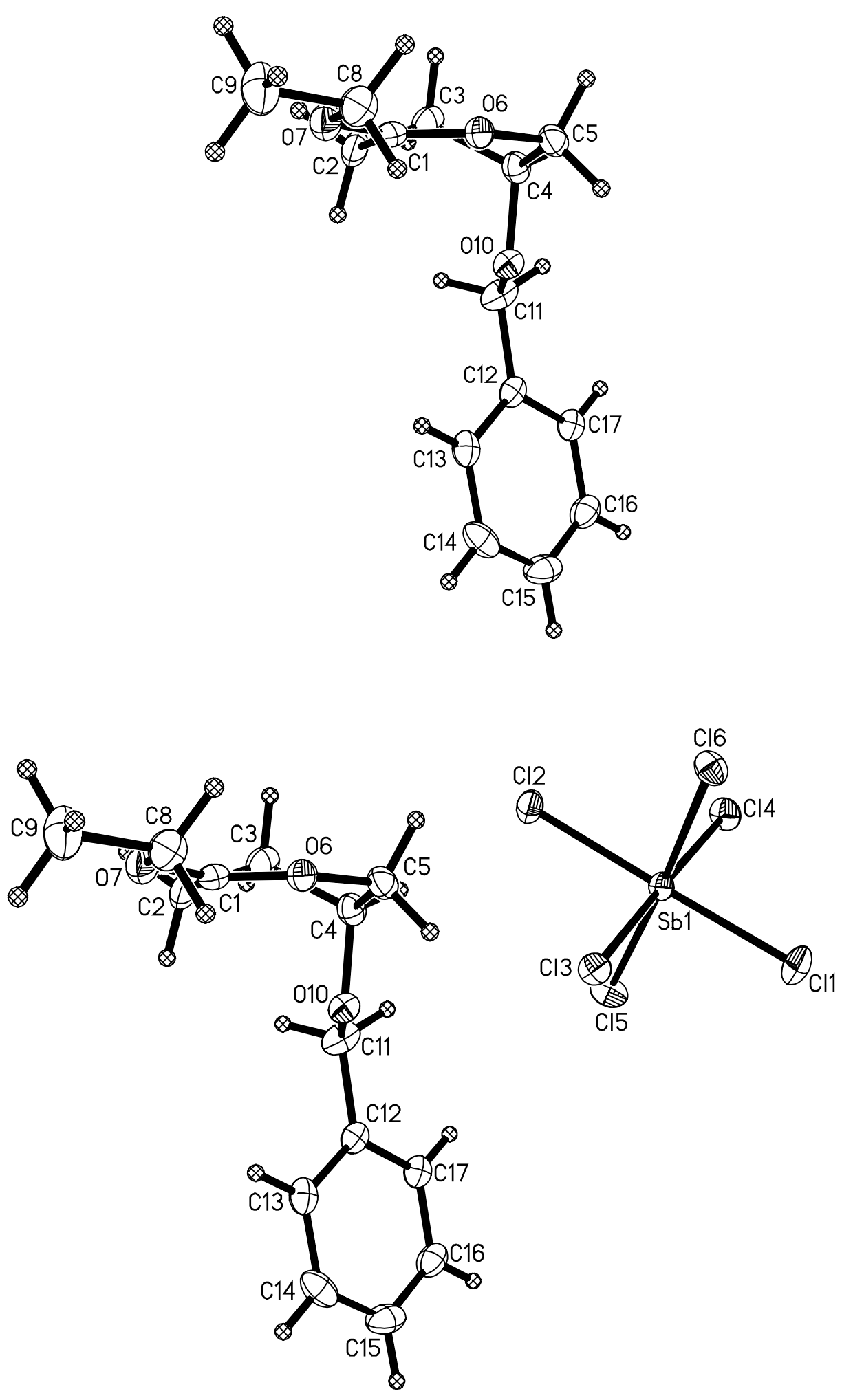

X-ray Data Collection, Structure Solution and Refinement for 5. 
A colorless crystal of approximate dimensions $0.07 \times 0.33 \times 0.45 \mathrm{~mm}$ was mounted on a glass fiber and transferred to a Bruker CCD platform diffractometer. The SMART ${ }^{1}$ program package was used to determine the unit-cell parameters and for data collection $(25 \mathrm{sec} /$ frame scan time for a sphere of diffraction data). The raw frame data was processed using $\mathrm{SAINT}^{2}$ and $\mathrm{SADABS}^{3}$ to yield the reflection data file. Subsequent calculations were carried out using the SHELXTL $^{4}$ program. The diffraction symmetry was $\mathrm{mmm}$ and the systematic absences were consistent with the orthorhombic space group Pbca which was later determined to be correct.

The structure was solved by direct methods and refined on $\mathrm{F}^{2}$ by full-matrix least-squares techniques. The analytical scattering factors ${ }^{5}$ for neutral atoms were used throughout the analysis. Hydrogen atoms were included using a riding model. At convergence, wR2 $=0.0847$ and $\mathrm{GOF}=1.084$ for 217 variables refined against 5201 data. As a comparison for refinement on $\mathrm{F}, \mathrm{R} 1=0.0306$ for those 4270 data with $\mathrm{I}>2.0 \sigma(\mathrm{I})$.

References.

1. SMART Software Users Guide, Version 5.1, Bruker Analytical X-Ray Systems, Inc.; Madison, WI 1999.

2. SAINT Software Users Guide, Version 6.0, Bruker Analytical X-Ray Systems, Inc.; Madison, WI 1999.

3. Sheldrick, G. M. SADABS, Version 2.05, Bruker Analytical X-Ray Systems, Inc.; Madison, WI 2001.

4. Sheldrick, G. M. SHELXTL Version 6.12, Bruker Analytical X-Ray Systems, Inc.; Madison, WI 2001.

5. International Tables for X-Ray Crystallography 1992, Vol. C., Dordrecht: Kluwer Academic Publishers.

Definitions:

$\mathrm{wR} 2=\left[\Sigma\left[\mathrm{w}\left(\mathrm{F}_{\mathrm{o}}^{2}-\mathrm{F}_{\mathrm{c}}^{2}\right)^{2}\right] / \Sigma\left[\mathrm{w}\left(\mathrm{F}_{\mathrm{o}}^{2}\right)^{2}\right]\right]^{1 / 2}$

$\mathrm{R} 1=\Sigma|| \mathrm{F}_{\mathrm{o}}|-| \mathrm{F}_{\mathrm{c}} \| / \Sigma\left|\mathrm{F}_{\mathrm{o}}\right|$

Goof $=\mathrm{S}=\left[\Sigma\left[\mathrm{w}\left(\mathrm{F}_{\mathrm{o}}^{2}-\mathrm{F}_{\mathrm{c}}{ }^{2}\right)^{2}\right] /(\mathrm{n}-\mathrm{p})\right]^{1 / 2}$ where $\mathrm{n}$ is the number of reflections and $\mathrm{p}$ is the total number of parameters refined.

The thermal ellipsoid plot is shown at the $30 \%$ probability level for clarity.

Table 1. Crystal data and structure refinement for 5 (kaw59). 
Identification code

Empirical formula

Formula weight

Temperature

Wavelength

Crystal system

Space group

Unit cell dimensions

Volume

$\mathrm{Z}$

Density (calculated)

Absorption coefficient

$\mathrm{F}(000)$

Crystal color

Crystal size

Theta range for data collection

Index ranges

Reflections collected

Independent reflections

Completeness to theta $=28.29^{\circ}$

Absorption correction

Max. and min. transmission

Refinement method

Data / restraints / parameters

Goodness-of-fit on $\mathrm{F}^{2}$ kaw59

$\mathrm{C}_{14} \mathrm{H}_{19} \mathrm{O}_{3} \mathrm{Cl}_{6} \mathrm{Sb}$

569.74

163(2) K

$0.71073 \AA$

Orthorhombic

Pbca

$\mathrm{a}=17.998(3) \AA \quad \alpha=90^{\circ}$.

$\mathrm{b}=13.063(2) \AA$

$\beta=90^{\circ}$.

$\mathrm{c}=18.084(3) \AA$

4251.7(11) $\AA^{3}$

8

$1.780 \mathrm{Mg} / \mathrm{m}^{3}$

$2.062 \mathrm{~mm}^{-1}$

2240

colorless

$0.45 \times 0.33 \times 0.07 \mathrm{~mm}^{3}$

2.23 to $28.29^{\circ}$.

$-23 \leq h \leq 23,-17 \leq k \leq 17,-23 \leq l \leq 23$

42057

$5201[\mathrm{R}(\mathrm{int})=0.0305]$

$98.5 \%$

Semi-empirical from equivalents

0.8691 and 0.4572

Full-matrix least-squares on $\mathrm{F}^{2}$

$5201 / 0 / 217$

1.084

Final $\mathrm{R}$ indices $[\mathrm{I}>2 \operatorname{sigma}(\mathrm{I})=4270$ data $] \mathrm{R} 1=0.0306, \mathrm{wR} 2=0.0743$

$\mathrm{R}$ indices (all data)

$\mathrm{R} 1=0.0437, \mathrm{wR} 2=0.0847$

Largest diff. peak and hole
1.353 and -0.677 e. $\AA^{-3}$ 
Table 2. Atomic coordinates ( $\left.\times 10^{4}\right)$ and equivalent isotropic displacement parameters $\left(\AA^{2} \mathrm{x}\right.$ $\left.10^{3}\right)$ for kaw59. $\mathrm{U}(\mathrm{eq})$ is defined as one third of the trace of the orthogonalized $\mathrm{U}^{\mathrm{ij}}$ tensor.

\begin{tabular}{lrrrr}
\hline & $\mathrm{x}$ & $\mathrm{y}$ & $\mathrm{z}$ & $\mathrm{U}(\mathrm{eq})$ \\
\hline $\mathrm{Sb}(1)$ & $11147(1)$ & $4391(1)$ & $2433(1)$ & $27(1)$ \\
$\mathrm{Cl}(1)$ & $12303(1)$ & $3634(1)$ & $2723(1)$ & $50(1)$ \\
$\mathrm{Cl}(2)$ & $9996(1)$ & $5165(1)$ & $2121(1)$ & $39(1)$ \\
$\mathrm{Cl}(3)$ & $10895(1)$ & $4597(1)$ & $3709(1)$ & $35(1)$ \\
$\mathrm{Cl}(4)$ & $11420(1)$ & $4137(1)$ & $1157(1)$ & $40(1)$ \\
$\mathrm{Cl}(5)$ & $10545(1)$ & $2783(1)$ & $2467(1)$ & $44(1)$ \\
$\mathrm{Cl}(6)$ & $11687(1)$ & $6033(1)$ & $2388(1)$ & $40(1)$ \\
$\mathrm{C}(1)$ & $8440(2)$ & $7039(2)$ & $4926(2)$ & $34(1)$ \\
$\mathrm{C}(2)$ & $7909(2)$ & $6373(3)$ & $4513(2)$ & $46(1)$ \\
$\mathrm{C}(3)$ & $8266(2)$ & $6002(3)$ & $3793(2)$ & $43(1)$ \\
$\mathrm{C}(4)$ & $9024(2)$ & $5576(3)$ & $3950(2)$ & $35(1)$ \\
$\mathrm{C}(5)$ & $9514(2)$ & $6365(3)$ & $4311(2)$ & $36(1)$ \\
$\mathrm{O}(6)$ & $9135(1)$ & $7025(2)$ & $4853(1)$ & $36(1)$ \\
$\mathrm{O}(7)$ & $8153(1)$ & $7671(2)$ & $5388(2)$ & $42(1)$ \\
$\mathrm{C}(8)$ & $8655(2)$ & $8341(3)$ & $5822(2)$ & $42(1)$ \\
$\mathrm{C}(9)$ & $8172(3)$ & $9001(4)$ & $6283(3)$ & $59(1)$ \\
$\mathrm{O}(10)$ & $8990(1)$ & $4722(2)$ & $4446(1)$ & $34(1)$ \\
$\mathrm{C}(11)$ & $8710(2)$ & $3823(3)$ & $4095(2)$ & $42(1)$ \\
$\mathrm{C}(12)$ & $8866(2)$ & $2936(2)$ & $4607(2)$ & $31(1)$ \\
$\mathrm{C}(13)$ & $8657(2)$ & $2968(3)$ & $5345(2)$ & $38(1)$ \\
$\mathrm{C}(14)$ & $8821(2)$ & $2158(3)$ & $5810(2)$ & $48(1)$ \\
$\mathrm{C}(15)$ & $9191(2)$ & $1308(3)$ & $5532(3)$ & $51(1)$ \\
$\mathrm{C}(16)$ & $9385(2)$ & $1267(3)$ & $4799(2)$ & $44(1)$ \\
$\mathrm{C}(17)$ & $9227(2)$ & $2078(3)$ & $4336(2)$ & $35(1)$ \\
& & & & \\
\hline
\end{tabular}


Table 3. Bond lengths $[\AA]$ and angles $\left[{ }^{\circ}\right]$ for kaw59.

\begin{tabular}{lc}
\hline $\mathrm{Sb}(1)-\mathrm{Cl}(6)$ & $2.3559(9)$ \\
$\mathrm{Sb}(1)-\mathrm{Cl}(1)$ & $2.3619(10)$ \\
$\mathrm{Sb}(1)-\mathrm{Cl}(5)$ & $2.3652(9)$ \\
$\mathrm{Sb}(1)-\mathrm{Cl}(3)$ & $2.3669(9)$ \\
$\mathrm{Sb}(1)-\mathrm{Cl}(2)$ & $2.3739(9)$ \\
$\mathrm{Sb}(1)-\mathrm{Cl}(4)$ & $2.3825(9)$ \\
$\mathrm{C}(1)-\mathrm{O}(6)$ & $1.257(4)$ \\
$\mathrm{C}(1)-\mathrm{O}(7)$ & $1.283(4)$ \\
$\mathrm{C}(1)-\mathrm{C}(2)$ & $1.493(5)$ \\
$\mathrm{C}(2)-\mathrm{C}(3)$ & $1.530(6)$ \\
$\mathrm{C}(3)-\mathrm{C}(4)$ & $1.500(5)$ \\
$\mathrm{C}(4)-\mathrm{O}(10)$ & $1.433(4)$ \\
$\mathrm{C}(4)-\mathrm{C}(5)$ & $1.506(5)$ \\
$\mathrm{C}(5)-\mathrm{O}(6)$ & $1.473(4)$ \\
$\mathrm{O}(7)-\mathrm{C}(8)$ & $1.482(4)$ \\
$\mathrm{C}(8)-\mathrm{C}(9)$ & $1.480(6)$ \\
$\mathrm{O}(10)-\mathrm{C}(11)$ & $1.428(4)$ \\
$\mathrm{C}(11)-\mathrm{C}(12)$ & $1.509(5)$ \\
$\mathrm{C}(12)-\mathrm{C}(17)$ & $1.384(5)$ \\
$\mathrm{C}(12)-\mathrm{C}(13)$ & $1.387(5)$ \\
$\mathrm{C}(13)-\mathrm{C}(14)$ & $1.384(6)$ \\
$\mathrm{C}(14)-\mathrm{C}(15)$ & $1.390(6)$ \\
$\mathrm{C}(15)-\mathrm{C}(16)$ & $1.372(6)$ \\
$\mathrm{C}(16)-\mathrm{C}(17)$ & $1.380(5)$ \\
$\mathrm{Cl}(6)-\mathrm{Sb}(1)-\mathrm{Cl}(1)$ & $91.49(4)$ \\
$\mathrm{Cl}(6)-\mathrm{Sb}(1)-\mathrm{Cl}(5)$ & $177.02(4)$ \\
$\mathrm{Cl}(1)-\mathrm{Sb}(1)-\mathrm{Cl}(5)$ & $91.49(4)$ \\
$\mathrm{Cl}(6)-\mathrm{Sb}(1)-\mathrm{Cl}(3)$ & $90.53(3)$ \\
$\mathrm{Cl}(1)-\mathrm{Sb}(1)-\mathrm{Cl}(3)$ & $89.99(3)$ \\
$\mathrm{Cl}(5)-\mathrm{Sb}(1)-\mathrm{Cl}(3)$ & $89.30(3)$ \\
$\mathrm{Cl}(6)-\mathrm{Sb}(1)-\mathrm{Cl}(2)$ & $87.92(4)$ \\
$\mathrm{Cl}(1)-\mathrm{Sb}(1)-\mathrm{Cl}(2)$ & $178.92(4)$ \\
$\mathrm{Cl}(5)-\mathrm{Sb}(1)-\mathrm{Cl}(2)$ & $89.11(4)$ \\
& \\
& \\
&
\end{tabular}




$\begin{array}{lr}\mathrm{Cl}(3)-\mathrm{Sb}(1)-\mathrm{Cl}(2) & 90.92(3) \\ \mathrm{Cl}(6)-\mathrm{Sb}(1)-\mathrm{Cl}(4) & 90.49(3) \\ \mathrm{Cl}(1)-\mathrm{Sb}(1)-\mathrm{Cl}(4) & 88.60(4) \\ \mathrm{Cl}(5)-\mathrm{Sb}(1)-\mathrm{Cl}(4) & 89.76(3) \\ \mathrm{Cl}(3)-\mathrm{Sb}(1)-\mathrm{Cl}(4) & 178.28(3) \\ \mathrm{Cl}(2)-\mathrm{Sb}(1)-\mathrm{Cl}(4) & 90.50(3) \\ \mathrm{O}(6)-\mathrm{C}(1)-\mathrm{O}(7) & 118.6(3) \\ \mathrm{O}(6)-\mathrm{C}(1)-\mathrm{C}(2) & 125.1(3) \\ \mathrm{O}(7)-\mathrm{C}(1)-\mathrm{C}(2) & 116.3(3) \\ \mathrm{C}(1)-\mathrm{C}(2)-\mathrm{C}(3) & 110.0(3) \\ \mathrm{C}(4)-\mathrm{C}(3)-\mathrm{C}(2) & 109.8(3) \\ \mathrm{O}(10)-\mathrm{C}(4)-\mathrm{C}(3) & 111.7(3) \\ \mathrm{O}(10)-\mathrm{C}(4)-\mathrm{C}(5) & 106.6(3) \\ \mathrm{C}(3)-\mathrm{C}(4)-\mathrm{C}(5) & 111.2(3) \\ \mathrm{O}(6)-\mathrm{C}(5)-\mathrm{C}(4) & 114.6(3) \\ \mathrm{C}(1)-\mathrm{O}(6)-\mathrm{C}(5) & 122.7(3) \\ \mathrm{C}(1)-\mathrm{O}(7)-\mathrm{C}(8) & 118.7(3) \\ \mathrm{C}(9)-\mathrm{C}(8)-\mathrm{O}(7) & 106.6(3) \\ \mathrm{C}(11)-\mathrm{O}(10)-\mathrm{C}(4) & 112.1(3) \\ \mathrm{O}(10)-\mathrm{C}(11)-\mathrm{C}(12) & 107.0(3) \\ \mathrm{C}(17)-\mathrm{C}(12)-\mathrm{C}(13) & 119.5(3) \\ \mathrm{C}(17)-\mathrm{C}(12)-\mathrm{C}(11) & 119.4(3) \\ \mathrm{C}(13)-\mathrm{C}(12)-\mathrm{C}(11) & 121.1(3) \\ \mathrm{C}(14)-\mathrm{C}(13)-\mathrm{C}(12) & 120.3(3) \\ \mathrm{C}(13)-\mathrm{C}(14)-\mathrm{C}(15) & 119.6(4) \\ \mathrm{C}(16)-\mathrm{C}(15)-\mathrm{C}(14) & 120.1(4) \\ \mathrm{C}(15)-\mathrm{C}(16)-\mathrm{C}(17) & 120.3(4) \\ \mathrm{C}(16)-\mathrm{C}(17)-\mathrm{C}(12) & 120.3(4) \\ & \end{array}$


Table 4. Anisotropic displacement parameters $\left(\AA^{2} \times 10^{3}\right)$ for kaw59. The anisotropic displacement factor exponent takes the form: $-2 \pi^{2}\left[h^{2} a^{* 2} U^{11}+\ldots+2 h \mathrm{k} \mathrm{a}^{*} \mathrm{~b}^{*} \mathrm{U}^{12}\right]$

\begin{tabular}{lcccccc}
\hline & $\mathrm{U}^{11}$ & $\mathrm{U}^{22}$ & $\mathrm{U}^{33}$ & $\mathrm{U}^{23}$ & $\mathrm{U}^{13}$ & $\mathrm{U}^{12}$ \\
\hline $\mathrm{Sb}(1)$ & $27(1)$ & $29(1)$ & $25(1)$ & $-5(1)$ & $2(1)$ & $2(1)$ \\
$\mathrm{Cl}(1)$ & $37(1)$ & $56(1)$ & $56(1)$ & $-5(1)$ & $-4(1)$ & $18(1)$ \\
$\mathrm{Cl}(2)$ & $31(1)$ & $54(1)$ & $31(1)$ & $-1(1)$ & $0(1)$ & $10(1)$ \\
$\mathrm{Cl}(3)$ & $43(1)$ & $39(1)$ & $24(1)$ & $-5(1)$ & $1(1)$ & $4(1)$ \\
$\mathrm{Cl}(4)$ & $48(1)$ & $45(1)$ & $28(1)$ & $-8(1)$ & $10(1)$ & $4(1)$ \\
$\mathrm{Cl}(5)$ & $60(1)$ & $36(1)$ & $36(1)$ & $-5(1)$ & $0(1)$ & $-14(1)$ \\
$\mathrm{Cl}(6)$ & $42(1)$ & $36(1)$ & $43(1)$ & $-5(1)$ & $5(1)$ & $-8(1)$ \\
$\mathrm{C}(1)$ & $39(2)$ & $25(2)$ & $37(2)$ & $8(1)$ & $5(1)$ & $5(1)$ \\
$\mathrm{C}(2)$ & $26(2)$ & $39(2)$ & $73(3)$ & $4(2)$ & $4(2)$ & $6(2)$ \\
$\mathrm{C}(3)$ & $32(2)$ & $47(2)$ & $50(2)$ & $2(2)$ & $-13(2)$ & $1(2)$ \\
$\mathrm{C}(4)$ & $29(2)$ & $44(2)$ & $31(2)$ & $-1(1)$ & $-1(1)$ & $-2(1)$ \\
$\mathrm{C}(5)$ & $30(2)$ & $42(2)$ & $36(2)$ & $4(2)$ & $1(1)$ & $-6(2)$ \\
$\mathrm{O}(6)$ & $26(1)$ & $38(1)$ & $43(1)$ & $7(1)$ & $-1(1)$ & $-1(1)$ \\
$\mathrm{O}(7)$ & $32(1)$ & $40(1)$ & $54(2)$ & $1(1)$ & $4(1)$ & $1(1)$ \\
$\mathrm{C}(8)$ & $37(2)$ & $46(2)$ & $43(2)$ & $-3(2)$ & $-7(2)$ & $-2(2)$ \\
$\mathrm{C}(9)$ & $45(2)$ & $56(3)$ & $75(3)$ & $-16(2)$ & $11(2)$ & $-1(2)$ \\
$\mathrm{O}(10)$ & $35(1)$ & $31(1)$ & $36(1)$ & $-2(1)$ & $-4(1)$ & $1(1)$ \\
$\mathrm{C}(11)$ & $47(2)$ & $32(2)$ & $48(2)$ & $-5(2)$ & $-22(2)$ & $-1(2)$ \\
$\mathrm{C}(12)$ & $24(2)$ & $29(2)$ & $39(2)$ & $-7(1)$ & $-9(1)$ & $-4(1)$ \\
$\mathrm{C}(13)$ & $29(2)$ & $36(2)$ & $47(2)$ & $-11(2)$ & $1(1)$ & $-5(1)$ \\
$\mathrm{C}(14)$ & $49(2)$ & $54(2)$ & $41(2)$ & $-1(2)$ & $5(2)$ & $-23(2)$ \\
$\mathrm{C}(15)$ & $53(2)$ & $38(2)$ & $63(3)$ & $15(2)$ & $-14(2)$ & $-10(2)$ \\
$\mathrm{C}(16)$ & $38(2)$ & $32(2)$ & $61(2)$ & $-2(2)$ & $-5(2)$ & $3(2)$ \\
$\mathrm{C}(17)$ & $31(2)$ & $33(2)$ & $41(2)$ & $-7(1)$ & $-2(1)$ & $-2(1)$ \\
& & & & & & \\
\hline
\end{tabular}


Table 5. Hydrogen coordinates ( $\left.\times 10^{4}\right)$ and isotropic displacement parameters $\left(\AA^{2} \times 10^{3}\right)$ for kaw59.

\begin{tabular}{|c|c|c|c|c|}
\hline & $\mathrm{x}$ & $\mathrm{y}$ & $\mathrm{z}$ & $\mathrm{U}(\mathrm{eq})$ \\
\hline $\mathrm{H}(2 \mathrm{~A})$ & 7452 & 6763 & 4399 & 55 \\
\hline $\mathrm{H}(2 \mathrm{~B})$ & 7771 & 5778 & 4822 & 55 \\
\hline $\mathrm{H}(3 \mathrm{~A})$ & 7951 & 5466 & 3566 & 52 \\
\hline $\mathrm{H}(3 \mathrm{~B})$ & 8306 & 6578 & 3440 & 52 \\
\hline $\mathrm{H}(4 \mathrm{~A})$ & 9257 & 5351 & 3475 & 42 \\
\hline $\mathrm{H}(5 \mathrm{~A})$ & 9929 & 6008 & 4562 & 43 \\
\hline $\mathrm{H}(5 \mathrm{~B})$ & 9732 & 6804 & 3920 & 43 \\
\hline $\mathrm{H}(8 \mathrm{~A})$ & 8987 & 7924 & 6138 & 50 \\
\hline $\mathrm{H}(8 \mathrm{~B})$ & 8964 & 8764 & 5488 & 50 \\
\hline $\mathrm{H}(9 \mathrm{~A})$ & 8482 & 9460 & 6582 & 88 \\
\hline $\mathrm{H}(9 \mathrm{~B})$ & 7869 & 8572 & 6610 & 88 \\
\hline $\mathrm{H}(9 \mathrm{C})$ & 7847 & 9407 & 5963 & 88 \\
\hline $\mathrm{H}(11 \mathrm{~A})$ & 8959 & 3715 & 3613 & 51 \\
\hline $\mathrm{H}(11 \mathrm{~B})$ & 8169 & 3888 & 4008 & 51 \\
\hline $\mathrm{H}(13 \mathrm{~A})$ & 8399 & 3548 & 5531 & 45 \\
\hline $\mathrm{H}(14 \mathrm{~A})$ & 8682 & 2184 & 6317 & 58 \\
\hline$H(15 A)$ & 9310 & 753 & 5850 & 62 \\
\hline $\mathrm{H}(16 \mathrm{~A})$ & 9629 & 679 & 4609 & 52 \\
\hline $\mathrm{H}(17 \mathrm{~A})$ & 9366 & 2048 & 3830 & 42 \\
\hline
\end{tabular}


Table 6. Torsion angles $\left[{ }^{\circ}\right]$ for kaw59.

\begin{tabular}{lc}
\hline $\mathrm{O}(6)-\mathrm{C}(1)-\mathrm{C}(2)-\mathrm{C}(3)$ & $-22.9(5)$ \\
$\mathrm{O}(7)-\mathrm{C}(1)-\mathrm{C}(2)-\mathrm{C}(3)$ & $157.0(3)$ \\
$\mathrm{C}(1)-\mathrm{C}(2)-\mathrm{C}(3)-\mathrm{C}(4)$ & $49.3(4)$ \\
$\mathrm{C}(2)-\mathrm{C}(3)-\mathrm{C}(4)-\mathrm{O}(10)$ & $60.7(4)$ \\
$\mathrm{C}(2)-\mathrm{C}(3)-\mathrm{C}(4)-\mathrm{C}(5)$ & $-58.2(4)$ \\
$\mathrm{O}(10)-\mathrm{C}(4)-\mathrm{C}(5)-\mathrm{O}(6)$ & $-83.4(3)$ \\
$\mathrm{C}(3)-\mathrm{C}(4)-\mathrm{C}(5)-\mathrm{O}(6)$ & $38.5(4)$ \\
$\mathrm{O}(7)-\mathrm{C}(1)-\mathrm{O}(6)-\mathrm{C}(5)$ & $-176.9(3)$ \\
$\mathrm{C}(2)-\mathrm{C}(1)-\mathrm{O}(6)-\mathrm{C}(5)$ & $3.0(5)$ \\
$\mathrm{C}(4)-\mathrm{C}(5)-\mathrm{O}(6)-\mathrm{C}(1)$ & $-10.6(4)$ \\
$\mathrm{O}(6)-\mathrm{C}(1)-\mathrm{O}(7)-\mathrm{C}(8)$ & $-1.0(5)$ \\
$\mathrm{C}(2)-\mathrm{C}(1)-\mathrm{O}(7)-\mathrm{C}(8)$ & $179.1(3)$ \\
$\mathrm{C}(1)-\mathrm{O}(7)-\mathrm{C}(8)-\mathrm{C}(9)$ & $178.2(3)$ \\
$\mathrm{C}(3)-\mathrm{C}(4)-\mathrm{O}(10)-\mathrm{C}(11)$ & $73.8(4)$ \\
$\mathrm{C}(5)-\mathrm{C}(4)-\mathrm{O}(10)-\mathrm{C}(11)$ & $-164.5(3)$ \\
$\mathrm{C}(4)-\mathrm{O}(10)-\mathrm{C}(11)-\mathrm{C}(12)$ & $167.1(3)$ \\
$\mathrm{O}(10)-\mathrm{C}(11)-\mathrm{C}(12)-\mathrm{C}(17)$ & $-128.1(3)$ \\
$\mathrm{O}(10)-\mathrm{C}(11)-\mathrm{C}(12)-\mathrm{C}(13)$ & $51.7(4)$ \\
$\mathrm{C}(17)-\mathrm{C}(12)-\mathrm{C}(13)-\mathrm{C}(14)$ & $1.4(5)$ \\
$\mathrm{C}(11)-\mathrm{C}(12)-\mathrm{C}(13)-\mathrm{C}(14)$ & $-178.4(3)$ \\
$\mathrm{C}(12)-\mathrm{C}(13)-\mathrm{C}(14)-\mathrm{C}(15)$ & $-0.6(5)$ \\
$\mathrm{C}(13)-\mathrm{C}(14)-\mathrm{C}(15)-\mathrm{C}(16)$ & $-0.7(6)$ \\
$\mathrm{C}(14)-\mathrm{C}(15)-\mathrm{C}(16)-\mathrm{C}(17)$ & $1.2(6)$ \\
$\mathrm{C}(15)-\mathrm{C}(16)-\mathrm{C}(17)-\mathrm{C}(12)$ & $-0.5(6)$ \\
$\mathrm{C}(13)-\mathrm{C}(12)-\mathrm{C}(17)-\mathrm{C}(16)$ & $-0.8(5)$ \\
$\mathrm{C}(11)-\mathrm{C}(12)-\mathrm{C}(17)-\mathrm{C}(16)$ & $179.0(3)$ \\
& \\
\hline
\end{tabular}




\section{IVC. X-ray Crystallographic Data for 9}

Although several attempts to study cation 6 using X-ray crystallography were unsuccessful, we were able to prove the structure of a decomposition product. Dioxocarbenium ion 9 likely resulted from decomposition of $\mathbf{6}$ which took place during the time required to grow $\mathrm{X}$-ray quality crystals. Normally, the alkylation of $\mathbf{S 4}$ by $\mathrm{Et}_{3} \mathrm{OSbCl}_{6}$ went to completion. Over time and in solution, however, the possibility exists that a dissociated chloride ligand from the $\mathrm{SbCl}_{6}^{-}$counterion could cleave the ethyl group from $\mathbf{6}$ to regenerate lactone $\mathbf{S 4}$. Mechanistically, decomposition product 9 most likely derives from nucleophilic attack of $\mathbf{6}$ at C5 by the starting material, lactone $\mathbf{S 4}$. The vulnerability of C-5 to attack by nucleophiles such as iodide ion has been observed in similar lactonium ion salts. ${ }^{10,11}$ This phenomenon supports the above rationale describing the formation of dioxocarbenium ion salt $\mathbf{9}$. Compound $\mathbf{9}$ was never observed in preparations of pure dioxocarbenium ion salt $\mathbf{6}$ and, hence, no additional characterization data can be provided. The structure of this new dioxocarbenium ion, 9, was proven by X-ray crystallography to contain essential elements of cation 6 and a straight-chain aliphatic ester. A pictorial description of this mechanism appears in the scheme below. We assert that the geometry of the six-membered ring in $\mathbf{9}$ is similar to $\mathbf{6}$ and also possesses a pseudoequatorial methyl substituent at C-4. In conjunction with this crystallographic data, the spectroscopic data obtained for cation $\mathbf{6}$, and the correlative data for C-5 methyl analogue $\mathbf{1 0},{ }^{10}$ we are confident that the C-4 methyl substituent in dioxocarbenium ion $\mathbf{6}$ is pseudoequatorial.

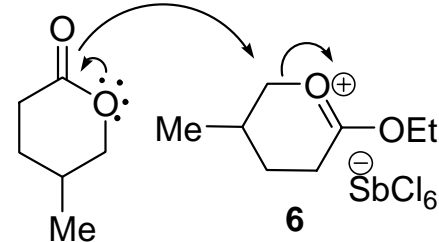

S4

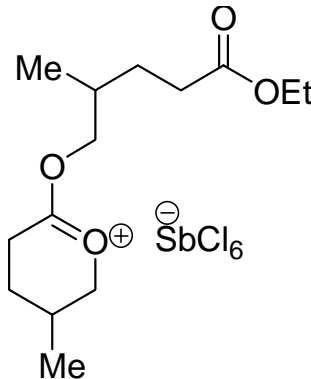

9 
X-ray data for 9 appears below, and is a disordered, but better refined composite structure. The picture of the composite structure is shown first, followed by clearer views of dioxocarbenium ion 9.
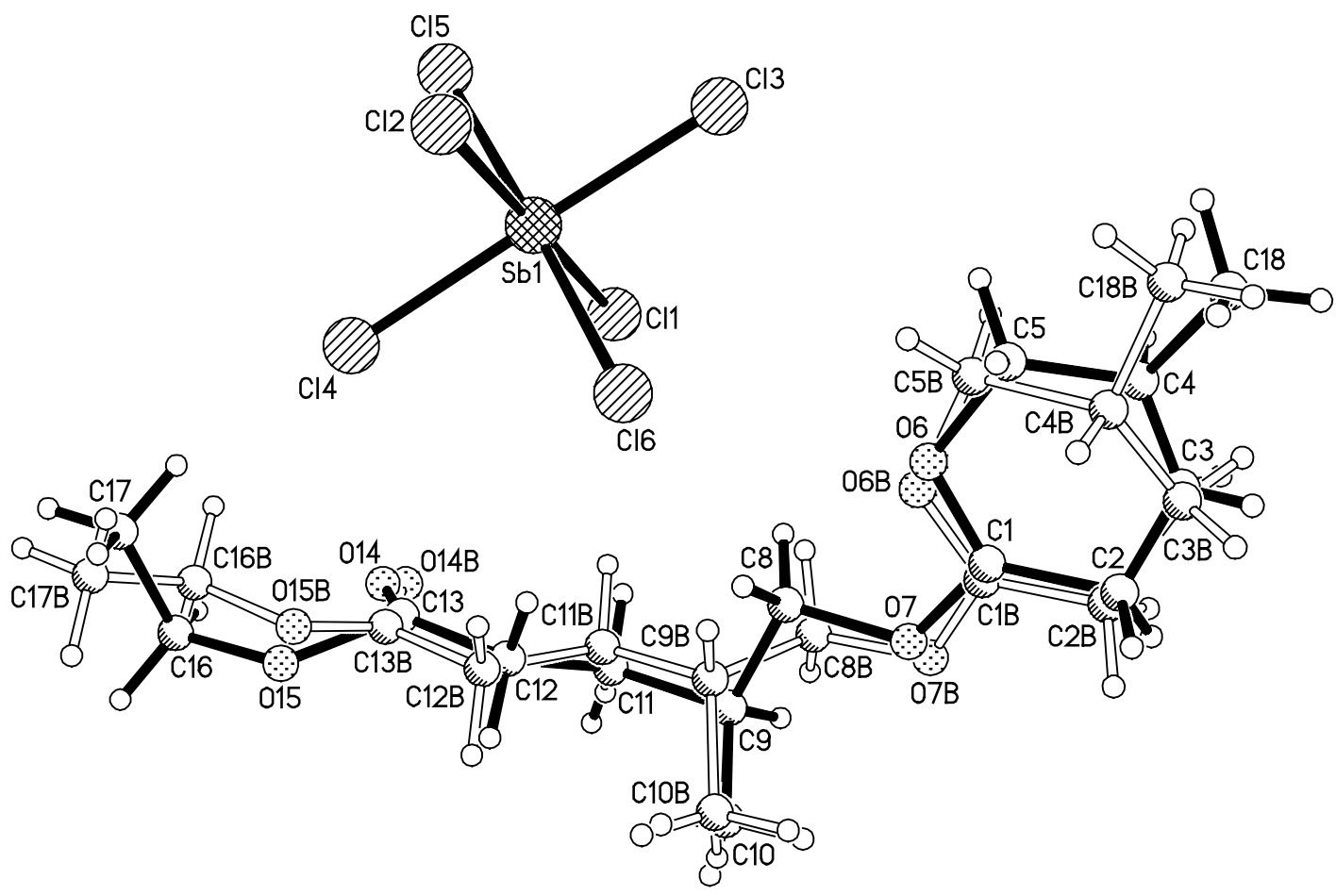


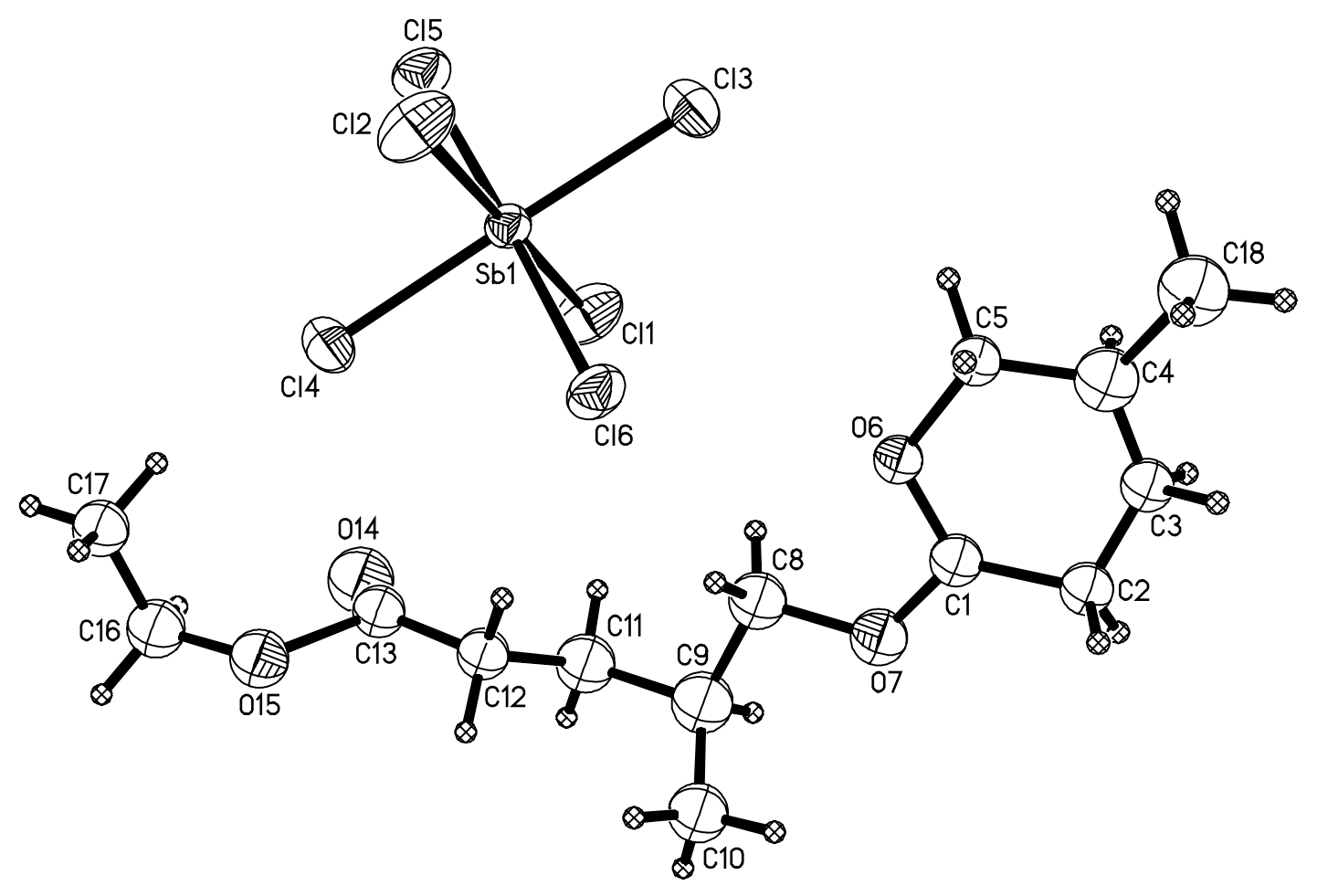




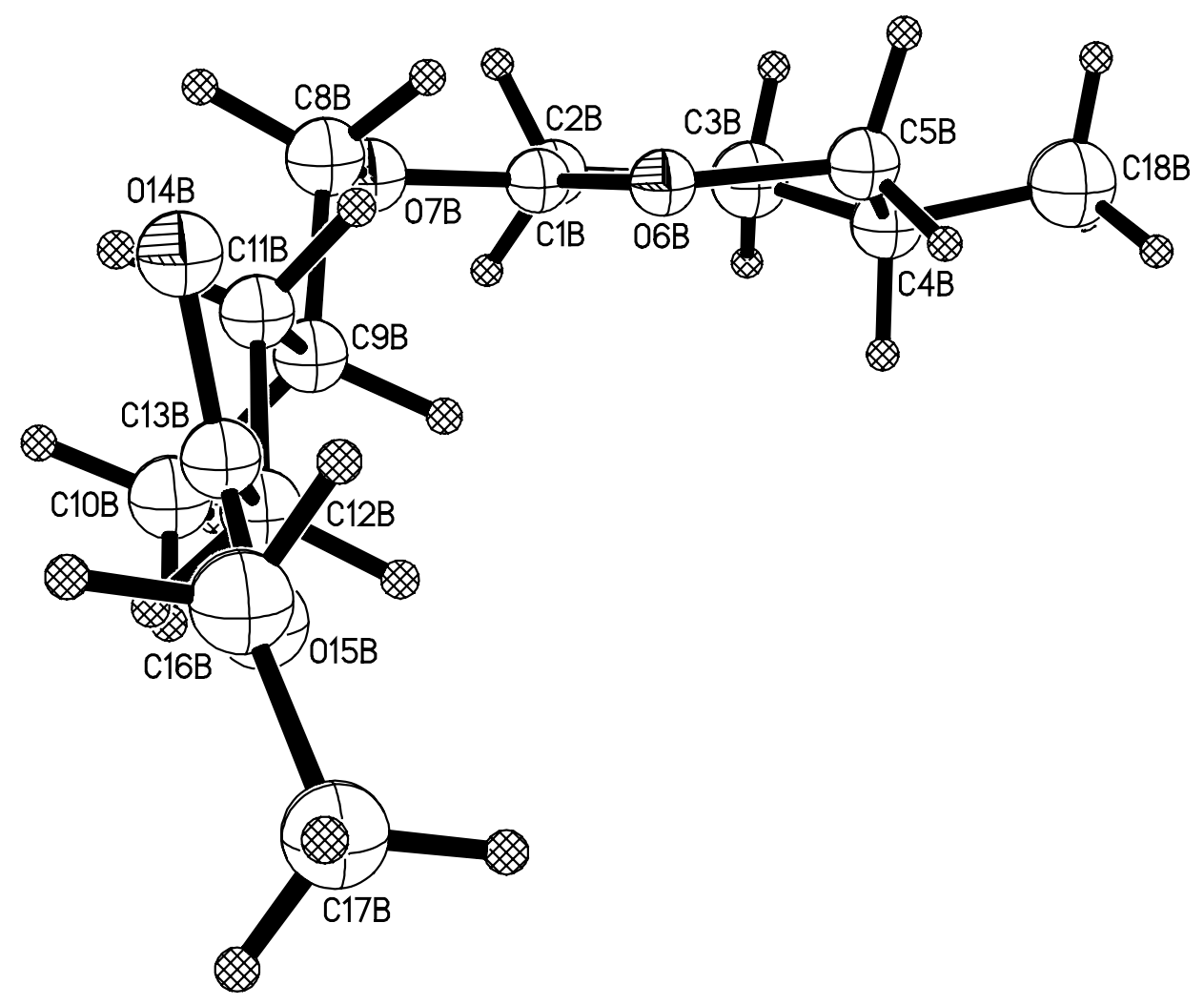

X-ray Data Collection, Structure Solution and Refinement for 9 (kaw64).

A colorless crystal of approximate dimensions $0.22 \times 0.31 \times 0.33 \mathrm{~mm}$ was mounted on a glass fiber and transferred to a Bruker CCD platform diffractometer. The SMART ${ }^{1}$ program package was used to determine the unit-cell parameters and for data collection $(25 \mathrm{sec} /$ frame scan time for a sphere of diffraction data). The raw frame data was processed using SAINT $^{2}$ and $\mathrm{SADABS}^{3}$ to yield the reflection data file. Subsequent calculations were carried out using the SHELXTL $^{4}$ program. The diffraction symmetry was $2 / m$ and the systematic absences were consistent with the centrosymmetric monoclinic space group $P 2_{1} / n$ which was later determined to be correct.

The structure was solved by direct methods and refined on $\mathrm{F}^{2}$ by full-matrix least-squares techniques. The analytical scattering factors ${ }^{5}$ for neutral atoms were used throughout the analysis. Hydrogen atoms were included using a riding model. Anisotropic refinement did not converge well. Several light atoms exhibited elongated ellipsoids and higher than acceptable thermal parameters. The most satisfactory refinement required the carbon, oxygen and hydrogen atoms be treated as disordered and included using multiple components with partial siteoccupancy-factors. At convergence, $w R 2=0.1117$ and Goof $=1.036$ for 209 variables refined 
against 4712 data $(0.80 \AA)$. As a comparison for refinement on $F, R 1=0.0396$ for those 3808 data with $\mathrm{I}>2.0 \sigma(\mathrm{I})$.

References.

6. SMART Software Users Guide, Version 5.1, Bruker Analytical X-Ray Systems, Inc.; Madison, WI 1999.

7. SAINT Software Users Guide, Version 6.0, Bruker Analytical X-Ray Systems, Inc.; Madison, WI 1999.

8. Sheldrick, G. M. SADABS, Version 2.05, Bruker Analytical X-Ray Systems, Inc.; Madison, WI 2001.

9. Sheldrick, G. M. SHELXTL Version 6.12, Bruker Analytical X-Ray Systems, Inc.; Madison, WI 2001.

10. International Tables for X-Ray Crystallography 1992, Vol. C., Dordrecht: Kluwer AcademicPublishers.

Definitions:

$$
\begin{aligned}
& \mathrm{wR} 2=\left[\Sigma\left[\mathrm{w}\left(\mathrm{F}_{\mathrm{o}}^{2}-\mathrm{F}_{\mathrm{c}}{ }^{2}\right)^{2}\right] / \Sigma\left[\mathrm{w}\left(\mathrm{F}_{\mathrm{o}}^{2}\right)^{2}\right]\right]^{1 / 2} \\
& \mathrm{R} 1=\Sigma\left\|\mathrm{F}_{\mathrm{o}}|-| \mathrm{F}_{\mathrm{c}}\right\| / \Sigma\left|\mathrm{F}_{\mathrm{o}}\right|
\end{aligned}
$$

Goof $=\mathrm{S}=\left[\Sigma\left[\mathrm{w}\left(\mathrm{F}_{\mathrm{o}}^{2}-\mathrm{F}_{\mathrm{c}}{ }^{2}\right)^{2}\right] /(\mathrm{n}-\mathrm{p})\right]^{1 / 2}$ where $\mathrm{n}$ is the number of reflections and $\mathrm{p}$ is the total number of parameters refined.

The thermal ellipsoid plot is shown at the $30 \%$ probability level for clarity. 
Table 1. Crystal data and structure refinement for 9 (kaw64).

Identification code

Empirical formula

Formula weight

Temperature

Wavelength

Crystal system

Space group

Unit cell dimensions

Volume

$\mathrm{Z}$

Density (calculated)

Absorption coefficient

$\mathrm{F}(000)$

Crystal color

Crystal size

Theta range for data collection

Index ranges

Reflections collected

Independent reflections

Completeness to theta $=26.37^{\circ}$

Absorption correction

Max. and min. transmission

Refinement method

Data / restraints / parameters

Goodness-of-fit on $\mathrm{F}^{2}$ kaw64

$\mathrm{C}_{14} \mathrm{H}_{25} \mathrm{O}_{4} \mathrm{Cl}_{6} \mathrm{Sb}$

591.79

208(2) K

$0.71073 \AA$

Monoclinic

$P 2{ }_{1} / n$

$\mathrm{a}=10.4080(19) \AA \quad \alpha=90^{\circ}$.

$\mathrm{b}=12.304(2) \AA$

$\beta=97.015(3)^{\circ}$.

$\mathrm{c}=18.297(3) \AA$

2325.5(7) $\AA^{3}$

4

$1.690 \mathrm{Mg} / \mathrm{m}^{3}$

$1.891 \mathrm{~mm}^{-1}$

1176

colorless

$0.33 \times 0.31 \times 0.22 \mathrm{~mm}^{3}$

2.00 to $26.37^{\circ}$.

$-13 \leq h \leq 12,-15 \leq k \leq 15,-22 \leq l \leq 22$

21538

$4712[\mathrm{R}(\mathrm{int})=0.0258]$

$99.1 \%$

Semi-empirical from equivalents

0.6811 and 0.5742

Full-matrix least-squares on $\mathrm{F}^{2}$

4712 / 0 / 209

1.036

Final $\mathrm{R}$ indices $[\mathrm{I}>2 \operatorname{sigma}(\mathrm{I})=3808$ data $] \mathrm{R} 1=0.0396, \mathrm{wR} 2=0.1027$

$\mathrm{R}$ indices (all data)

$\mathrm{R} 1=0.0512, \mathrm{wR} 2=0.1117$

Largest diff. peak and hole
1.036 and -0.563 e. $\AA^{-3}$ 
Table 2. Atomic coordinates ( $\left.\times 10^{4}\right)$ and equivalent isotropic displacement parameters $\left(\AA^{2} \mathrm{x}\right.$ $\left.10^{3}\right)$ for kaw64. $\mathrm{U}(\mathrm{eq})$ is defined as one third of the trace of the orthogonalized $\mathrm{U}^{\mathrm{ij}}$ tensor.

\begin{tabular}{lccll}
\hline & $\mathrm{x}$ & $\mathrm{y}$ & $\mathrm{z}$ & $\mathrm{U}(\mathrm{eq})$ \\
\hline $\mathrm{Sb}(1)$ & & & & \\
$\mathrm{Cl}(1)$ & $6018(1)$ & $7419(1)$ & $8633(1)$ & $43(1)$ \\
$\mathrm{Cl}(2)$ & $4595(2)$ & $6546(1)$ & $7722(1)$ & $85(1)$ \\
$\mathrm{Cl}(3)$ & $7433(2)$ & $8299(1)$ & $9539(1)$ & $85(1)$ \\
$\mathrm{Cl}(4)$ & $7687(2)$ & $6255(1)$ & $8358(1)$ & $89(1)$ \\
$\mathrm{Cl}(5)$ & $4305(2)$ & $8567(1)$ & $8897(1)$ & $81(1)$ \\
$\mathrm{Cl}(6)$ & $6568(1)$ & $8704(1)$ & $7764(1)$ & $66(1)$ \\
$\mathrm{C}(1)$ & $5443(1)$ & $6165(1)$ & $9524(1)$ & $65(1)$ \\
$\mathrm{C}(2)$ & $4358(17)$ & $2668(13)$ & $8777(9)$ & $58(4)$ \\
$\mathrm{C}(3)$ & $4719(17)$ & $1537(13)$ & $8934(10)$ & $58(5)$ \\
$\mathrm{C}(4)$ & $5877(13)$ & $1379(11)$ & $8758(9)$ & $57(3)$ \\
$\mathrm{C}(5)$ & $6745(15)$ & $2061(13)$ & $8682(10)$ & $82(4)$ \\
$\mathrm{O}(6)$ & $6580(12)$ & $3195(10)$ & $8844(7)$ & $50(3)$ \\
$\mathrm{O}(7)$ & $5176(10)$ & $3481(6)$ & $8744(5)$ & $49(2)$ \\
$\mathrm{C}(8)$ & $3145(11)$ & $3032(9)$ & $8715(6)$ & $70(3)$ \\
$\mathrm{C}(9)$ & $2910(14)$ & $4166(11)$ & $8575(8)$ & $73(4)$ \\
$\mathrm{C}(10)$ & $1464(18)$ & $4294(14)$ & $8532(10)$ & $87(5)$ \\
$\mathrm{C}(11)$ & $831(19)$ & $4001(16)$ & $9111(10)$ & $79(5)$ \\
$\mathrm{C}(12)$ & $1045(19)$ & $5441(14)$ & $8198(10)$ & $82(5)$ \\
$\mathrm{C}(13)$ & $1332(18)$ & $6365(14)$ & $8748(10)$ & $61(5)$ \\
$\mathrm{O}(14)$ & $980(30)$ & $7450(20)$ & $8394(15)$ & $64(8)$ \\
$\mathrm{O}(15)$ & $726(19)$ & $7763(16)$ & $7908(12)$ & $107(7)$ \\
$\mathrm{C}(16)$ & $601(14)$ & $8290(10)$ & $8993(7)$ & $77(4)$ \\
$\mathrm{C}(17)$ & $183(17)$ & $9350(13)$ & $8726(10)$ & $79(4)$ \\
$\mathrm{C}(18)$ & $1328(15)$ & $10065(13)$ & $8901(9)$ & $71(4)$ \\
$\mathrm{C}(1 \mathrm{~B})$ & $8185(18)$ & $1751(16)$ & $8995(10)$ & $94(5)$ \\
$\mathrm{C}(2 \mathrm{~B})$ & $4037(12)$ & $2629(8)$ & $8638(6)$ & $53(3)$ \\
$\mathrm{C}(3 \mathrm{~B})$ & $4431(12)$ & $1572(10)$ & $8810(7)$ & $62(3)$ \\
$\mathrm{C}(4 \mathrm{~B})$ & $5983(13)$ & $1392(11)$ & $9028(8)$ & $88(4)$ \\
$\mathrm{C}(5 \mathrm{~B})$ & $6726(10)$ & $2301(8)$ & $9146(7)$ & $73(3)$ \\
$\mathrm{O}(6 \mathrm{~B})$ & $6150(11)$ & $3408(8)$ & $8667(6)$ & $66(3)$ \\
& $4697(7)$ & $3458(5)$ & $8586(4)$ & $55(2)$ \\
& & & &
\end{tabular}




$\begin{array}{lcccr}\mathrm{O}(7 \mathrm{~B}) & 2805(7) & 2756(6) & 8471(4) & 70(2) \\ \mathrm{C}(8 \mathrm{~B}) & 2284(10) & 3788(8) & 8186(5) & 72(3) \\ \mathrm{C}(9 \mathrm{~B}) & 1996(10) & 4569(7) & 8786(5) & 61(2) \\ \mathrm{C}(10 B) & 1120(13) & 4135(11) & 9322(7) & 81(4) \\ \mathrm{C}(11 \mathrm{~B}) & 1463(10) & 5585(8) & 8385(5) & 56(2) \\ \mathrm{C}(12 B) & 1274(13) & 6544(10) & 8892(7) & 63(3) \\ \mathrm{C}(13 \mathrm{~B}) & 864(16) & 7546(13) & 8468(9) & 55(5) \\ \mathrm{O}(14 \mathrm{~B}) & 662(8) & 7574(7) & 7761(5) & 61(2) \\ \mathrm{O}(15 B) & 934(9) & 8300(7) & 8868(5) & 73(2) \\ \mathrm{C}(16 \mathrm{~B}) & 631(13) & 9359(9) & 8532(7) & 81(3) \\ \mathrm{C}(17 \mathrm{~B}) & 943(12) & 10156(10) & 9111(7) & 83(3) \\ \mathrm{C}(18 B) & 8155(16) & 2272(13) & 9131(9) & 110(5)\end{array}$


Table 3. Bond lengths $[\AA]$ and angles $\left[{ }^{\circ}\right]$ for kaw64.

\begin{tabular}{ll}
\hline $\mathrm{Sb}(1)-\mathrm{Cl}(2)$ & $2.3436(13)$ \\
$\mathrm{Sb}(1)-\mathrm{Cl}(1)$ & $2.3497(13)$ \\
$\mathrm{Sb}(1)-\mathrm{Cl}(3)$ & $2.3528(14)$ \\
$\mathrm{Sb}(1)-\mathrm{Cl}(5)$ & $2.3609(11)$ \\
$\mathrm{Sb}(1)-\mathrm{Cl}(4)$ & $2.3691(13)$ \\
$\mathrm{Sb}(1)-\mathrm{Cl}(6)$ & $2.3753(11)$ \\
$\mathrm{C}(1)-\mathrm{O}(6)$ & $1.319(18)$ \\
$\mathrm{C}(1)-\mathrm{O}(7)$ & $1.33(2)$ \\
$\mathrm{C}(1)-\mathrm{C}(2)$ & $1.46(2)$ \\
$\mathrm{C}(2)-\mathrm{C}(3)$ & $1.30(2)$ \\
$\mathrm{C}(3)-\mathrm{C}(4)$ & $1.25(2)$ \\
$\mathrm{C}(4)-\mathrm{C}(5)$ & $1.44(2)$ \\
$\mathrm{C}(4)-\mathrm{C}(18)$ & $1.58(2)$ \\
$\mathrm{C}(5)-\mathrm{O}(6)$ & $1.493(15)$ \\
$\mathrm{O}(7)-\mathrm{C}(8)$ & $1.434(16)$ \\
$\mathrm{C}(8)-\mathrm{C}(9)$ & $1.51(2)$ \\
$\mathrm{C}(9)-\mathrm{C}(10)$ & $1.36(2)$ \\
$\mathrm{C}(9)-\mathrm{C}(11)$ & $1.58(2)$ \\
$\mathrm{C}(11)-\mathrm{C}(12)$ & $1.52(2)$ \\
$\mathrm{C}(12)-\mathrm{C}(13)$ & $1.50(3)$ \\
$\mathrm{C}(13)-\mathrm{O}(14)$ & $0.98(3)$ \\
$\mathrm{C}(13)-\mathrm{O}(15)$ & $1.59(3)$ \\
$\mathrm{O}(15)-\mathrm{C}(16)$ & $1.44(2)$ \\
$\mathrm{C}(16)-\mathrm{C}(17)$ & $1.48(2)$ \\
$\mathrm{C}(1 \mathrm{~B})-\mathrm{O}(6 \mathrm{~B})$ & $1.240(12)$ \\
$\mathrm{C}(1 \mathrm{~B})-\mathrm{O}(7 \mathrm{~B})$ & $1.290(14)$ \\
$\mathrm{C}(1 \mathrm{~B})-\mathrm{C}(2 \mathrm{~B})$ & $1.388(15)$ \\
$\mathrm{C}(2 \mathrm{~B})-\mathrm{C}(3 \mathrm{~B})$ & $1.631(18)$ \\
$\mathrm{C}(3 \mathrm{~B})-\mathrm{C}(4 \mathrm{~B})$ & $1.363(16)$ \\
$\mathrm{C}(4 \mathrm{~B})-\mathrm{C}(18 \mathrm{~B})$ & $1.492(18)$ \\
$\mathrm{C}(4 \mathrm{~B})-\mathrm{C}(5 \mathrm{~B})$ & $1.689(15)$ \\
$\mathrm{C}(5 \mathrm{~B})-\mathrm{O}(6 \mathrm{~B})$ & $1.503(12)$ \\
$\mathrm{O}(7 \mathrm{~B})-\mathrm{C}(8 \mathrm{~B})$ & $1.453(11)$ \\
$\mathrm{C}(8 \mathrm{~B})-\mathrm{C}(9 \mathrm{~B})$ & \\
& \\
&
\end{tabular}




\begin{tabular}{|c|c|}
\hline C(9B)-C(10B) & $1.515(16)$ \\
\hline $\mathrm{C}(9 \mathrm{~B})-\mathrm{C}(11 \mathrm{~B})$ & $1.520(12)$ \\
\hline $\mathrm{C}(11 \mathrm{~B})-\mathrm{C}(12 \mathrm{~B})$ & $1.527(15)$ \\
\hline $\mathrm{C}(12 \mathrm{~B})-\mathrm{C}(13 \mathrm{~B})$ & $1.49(2)$ \\
\hline$C(13 B)-O(15 B)$ & $1.179(17)$ \\
\hline $\mathrm{C}(13 \mathrm{~B})-\mathrm{O}(14 \mathrm{~B})$ & $1.285(19)$ \\
\hline $\mathrm{O}(15 \mathrm{~B})-\mathrm{C}(16 \mathrm{~B})$ & $1.458(14)$ \\
\hline $\mathrm{C}(16 \mathrm{~B})-\mathrm{C}(17 \mathrm{~B})$ & $1.450(16)$ \\
\hline $\mathrm{Cl}(2)-\mathrm{Sb}(1)-\mathrm{Cl}(1)$ & $179.75(6)$ \\
\hline $\mathrm{Cl}(2)-\mathrm{Sb}(1)-\mathrm{Cl}(3)$ & $90.90(7)$ \\
\hline $\mathrm{Cl}(1)-\mathrm{Sb}(1)-\mathrm{Cl}(3)$ & $89.30(7)$ \\
\hline $\mathrm{Cl}(2)-\mathrm{Sb}(1)-\mathrm{Cl}(5)$ & $89.12(5)$ \\
\hline $\mathrm{Cl}(1)-\mathrm{Sb}(1)-\mathrm{Cl}(5)$ & $90.73(5)$ \\
\hline $\mathrm{Cl}(3)-\mathrm{Sb}(1)-\mathrm{Cl}(5)$ & $91.01(5)$ \\
\hline $\mathrm{Cl}(2)-\mathrm{Sb}(1)-\mathrm{Cl}(4)$ & $90.23(6)$ \\
\hline $\mathrm{Cl}(1)-\mathrm{Sb}(1)-\mathrm{Cl}(4)$ & $89.57(6)$ \\
\hline $\mathrm{Cl}(3)-\mathrm{Sb}(1)-\mathrm{Cl}(4)$ & $178.82(6)$ \\
\hline $\mathrm{Cl}(5)-\mathrm{Sb}(1)-\mathrm{Cl}(4)$ & $89.36(5)$ \\
\hline $\mathrm{Cl}(2)-\mathrm{Sb}(1)-\mathrm{Cl}(6)$ & $90.17(5)$ \\
\hline $\mathrm{Cl}(1)-\mathrm{Sb}(1)-\mathrm{Cl}(6)$ & $89.98(5)$ \\
\hline $\mathrm{Cl}(3)-\mathrm{Sb}(1)-\mathrm{Cl}(6)$ & $90.33(5)$ \\
\hline $\mathrm{Cl}(5)-\mathrm{Sb}(1)-\mathrm{Cl}(6)$ & $178.49(4)$ \\
\hline $\mathrm{Cl}(4)-\mathrm{Sb}(1)-\mathrm{Cl}(6)$ & $89.32(5)$ \\
\hline $\mathrm{O}(6)-\mathrm{C}(1)-\mathrm{O}(7)$ & $110.6(13)$ \\
\hline $\mathrm{O}(6)-\mathrm{C}(1)-\mathrm{C}(2)$ & $125.4(15)$ \\
\hline $\mathrm{O}(7)-\mathrm{C}(1)-\mathrm{C}(2)$ & $123.8(14)$ \\
\hline $\mathrm{C}(3)-\mathrm{C}(2)-\mathrm{C}(1)$ & $108.5(15)$ \\
\hline $\mathrm{C}(4)-\mathrm{C}(3)-\mathrm{C}(2)$ & $129.1(15)$ \\
\hline $\mathrm{C}(3)-\mathrm{C}(4)-\mathrm{C}(5)$ & $121.3(14)$ \\
\hline $\mathrm{C}(3)-\mathrm{C}(4)-\mathrm{C}(18)$ & $117.5(15)$ \\
\hline$C(5)-C(4)-C(18)$ & $106.9(14)$ \\
\hline $\mathrm{C}(4)-\mathrm{C}(5)-\mathrm{O}(6)$ & $110.0(10)$ \\
\hline $\mathrm{C}(1)-\mathrm{O}(6)-\mathrm{C}(5)$ & $116.3(10)$ \\
\hline $\mathrm{C}(1)-\mathrm{O}(7)-\mathrm{C}(8)$ & $118.8(11)$ \\
\hline $\mathrm{O}(7)-\mathrm{C}(8)-\mathrm{C}(9)$ & $105.0(12)$ \\
\hline
\end{tabular}




$\begin{array}{ll}\mathrm{C}(10)-\mathrm{C}(9)-\mathrm{C}(8) & 120.4(16) \\ \mathrm{C}(10)-\mathrm{C}(9)-\mathrm{C}(11) & 113.6(16) \\ \mathrm{C}(8)-\mathrm{C}(9)-\mathrm{C}(11) & 110.0(14) \\ \mathrm{C}(12)-\mathrm{C}(11)-\mathrm{C}(9) & 112.9(15) \\ \mathrm{C}(13)-\mathrm{C}(12)-\mathrm{C}(11) & 111.0(17) \\ \mathrm{O}(14)-\mathrm{C}(13)-\mathrm{C}(12) & 140(3) \\ \mathrm{O}(14)-\mathrm{C}(13)-\mathrm{O}(15) & 108(2) \\ \mathrm{C}(12)-\mathrm{C}(13)-\mathrm{O}(15) & 110.4(19) \\ \mathrm{C}(16)-\mathrm{O}(15)-\mathrm{C}(13) & 116.6(15) \\ \mathrm{O}(15)-\mathrm{C}(16)-\mathrm{C}(17) & 105.2(13) \\ \mathrm{O}(6 \mathrm{~B})-\mathrm{C}(1 \mathrm{~B})-\mathrm{O}(7 \mathrm{~B}) & 115.2(9) \\ \mathrm{O}(6 \mathrm{~B})-\mathrm{C}(1 \mathrm{~B})-\mathrm{C}(2 \mathrm{~B}) & 129.6(11) \\ \mathrm{O}(7 \mathrm{~B})-\mathrm{C}(1 \mathrm{~B})-\mathrm{C}(2 \mathrm{~B}) & 115.2(10) \\ \mathrm{C}(1 \mathrm{~B})-\mathrm{C}(2 \mathrm{~B})-\mathrm{C}(3 \mathrm{~B}) & 116.1(10) \\ \mathrm{C}(4 \mathrm{~B})-\mathrm{C}(3 \mathrm{~B})-\mathrm{C}(2 \mathrm{~B}) & 116.9(10) \\ \mathrm{C}(3 \mathrm{~B})-\mathrm{C}(4 \mathrm{~B})-\mathrm{C}(18 \mathrm{~B}) & 121.7(11) \\ \mathrm{C}(3 \mathrm{~B})-\mathrm{C}(4 \mathrm{~B})-\mathrm{C}(5 \mathrm{~B}) & 114.9(10) \\ \mathrm{C}(18 \mathrm{~B})-\mathrm{C}(4 \mathrm{~B})-\mathrm{C}(5 \mathrm{~B}) & 107.6(9) \\ \mathrm{O}(6 \mathrm{~B})-\mathrm{C}(5 \mathrm{~B})-\mathrm{C}(4 \mathrm{~B}) & 112.0(7) \\ \mathrm{C}(1 \mathrm{~B})-\mathrm{O}(6 \mathrm{~B})-\mathrm{C}(5 \mathrm{~B}) & 121.2(8) \\ \mathrm{C}(1 \mathrm{~B})-\mathrm{O}(7 \mathrm{~B})-\mathrm{C}(8 \mathrm{~B}) & 120.5(8) \\ \mathrm{O}(7 \mathrm{~B})-\mathrm{C}(8 \mathrm{~B})-\mathrm{C}(9 \mathrm{~B}) & 113.0(8) \\ \mathrm{C}(10 \mathrm{~B})-\mathrm{C}(9 \mathrm{~B})-\mathrm{C}(8 \mathrm{~B}) & 115.7(9) \\ \mathrm{C}(10 \mathrm{~B})-\mathrm{C}(9 \mathrm{~B})-\mathrm{C}(11 \mathrm{~B}) & 112.9(9) \\ \mathrm{C}(8 \mathrm{~B})-\mathrm{C}(9 \mathrm{~B})-\mathrm{C}(11 \mathrm{~B}) & 105.3(7) \\ \mathrm{C}(9 \mathrm{~B})-\mathrm{C}(11 \mathrm{~B})-\mathrm{C}(12 \mathrm{~B}) & 114.2(8) \\ \mathrm{C}(13 \mathrm{~B})-\mathrm{C}(12 \mathrm{~B})-\mathrm{C}(11 \mathrm{~B}) & 111.9(11) \\ \mathrm{O}(15 \mathrm{~B})-\mathrm{C}(13 \mathrm{~B})-\mathrm{O}(14 \mathrm{~B}) & 126.4(15) \\ \mathrm{O}(15 \mathrm{~B})-\mathrm{C}(13 \mathrm{~B})-\mathrm{C}(12 \mathrm{~B}) & 109.6(14) \\ \mathrm{O}(14 \mathrm{~B})-\mathrm{C}(13 \mathrm{~B})-\mathrm{C}(12 \mathrm{~B}) & 123.2(13) \\ \mathrm{C}(13 \mathrm{~B})-\mathrm{O}(15 \mathrm{~B})-\mathrm{C}(16 \mathrm{~B}) & 116.6(11) \\ \mathrm{C}(17 \mathrm{~B})-\mathrm{C}(16 \mathrm{~B})-\mathrm{O}(15 \mathrm{~B}) & 106.2(9) \\ & \end{array}$


Table 4. Anisotropic displacement parameters $\left(\AA^{2} \times 10^{3}\right)$ for kaw64. The anisotropic displacement factor exponent takes the form: $-2 \pi^{2}\left[h^{2} a^{* 2} U^{11}+\ldots+2 h k a^{*} b^{*} U^{12}\right]$

\begin{tabular}{lcccccc}
\hline & $\mathrm{U}^{11}$ & $\mathrm{U}^{22}$ & $\mathrm{U}^{33}$ & $\mathrm{U}^{23}$ & $\mathrm{U}^{13}$ & $\mathrm{U}^{12}$ \\
\hline $\mathrm{Sb}(1)$ & $49(1)$ & $42(1)$ & $41(1)$ & $-1(1)$ & $12(1)$ & $-5(1)$ \\
$\mathrm{Cl}(1)$ & $97(1)$ & $87(1)$ & $66(1)$ & $-8(1)$ & $-7(1)$ & $-34(1)$ \\
$\mathrm{Cl}(2)$ & $105(1)$ & $83(1)$ & $62(1)$ & $2(1)$ & $-13(1)$ & $-36(1)$ \\
$\mathrm{Cl}(3)$ & $80(1)$ & $74(1)$ & $122(1)$ & $9(1)$ & $50(1)$ & $19(1)$ \\
$\mathrm{Cl}(4)$ & $84(1)$ & $63(1)$ & $106(1)$ & $8(1)$ & $47(1)$ & $17(1)$ \\
$\mathrm{Cl}(5)$ & $83(1)$ & $64(1)$ & $54(1)$ & $11(1)$ & $22(1)$ & $-12(1)$ \\
$\mathrm{Cl}(6)$ & $87(1)$ & $54(1)$ & $57(1)$ & $10(1)$ & $23(1)$ & $-10(1)$ \\
\hline
\end{tabular}


Table 5. Hydrogen coordinates ( $\left.\times 10^{4}\right)$ and isotropic displacement parameters $\left(\AA^{2} \times 10^{3}\right)$ for kaw64.

\begin{tabular}{|c|c|c|c|c|}
\hline & $\mathrm{x}$ & $\mathrm{y}$ & $\mathrm{z}$ & $\mathrm{U}(\mathrm{eq})$ \\
\hline $\mathrm{H}(2 \mathrm{~A})$ & 4702 & 1383 & 9458 & 69 \\
\hline $\mathrm{H}(2 \mathrm{~B})$ & 4104 & 1050 & 8649 & 69 \\
\hline $\mathrm{H}(3 \mathrm{~A})$ & 6278 & 861 & 9124 & 69 \\
\hline $\mathrm{H}(3 \mathrm{~B})$ & 5748 & 983 & 8290 & 69 \\
\hline $\mathrm{H}(4 \mathrm{~A})$ & 6772 & 2063 & 8143 & 99 \\
\hline $\mathrm{H}(5 \mathrm{~A})$ & 7040 & 3640 & 8515 & 61 \\
\hline $\mathrm{H}(5 \mathrm{~B})$ & 6950 & 3346 & 9352 & 61 \\
\hline $\mathrm{H}(8 \mathrm{~A})$ & 3216 & 4384 & 8111 & 87 \\
\hline $\mathrm{H}(8 \mathrm{~B})$ & 3350 & 4609 & 8975 & 87 \\
\hline $\mathrm{H}(9 \mathrm{~A})$ & 1127 & 3779 & 8138 & 104 \\
\hline $\mathrm{H}(10 \mathrm{~A})$ & 1171 & 3314 & 9309 & 118 \\
\hline $\mathrm{H}(10 \mathrm{~B})$ & -86 & 3926 & 8945 & 118 \\
\hline $\mathrm{H}(10 \mathrm{C})$ & 958 & 4556 & 9489 & 118 \\
\hline $\mathrm{H}(11 \mathrm{~A})$ & 1501 & 5578 & 7769 & 99 \\
\hline $\mathrm{H}(11 \mathrm{~B})$ & 115 & 5430 & 8029 & 99 \\
\hline $\mathrm{H}(12 \mathrm{~A})$ & 840 & 6255 & 9165 & 73 \\
\hline $\mathrm{H}(12 \mathrm{~B})$ & 2255 & 6361 & 8936 & 73 \\
\hline $\mathrm{H}(16 \mathrm{~A})$ & -543 & 9607 & 8974 & 95 \\
\hline $\mathrm{H}(16 \mathrm{~B})$ & -88 & 9331 & 8194 & 95 \\
\hline $\mathrm{H}(17 \mathrm{~A})$ & 1107 & 10800 & 8741 & 107 \\
\hline $\mathrm{H}(17 \mathrm{~B})$ & 2033 & 9802 & 8647 & 107 \\
\hline $\mathrm{H}(17 \mathrm{C})$ & 1593 & 10059 & 9428 & 107 \\
\hline $\mathrm{H}(18 \mathrm{~A})$ & 8339 & 992 & 8892 & 141 \\
\hline $\mathrm{H}(18 \mathrm{~B})$ & 8315 & 1871 & 9523 & 141 \\
\hline $\mathrm{H}(18 \mathrm{C})$ & 8784 & 2200 & 8761 & 141 \\
\hline $\mathrm{H}(2 \mathrm{BA})$ & 4142 & 1108 & 8387 & 74 \\
\hline $\mathrm{H}(2 \mathrm{BB})$ & 3989 & 1324 & 9223 & 74 \\
\hline $\mathrm{H}(3 \mathrm{BA})$ & 6110 & 947 & 9476 & 105 \\
\hline $\mathrm{H}(3 \mathrm{BB})$ & 6306 & 972 & 8634 & 105 \\
\hline $\mathrm{H}(4 \mathrm{BA})$ & 6638 & 2496 & 9663 & 87 \\
\hline $\mathrm{H}(5 \mathrm{BA})$ & 6504 & 4064 & 8921 & 79 \\
\hline
\end{tabular}




$\begin{array}{lrrrr}\mathrm{H}(5 \mathrm{BB}) & 6440 & 3393 & 8178 & 79 \\ \mathrm{H}(8 \mathrm{BA}) & 1485 & 3654 & 7856 & 86 \\ \mathrm{H}(8 \mathrm{BB}) & 2906 & 4128 & 7895 & 86 \\ \mathrm{H}(9 \mathrm{BA}) & 2832 & 4765 & 9074 & 73 \\ \mathrm{H}(10 \mathrm{D}) & 1514 & 3500 & 9571 & 121 \\ \mathrm{H}(10 \mathrm{E}) & 289 & 3937 & 9055 & 121 \\ \mathrm{H}(10 \mathrm{~F}) & 994 & 4691 & 9682 & 121 \\ \mathrm{H}(11 \mathrm{C}) & 629 & 5409 & 8102 & 67 \\ \mathrm{H}(11 \mathrm{D}) & 2056 & 5805 & 8035 & 67 \\ \mathrm{H}(12 \mathrm{C}) & 617 & 6355 & 9211 & 76 \\ \mathrm{H}(12 \mathrm{D}) & 2087 & 6687 & 9207 & 76 \\ \mathrm{H}(16 \mathrm{C}) & 1148 & 9486 & 8127 & 97 \\ \mathrm{H}(16 \mathrm{D}) & -288 & 9400 & 8339 & 97 \\ \mathrm{H}(17 \mathrm{D}) & 743 & 10878 & 8917 & 124 \\ \mathrm{H}(17 \mathrm{E}) & 1857 & 10112 & 9291 & 124 \\ \mathrm{H}(17 \mathrm{~F}) & 437 & 10010 & 9511 & 124 \\ \mathrm{H}(18 \mathrm{D}) & 8516 & 1646 & 9406 & 165 \\ \mathrm{H}(18 \mathrm{E}) & 8539 & 2931 & 9352 & 165 \\ \mathrm{H}(18 \mathrm{~F}) & 8340 & 2220 & 8625 & 165\end{array}$


IVD. Relevant ${ }^{1} \mathrm{H}$ NMR Coupling Constant Data for 5, 6, S7, and 9

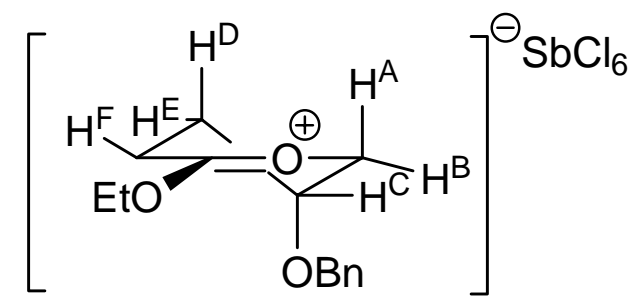

1-Ethoxy-4-(benzyloxy)-2,3,4,5-tetrahydropyrylium hexachloroantimonate (5)

$\mathbf{H}^{\mathrm{A}}: \mathrm{dd}, J=12.3(\mathrm{gem}), 2.0\left(\mathrm{ax}^{\mathrm{A}}-\mathrm{eq}^{\mathrm{C}}\right)$

$\mathbf{H}^{\mathrm{B}}:$ ddd, $J=12.3(\mathrm{gem}), 2.6\left(\right.$ remote coupling to $\left.\mathrm{H}^{\mathrm{F}}\right), 2.0\left(\mathrm{eq}^{\mathrm{B}}-\mathrm{eq}^{\mathrm{C}}\right)$

$\mathbf{H}^{\mathrm{C}}$ : sextet, $J=2.0$ (vicinal coupling to $\mathrm{H}^{\mathrm{A}}, \mathrm{H}^{\mathrm{B}}, \mathrm{H}^{\mathrm{D}}$, and $\mathrm{H}^{\mathrm{E}}$, and remote coupling to $\mathrm{H}^{\mathrm{F}}$ )

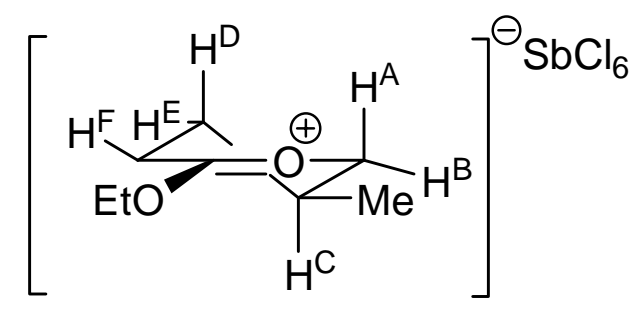

\section{1-Ethoxy-4-methyl-2,3,4,5-tetrahydropyrylium hexachloroantimonate (6)}

$\mathbf{H}^{\mathrm{A}}: \mathrm{t}, J=11.2(\mathrm{gem}), 11.2\left(\mathrm{ax}^{\mathrm{A}}-\mathrm{ax}^{\mathrm{C}}\right)$

$\mathbf{H}^{\mathrm{B}}: \operatorname{ddd}, J=11.2(\mathrm{gem}), 5.0\left(\mathrm{eq}^{\mathrm{B}}-\mathrm{eq}^{\mathrm{C}}\right), 2.0\left(\right.$ remote coupling to $\left.\mathrm{H}^{\mathrm{F}}\right)$

$\mathbf{H}^{\mathrm{C}}:$ ddqdd, $J=14.1\left(\mathrm{ax}^{\mathrm{C}}-\mathrm{ax}^{\mathrm{D}}\right), 11.2\left(\mathrm{ax}^{\mathrm{C}}-\mathrm{ax}^{\mathrm{A}}\right), 6.7\left(\mathrm{ax}^{\mathrm{C}}-\mathrm{Me}\right), 6.2\left(\mathrm{ax}^{\mathrm{C}}-\mathrm{eq}^{\mathrm{E}}\right), 5.0\left(\mathrm{ax}^{\mathrm{C}}-\right.$ $\left.e q^{B}\right)$

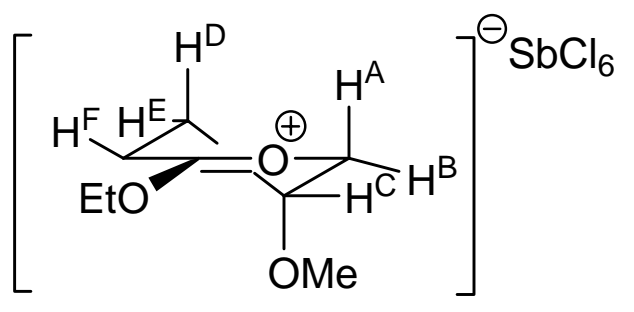

1-Ethoxy-4-methoxy-2,3,4,5-tetrahydropyrylium hexachloroantimonate (S7) 
$\mathbf{H}^{\mathrm{A}}: \mathrm{dd}, J=12.3(\mathrm{gem}), 2.0\left(\mathrm{ax}^{\mathrm{A}}-\mathrm{eq}^{\mathrm{C}}\right)$

$\mathbf{H}^{\mathrm{B}}:$ ddd, $J=12.3(\mathrm{gem}), 2.0\left(\mathrm{eq}^{\mathrm{B}}-\mathrm{eq}^{\mathrm{C}}\right), 1.4\left(\right.$ remote coupling to $\left.\mathrm{H}^{\mathrm{F}}\right)$

$\mathbf{H}^{\mathrm{C}}$ : sextet, $J=2.0$ (vicinal coupling to $\mathrm{H}^{\mathrm{A}}, \mathrm{H}^{\mathrm{B}}, \mathrm{H}^{\mathrm{D}}$, and $\mathrm{H}^{\mathrm{E}}$, and remote coupling to $\mathrm{H}^{\mathrm{F}}$ )

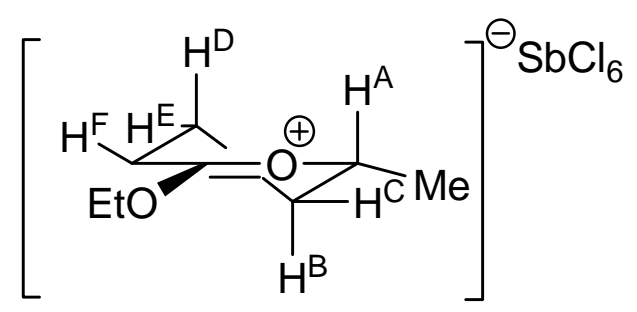

\section{1-Ethoxy-5-methyl-2,3,4,5-tetrahydropyrylium hexachloroantimonate $(9)^{10}$}

$\mathbf{H}^{\mathrm{A}}: \mathrm{dqd}, J=10.6\left(\mathrm{ax}^{\mathrm{A}}-\mathrm{ax}^{\mathrm{C}}\right), 6.5\left(\mathrm{ax}^{\mathrm{A}}-\mathrm{Me}\right), 3.1\left(\mathrm{ax}^{\mathrm{A}}-\mathrm{eq}^{\mathrm{C}}\right)$

$\mathbf{H}^{\mathrm{B}}: \operatorname{dddd}, J=15.1(\mathrm{gem}), 11.4\left(\mathrm{ax}^{\mathrm{B}}-\mathrm{ax}^{\mathrm{D}}\right), 10.6\left(\mathrm{ax}^{\mathrm{B}}-\mathrm{ax}^{\mathrm{A}}\right), 4.6\left(\mathrm{ax}^{\mathrm{B}}-\mathrm{eq}^{\mathrm{E}}\right)$

$\mathbf{H}^{\mathrm{C}}$ : dtdd, $J=15.1(\mathrm{gem}), 4.0\left(\mathrm{eq}^{\mathrm{C}}-\mathrm{ax}^{\mathrm{D}}\right.$ and $\left.\mathrm{eq}^{\mathrm{C}}-\mathrm{eq}^{\mathrm{E}}\right), 3.1\left(\mathrm{eq}^{\mathrm{C}}-\mathrm{ax}^{\mathrm{A}}\right), 1.2\left(\mathrm{eq}^{\mathrm{C}}-\mathrm{eq}^{\mathrm{F}}\right)$

*Because most $J$ values for this cation have not been determined, complete ${ }^{1} \mathrm{H}$ NMR data appears below:

${ }^{1} \mathrm{H}$ NMR (500 MHz, $\mathrm{CD}_{2} \mathrm{Cl}_{2}$, referenced to $\left.\delta 5.32\right) \delta 5.52(\mathrm{dqd}, J=10.6,6.5,3.1,1 \mathrm{H})$, $4.90(\mathrm{dq}, J=10.6,7.1,1 \mathrm{H}), 4.84(\mathrm{dq}, J=10.6,7.1,1 \mathrm{H}), 3.23(\mathrm{dddd}, J=21.2,6.8,3.6$, $1.2,1 \mathrm{H}), 3.08$ (ddd, $J=21.2,9.3,7.5,1 \mathrm{H}), 2.37(\mathrm{dtdd}, J=15.1,4.0,3.1,1.2,1 \mathrm{H}), 2.24-$ 2.11 (ddddd, $J=14.2,7.5,4.6,4.0,3.6,1 \mathrm{H}$, and ddddd, $J=14.2,11.4,9.3,6.8,4.0,1 \mathrm{H}$ ), 1.95 (dddd, $J=15.1,11.4,10.6,4.6,1 \mathrm{H}), 1.75(\mathrm{t}, J=6.5,3 \mathrm{H}), 1.58(\mathrm{~d}, J=7.1,3 \mathrm{H})$.

*This high-field ${ }^{1} \mathrm{H}$ NMR data is most consistent with a pseudoequatorial C-5 methyl group. Childs has proven the pseudoequatorial orientation of the C-5 methyl group using X-ray crystallography. ${ }^{10}$ 


\section{Computational Studies on 4-Alkoxy- and 4-Methyl-2,3,4,5- tetrahydropyrylium Cations}

Method: Cations were initially drawn in half-chair conformations with the lowest energy (Z)-relationship between the ethyl group and $\mathrm{O} 1$ about the $\mathrm{C} 1-\mathrm{O} 2$ partial double bond. Substituents at C-4 were placed pseudoaxially or pseudoequatorially, then equilibrium geometries were determined at the MP2/6-31G* level of theory using Spartan '02. ${ }^{15}$

Note: In our discussion of anchimeric assistance in the text of the Communication, we discounted the involvement of bridged bicyclic oxonium ion 11. We carried out several theoretical analyses of $\mathbf{1 1}$, and it was only found to be a minimum energy structure using molecular mechanics. Geometry optimizations performed at higher (and more relevant) levels of theory (HF, DFT, and MP2 correlation) revealed that only structures similar to $\mathbf{4}$ were low in energy. To our knowledge, our study is the first to employ theoretical methods to study the equilibrium between monocyclic dioxocarbenium ion $\mathbf{4}$ and the charged, bridged bicyclic ortho ester 11.

Similar bridged bicyclic structures have been proposed in the literature for both charged and neutral species. In his total synthesis of (+)-lepicidin A, Evans prepared a 1deoxy-aza-analogue of $\mathbf{1 1}$ by trapping an oxocarbenium ion intermediate with a remote nitrogen substituent. ${ }^{16}$ In dioxocarbenium ion $\mathbf{5}$, however, a bonding interaction between C-1 and the remote alkoxy substituent should be disfavored because the oxonium ion is higher in energy. Our computational results suggest that this assumption is valid.

Acid-catalyzed ring opening of bridged bicyclic ortho esters is considerably slower than ring opening of the analogous 2,7-dioxabicyclo[2.2.1]heptane because the former do not experience as much ring strain. ${ }^{17}$ The ring strain present in 2,7- 
dioxabicyclo[2.2.1] heptanes is sizable, and hydrolyses of these systems experience a rate acceleration attributable to relief of this ring strain. ${ }^{17}$ Furthermore, because we do not observe a bridged bicyclic structure in an ortho ester derivative (where it should be more stable), it should not occur in oxocarbenium ion intermediates like 2.

\section{- 4-Methoxytetrahydropyrylium ion}

Pseudoaxial 4-Methoxytetrahydropyrylium ion, S8ax, MP2/6-31G* Equilibrium Geometry

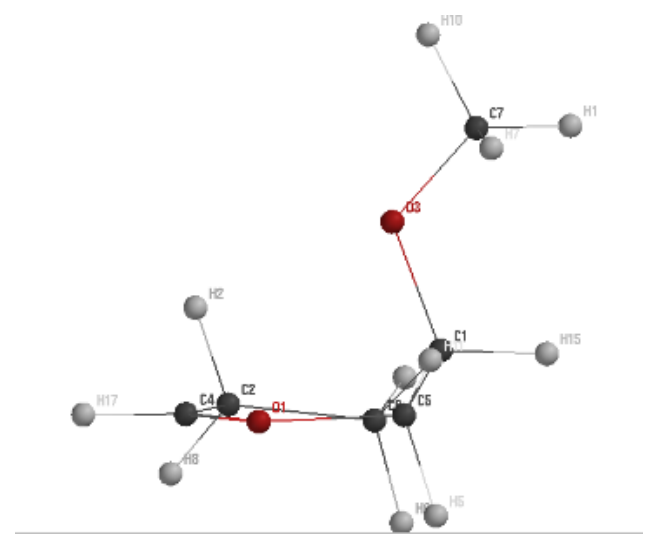

SPARTAN 'O2 Quantum Mechanics Program: (SGI/R10K)

Job type: Geometry optimization.

Method: RMP2(FC)

Basis set: 6-31G(D)

Number of shells: 54

Number of basis functions: 142

SCF model:

A restricted Hartree-Fock SCF calculation will be performed using Pulay DIIS extrapolation

Correlation model:

A MP2 calculation will be performed

Optimization:

\begin{tabular}{ccrr} 
Step & Energy & Max Grad. & \multicolumn{1}{c}{ Max Dist. } \\
1 & -384.1773771 & 0.017825 & 0.068006 \\
2 & -384.1800654 & 0.004561 & 0.048706 \\
3 & -384.1801972 & 0.000473 & 0.014964 \\
4 & -384.1802099 & 0.000301 & 0.025708 \\
5 & -384.1802142 & 0.000110 & 0.010969
\end{tabular}




$$
\begin{array}{llll}
6 & -384.1802154 & 0.000096 & 0.006471 \\
7 & -384.1802158 & 0.000030 & 0.002129
\end{array}
$$

\begin{tabular}{|c|c|c|c|}
\hline \multicolumn{4}{|c|}{ Coordinates (Angstroms) } \\
\hline & M $\quad$ X & $\mathrm{Y}$ & $\mathrm{Z}$ \\
\hline $1 \mathrm{H}$ & -1.072179 & 0.152781 & 1.563886 \\
\hline $2 \mathrm{C}$ & -0.401193 & & 0.697921 \\
\hline $3 \mathrm{C}$ & 730526 & 0.693326 & -0.357364 \\
\hline $4 \mathrm{H}$ & 18000 & 1.8 & \\
\hline $5 \mathrm{C}$ & 561024 & -0.6 & -0.9 \\
\hline $6 \mathrm{C}$ & -0.094598 & -1.418003 & 0.50 \\
\hline $7 \mathrm{C}$ & & & \\
\hline $8 \mathrm{H}$ & & & \\
\hline $9 \mathrm{H}$ & & & \\
\hline $0 \mathrm{C}$ & & & -0.5 \\
\hline $1 \mathrm{H}$ & & & \\
\hline $12 \mathrm{I}$ & & & 1.7 \\
\hline 13( & & & \\
\hline $14 \mathrm{H}$ & & & -0.2 \\
\hline 150 & 729 & -1.5 & -0.6 \\
\hline $16 \mathrm{H}$ & -2.895983 & 0.907984 & \\
\hline $17 \mathrm{H}$ & -2.679741 & -0.647435 & -0.605042 \\
\hline $18 \mathrm{H}$ & & & \\
\hline & 2.286117 & -0.904502 & -1.826028 \\
\hline
\end{tabular}

Dipole Moment (Debye) Tot $\quad 3.5662$

Point Group: c1 Number of degrees of freedom: 51

\section{Energy is $\mathbf{- 3 8 4 . 1 8 0 2 1 5 7 7 8}$ hartrees}

Pseudoequatorial 4-Methoxytetrahydropyrylium ion, S8eq, MP2/6-31G* Equilibrium Geometry

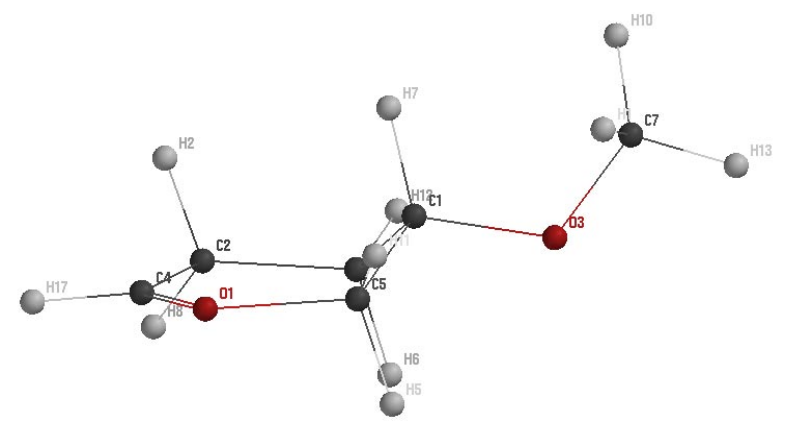

SPARTAN 'O2 Quantum Mechanics Program: (SGI/R10K)

Job type: Geometry optimization.

Method: RMP2(FC)

Basis set: 6-31G(D)

Number of shells: 54 
Number of basis functions: 142

SCF model:

A restricted Hartree-Fock SCF calculation will be performed using Pulay DIIS extrapolation

Correlation model:

A MP2 calculation will be performed

Optimization:

$\begin{array}{ccrc}\text { Step } & \text { Energy } & \text { Max Grad. } & \text { Max Dist. } \\ 1 & -384.1681715 & 0.020342 & 0.088449 \\ 2 & -384.1711272 & 0.004524 & 0.092066 \\ 3 & -384.1713140 & 0.000739 & 0.034718 \\ 4 & -384.1713443 & 0.000546 & 0.023725 \\ 5 & -384.1713522 & 0.000137 & 0.007726 \\ 6 & -384.1713533 & 0.000104 & 0.003693 \\ 7 & -384.1713537 & 0.000086 & 0.002156\end{array}$

Dipole Moment (Debye) Tot $\quad 5.4920$

\begin{tabular}{|c|c|c|c|}
\hline \multicolumn{4}{|c|}{ Coordinates (Angstroms) } \\
\hline & $M \quad X$ & $\mathrm{Y}$ & $\mathrm{Z}$ \\
\hline 10 & -1.624413 & 0.413945 & 0.615323 \\
\hline $2 \mathrm{C}$ & -0.422899 & 0.118194 & -0.054849 \\
\hline $3 \mathrm{C}$ & 2.008158 & 0.681403 & -0.158072 \\
\hline $4 \mathrm{H}$ & 0.398241 & 2.092902 & 0.178357 \\
\hline $5 \mathrm{C}$ & 2.222389 & -0.767984 & -0.280722 \\
\hline $6 \mathrm{C}$ & -0.058183 & -1.310221 & 0.325726 \\
\hline $7 \mathrm{C}$ & 0.656390 & 1.065169 & 0.443166 \\
\hline $8 \mathrm{H}$ & 2.140413 & 1.071358 & -1.183232 \\
\hline $9 \mathrm{H}$ & -0.646172 & -2.0 & -0.165437 \\
\hline $10 \mathrm{C}$ & -2.8 & 0.2 & -0.1 \\
\hline $11 \mathrm{H}$ & -0.066300 & -1.447914 & 1.408047 \\
\hline $12 \mathrm{H}$ & 0.688639 & 1.008607 & 1.535784 \\
\hline $13 \mathrm{H}$ & -0.534363 & 0.202876 & -1.146991 \\
\hline $14 \mathrm{H}$ & & & 0.393974 \\
\hline 150 & 1.353266 & -1.662520 & -0.087181 \\
\hline $16 \mathrm{H}$ & -2.913414 & -0.837464 & -0.485414 \\
\hline $17 \mathrm{H}$ & -2.802980 & 0.857683 & -1.054630 \\
\hline $18 \mathrm{H}$ & -3.642172 & 0.474824 & 0.474387 \\
\hline $19 \mathrm{H}$ & 3.188518 & -1.177454 & -0.583933 \\
\hline
\end{tabular}

Energy is $\mathbf{- 3 8 4 . 1 7 1 3 5 3 6 5 3}$ hartrees 


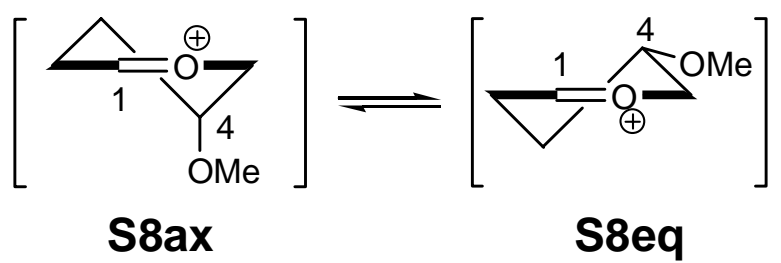

\begin{tabular}{|c|c|c|}
\hline Conformer & $\begin{array}{c}\text { rel } E(\mathrm{kcal} / \mathrm{mol}), \text { this work } \\
\mathrm{MP} 2 / 6-31 \mathrm{G}^{*}\end{array}$ & $\begin{array}{c}\text { rel } E(\mathrm{kcal} / \mathrm{mol}), \text { lit. }^{18} \\
\mathrm{HF} / 6-31 \mathrm{G}^{* *}\end{array}$ \\
\hline S8ax & 0 & 0 \\
\hline S8eq & 5.6 & 4.6 \\
\hline
\end{tabular}

- 4-Methyltetrahydropyrylium ion

Pseudoaxial 4-Methyltetrahydropyrylium ion, S9ax, MP2/6-31G* Equilibrium Geometry

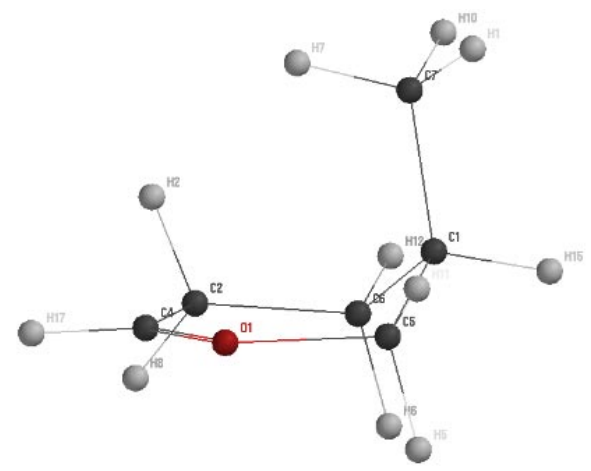

SPARTAN 'O2 Quantum Mechanics Program: (SGI/R10K)

Job type: Geometry optimization.

Method: RMP2(FC)

Basis set: 6-31G(D)

Number of shells: 50

Number of basis functions: 127

SCF model:

A restricted Hartree-Fock SCF calculation will be performed using Pulay DIIS extrapolation

Correlation model:

A MP2 calculation will be performed

Optimization:

\begin{tabular}{ccrr} 
Step & Energy & Max Grad. & \multicolumn{1}{c}{ Max Dist. } \\
1 & -309.1582957 & 0.020948 & 0.074141 \\
2 & -309.1610522 & 0.004677 & 0.073091
\end{tabular}




$\begin{array}{llll}3 & -309.1611973 & 0.000585 & 0.021367 \\ 4 & -309.1612120 & 0.000290 & 0.019108 \\ 5 & -309.1612147 & 0.000108 & 0.006304 \\ 6 & -309.1612152 & 0.000045 & 0.003166\end{array}$

Dipole Moment (Debye) Tot $\quad 3.2713$

\begin{tabular}{|c|c|c|c|}
\hline \multicolumn{4}{|c|}{ Coordinates (Angstroms) } \\
\hline & ATOM & $\mathrm{X} \quad \mathrm{Y}$ & $\mathrm{Z}$ \\
\hline $1 \mathrm{H}$ & $\mathrm{I} \quad-1.631614$ & 0.136547 & 1.302376 \\
\hline $2 \mathrm{C}$ & -0.886853 & 0.061334 & 0.499775 \\
\hline $3 \mathrm{C}$ & 1.507509 & 0.562620 & -0.066061 \\
\hline $4 \mathrm{H}$ & 0.209645 & 1.842911 & 1.083316 \\
\hline $5 \mathrm{C}$ & 1.502726 & -0.771958 & -0.682268 \\
\hline $6 \mathrm{C}$ & -0.591465 & -1.414995 & 0.365945 \\
\hline $7 \mathrm{C}$ & 0.392010 & 0.772958 & 0.956516 \\
\hline $8 \mathrm{H}$ & 455609 & 1.268511 & -0.912467 \\
\hline $9 \mathrm{H}$ & -1.359627 & -1.991912 & -0.148575 \\
\hline $0 \mathrm{H}$ & 2.296846 & -1.089556 & -1.362160 \\
\hline $11 \mathrm{H}$ & -0.309818 & -1.906904 & 1.299242 \\
\hline 12 & 0.709485 & 0.385446 & 1.930397 \\
\hline 13 & 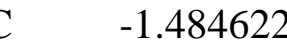 & 0.652024 & -0.778686 \\
\hline 14 & 2.514073 & 0.722452 & 0.348104 \\
\hline 15 & 0.615739 & -1.654637 & -0.522253 \\
\hline 16 & -2.435273 & 0.169733 & -1.020068 \\
\hline 17 & -0.827985 & 0.535718 & -1.646741 \\
\hline 18 & -1.676384 & 1.719707 & -0.646392 \\
\hline
\end{tabular}

Point Group: c1 Number of degrees of freedom: 48

\section{Energy is $-\mathbf{- 3 0 9 . 1 6 1 2 1 5 1 9 9}$}

Pseudoequatorial 4-Methyltetrahydropyrylium ion, S9eq, MP2/6-31G* Equilibrium Geometry

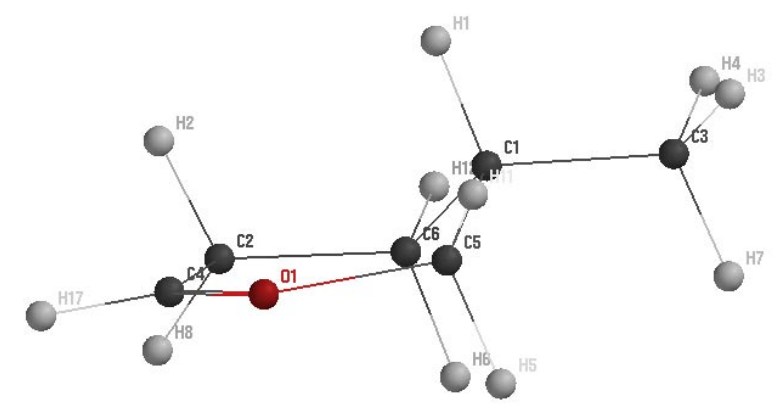

SPARTAN 'O2 Quantum Mechanics Program: (SGI/R10K)

Job type: Geometry optimization.

Method: RMP2(FC)

Basis set: $6-31 \mathrm{G}(\mathrm{D})$ 
Number of shells: 50

Number of basis functions: 127

SCF model:

A restricted Hartree-Fock SCF calculation will be performed using Pulay DIIS extrapolation

Correlation model:

A MP2 calculation will be performed

Optimization:

$\begin{array}{ccrr}\text { Step } & \text { Energy } & \text { Max Grad. } & \text { Max Dist. } \\ 1 & -309.1603237 & 0.011673 & 0.176562 \\ 2 & -309.1626583 & 0.003719 & 0.077842 \\ 3 & -309.1629214 & 0.001074 & 0.056188 \\ 4 & -309.1629558 & 0.000461 & 0.065394 \\ 5 & -309.1628912 & 0.001155 & 0.043915 \\ 6 & -309.1629749 & 0.000132 & 0.003978 \\ 7 & -309.1629751 & 0.000049 & 0.000874\end{array}$

Dipole Moment (Debye) Tot $\quad 4.2088$

\begin{tabular}{|c|c|c|c|}
\hline \multicolumn{4}{|c|}{ Coordinates (Angstroms) } \\
\hline & ATOM & $\mathrm{X} \quad \mathrm{Y}$ & $\mathrm{Z}$ \\
\hline $1 \mathrm{C}$ & -2.161408 & 0.285746 & -0.065066 \\
\hline $2 \mathrm{C}$ & -0.686816 & 0.008260 & -0.361921 \\
\hline $3 \mathrm{C}$ & 1.696413 & 0.717254 & -0.063636 \\
\hline $4 \mathrm{H}$ & -0.000815 & 2.045488 & -0.079046 \\
\hline $5 \mathrm{C}$ & 1.997355 & -0.722350 & -0.052227 \\
\hline $6 \mathrm{C}$ & -0.334760 & -1.362485 & 0.163416 \\
\hline $7 \mathrm{C}$ & 0.243095 & 1.040372 & 0.274807 \\
\hline $8 \mathrm{H}$ & 1.976012 & 1.057503 & -1.076537 \\
\hline $9 \mathrm{H}$ & -0.765766 & -2.191713 & -0.397132 \\
\hline $10 \mathrm{H}$ & 3.020416 & -1.092740 & -0.149887 \\
\hline $11 \mathrm{H}$ & -0.527963 & -1.487801 & 1.231475 \\
\hline $12 \mathrm{H}$ & 0.103820 & 1.041227 & 1.362065 \\
\hline $13 \mathrm{H}$ & -0.536538 & 0.010327 & -1.449929 \\
\hline $14 \mathrm{H}$ & & 1.208387 & 0.595477 \\
\hline $15 \mathrm{O}$ & 1.150227 & -1.651375 & 0.048530 \\
\hline $16 \mathrm{H}$ & -2.810653 & -0.470962 & -0.513643 \\
\hline $17 \mathrm{H}$ & -2.443217 & 1.256320 & -0.479742 \\
\hline $18 \mathrm{H}$ & -2.346046 & 0.308543 & 1.012996 \\
\hline
\end{tabular}

Point Group: c1 Number of degrees of freedom: 48

Energy is $\quad-\mathbf{3 0 9} .162975104$ 


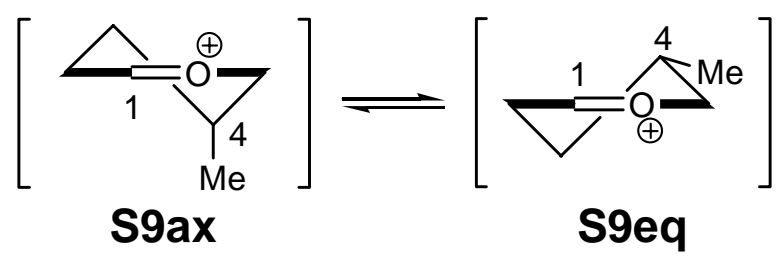

\begin{tabular}{|c|c|c|}
\hline Conformer & $\begin{array}{c}\mathrm{rel} E(\mathrm{kcal} / \mathrm{mol}), \text { this work } \\
\mathrm{MP} 2 / 6-31 \mathrm{G}^{*}\end{array}$ & $\begin{array}{c}\mathrm{rel} E(\mathrm{kcal} / \mathrm{mol}), \text { lit. }^{19} \\
\mathrm{HF} / 6-31 \mathrm{G}^{* *}\end{array}$ \\
\hline S9ax & 1.1 & 0.73 \\
\hline S9eq & 0 & 0 \\
\hline
\end{tabular}

- 1-Ethoxy-4-Methoxytetrahydropyrylium ion

Pseudoaxial 1-Ethoxy-4-Methoxytetrahydropyrylium ion, 4-OMe, MP2/6-31G* Equilibrium Geometry

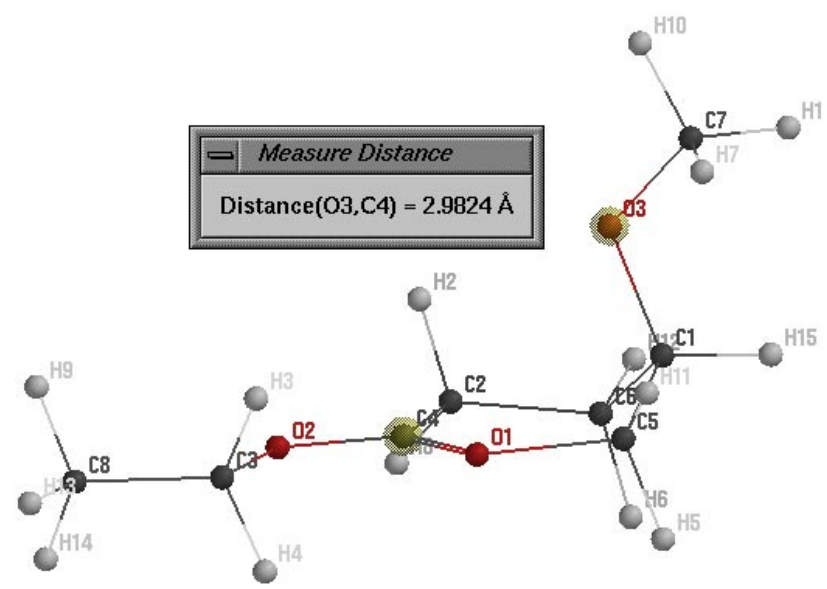

SPARTAN 'O2 Quantum Mechanics Program: (SGI/R10K)

Job type: Geometry optimization.

Method: RMP2(FC)

Basis set: 6-31G(D)

Number of shells: 74

Number of basis functions: 195

SCF model:

A restricted Hartree-Fock SCF calculation will be performed using Pulay DIIS extrapolation

Correlation model:

A MP2 calculation will be performed

Optimization:

$\begin{array}{ccrrr}\text { Step } & \text { Energy } & \text { Max Grad. } & \text { Max Dist. } & \\ 1 & -537.5595152 & 0.064471 & 0.107745 & 1\end{array}$




$\begin{array}{ccccc}2 & -537.5797699 & 0.036568 & 0.157861 & 1 \\ 3 & -537.5871379 & 0.021102 & 0.141310 & 1 \\ 4 & -537.5857703 & 0.021490 & 0.165872 & \\ 5 & -537.5903113 & 0.004528 & 0.161485 & \\ 6 & -537.5914501 & 0.002732 & 0.030174 & \\ 7 & -537.5915156 & 0.001495 & 0.016865 & \\ 8 & -537.5915288 & 0.000752 & 0.018108 & \\ 9 & -537.5915328 & 0.000093 & 0.003231 & \\ 10 & -537.5915331 & 0.000045 & 0.002120 & \end{array}$

Dipole Moment (Debye) Tot 2.0386

\begin{tabular}{rrrrrr}
\multicolumn{5}{c}{ Coordinates (Angstroms) } \\
1 & O & -3.177299 & 0.111457 & 0.486370 \\
2 & $\mathrm{C}$ & -1.880829 & 0.193717 & -0.059802 \\
3 & $\mathrm{C}$ & 0.148800 & 1.623957 & -0.217075 \\
4 & $\mathrm{H}$ & -1.886910 & 2.343650 & -0.102111 \\
5 & $\mathrm{C}$ & 0.925321 & 0.371516 & 0.017173 \\
6 & $\mathrm{C}$ & -1.056466 & -0.916881 & 0.578204 \\
7 & $\mathrm{C}$ & -1.280425 & 1.536497 & 0.315405 \\
8 & $\mathrm{H}$ & 0.158417 & 1.779409 & -1.305329 \\
9 & $\mathrm{H}$ & -1.275542 & -1.913779 & 0.198307 \\
10 & $\mathrm{C}$ & -4.087108 & -0.696772 & -0.277676 \\
11 & $\mathrm{H}$ & -1.163453 & -0.891493 & 1.663697 \\
12 & $\mathrm{H}$ & -1.303270 & 1.641772 & 1.405175 \\
13 & $\mathrm{H}$ & -1.897349 & 0.077283 & -1.155117 \\
14 & $\mathrm{H}$ & 0.718904 & 2.454301 & 0.211291 \\
15 & $\mathrm{O}$ & 0.409358 & -0.769679 & 0.319300 \\
16 & $\mathrm{H}$ & -3.753196 & -1.737953 & -0.341988 \\
17 & $\mathrm{H}$ & -4.212850 & -0.287187 & -1.285400 \\
18 & $\mathrm{H}$ & -5.034863 & -0.655683 & 0.256082 \\
19 & $\mathrm{O}$ & 2.196926 & 0.433432 & -0.136887 \\
20 & $\mathrm{C}$ & 3.032142 & -0.798426 & 0.036420 \\
21 & $\mathrm{C}$ & 4.455780 & -0.371645 & -0.198981 \\
22 & $\mathrm{H}$ & 2.667012 & -1.525150 & -0.690613 \\
23 & $\mathrm{H}$ & 2.845252 & -1.160142 & 1.048325 \\
24 & $\mathrm{H}$ & 4.763367 & 0.384009 & 0.525249 \\
25 & $\mathrm{H}$ & 4.586386 & 0.019431 & -1.208987 \\
26 & $\mathrm{H}$ & 5.101896 & -1.245642 & -0.081030
\end{tabular}

Point Group: c1 Number of degrees of freedom: 72

Energy is $\mathbf{- 5 3 7 . 5 8 3 1 2 0 1 4 7}$ hartrees 
Pseudoequatorial 1-Ethoxy-4-Methoxytetrahydropyrylium ion, 3-OMe, MP2/6-31G* Equilibrium Geometry

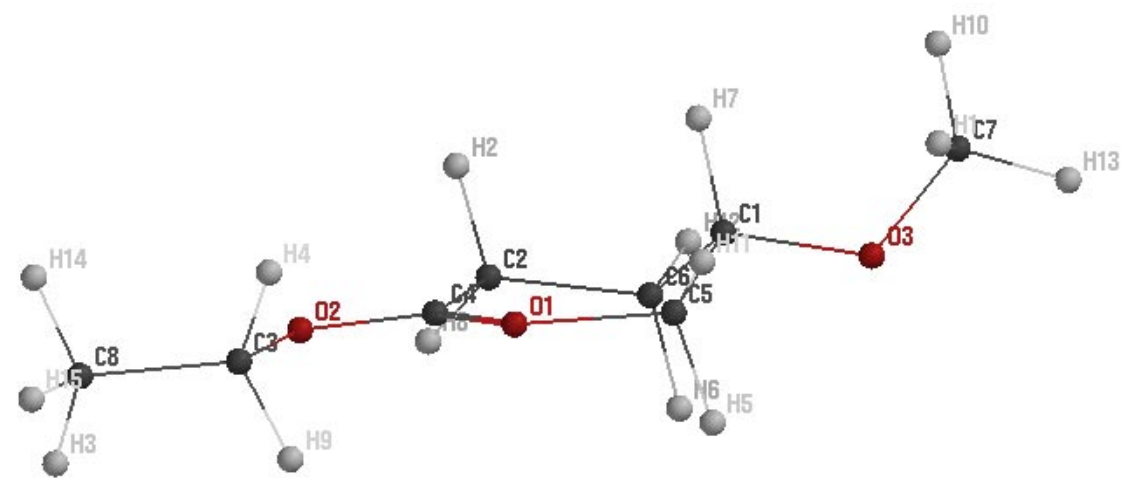

SPARTAN 'O2 Quantum Mechanics Program: (SGI/R10K)

Job type: Geometry optimization.

Method: RMP2(FC)

Basis set: 6-31G(D)

Number of shells: 74

Number of basis functions: 195

SCF model:

A restricted Hartree-Fock SCF calculation will be performed using Pulay DIIS extrapolation

Correlation model:

A MP2 calculation will be performed

Optimization:

$\begin{array}{ccrrr}\text { Step } & \text { Energy } & \text { Max Grad. } & \text { Max Dist. } & \\ 1 & -537.5427319 & 0.043933 & 0.158465 & 1 \\ 2 & -537.5579442 & 0.026800 & 0.195895 & 1 \\ 3 & -537.5670603 & 0.016798 & 0.133868 & 1 \\ 4 & -537.5612330 & 0.049677 & 0.188857 & \\ 5 & -537.5720508 & 0.021014 & 0.194294 & \\ 6 & -537.5778561 & 0.013759 & 0.187023 & \\ 7 & -537.5815683 & 0.005199 & 0.125370 & \\ 8 & -537.5828139 & 0.001990 & 0.050747 & \\ 9 & -537.5829860 & 0.001015 & 0.060520 & \\ 10 & -537.5830790 & 0.001261 & 0.053522 \\ 11 & -537.5831098 & 0.000523 & 0.016249 \\ 12 & -537.5831181 & 0.000195 & 0.006421 \\ 13 & -537.5831197 & 0.000177 & 0.005109 \\ 14 & -537.5831201 & 0.000064 & 0.001255 & \end{array}$

Dipole Moment (Debye) $\quad$ Tot 2.3548 


\begin{tabular}{|c|c|c|c|}
\hline \multicolumn{4}{|c|}{ Coordinates (Angstroms) } \\
\hline & ATOM & $\mathrm{X} \quad \mathrm{Y}$ & $\mathrm{Z}$ \\
\hline $1 \mathrm{H}$ & -3.051486 & 0.406989 & 0.848079 \\
\hline $2 \mathrm{C}$ & -2.044628 & 0.338857 & 0.409164 \\
\hline $3 \mathrm{C}$ & -0.188613 & 1.518773 & -0.705349 \\
\hline $4 \mathrm{H}$ & -2.240020 & 2.170998 & -0.706824 \\
\hline $5 \mathrm{C}$ & 0.675171 & 0.535254 & 0.004681 \\
\hline $6 \mathrm{C}$ & -1.1 & -0.2410 & 712 \\
\hline $7 \mathrm{C}$ & -1.5 & & \\
\hline $8 \mathrm{H}$ & -0.3 & 1.11 & -1.71 \\
\hline $9 \mathrm{H}$ & -1.2 & -1.2 & 78 \\
\hline $10 \mathrm{C}$ & -2.9 & -1.5 & -0.713509 \\
\hline $1 \mathrm{H}$ & $-1 .($ & 883 & 190 \\
\hline $12 \mathrm{H}$ & -1.4 & 2.36 & 0.85 \\
\hline $13 \mathrm{O}$ & -2.0 & -0.4 & -0.7 \\
\hline $14 \mathrm{H}$ & & 2.4 & -0.81 \\
\hline & & -0.2 & 0.96 \\
\hline $16 \mathrm{H}$ & -3.9 & -1.226608 & -0.586868 \\
\hline $17 \mathrm{H}$ & -2.6 & -2.278361 & 0.091022 \\
\hline $18 \mathrm{H}$ & -2.8 & -2.0 & -1.6 \\
\hline $19 \mathrm{O}$ & & 0.442311 & -0.38 \\
\hline $20 \mathrm{C}$ & 2.80 & -0.55 & 0.249926 \\
\hline $21 \mathrm{H}$ & 2.34 & -1.53 & 0.107806 \\
\hline $22 \mathrm{H}$ & 760 & -0.316093 & 1.313012 \\
\hline $23 \mathrm{C}$ & 4.13 & -0.410025 & -0.447426 \\
\hline $24 \mathrm{H}$ & & -0.621704 & -1.513614 \\
\hline & 4.835072 & -1.126844 & -0.012644 \\
\hline $26 \mathrm{H}$ & 4.537963 & 0.593970 & -0.311378 \\
\hline
\end{tabular}

Point Group: c1 Number of degrees of freedom: 72

Energy is $\mathbf{- 5 3 7 . 5 9 1 5 3 3 1 1 3 ~ h a r t r e e s ~}$

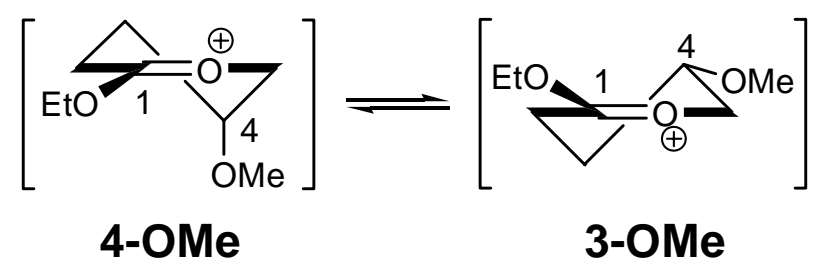

$\mathrm{MP} 2 / 6-31 \mathrm{G}^{*} E \mathrm{rel}(\mathrm{kcal} / \mathrm{mol})=\mathbf{0}$

5.3 
- 1-Ethoxy-4-Methyltetrahydropyrylium ion

Pseudoaxial 1-Ethoxy-4-Methyltetrahydropyrylium ion, 4-Me, MP2/6-31G* Equilibrium Geometry

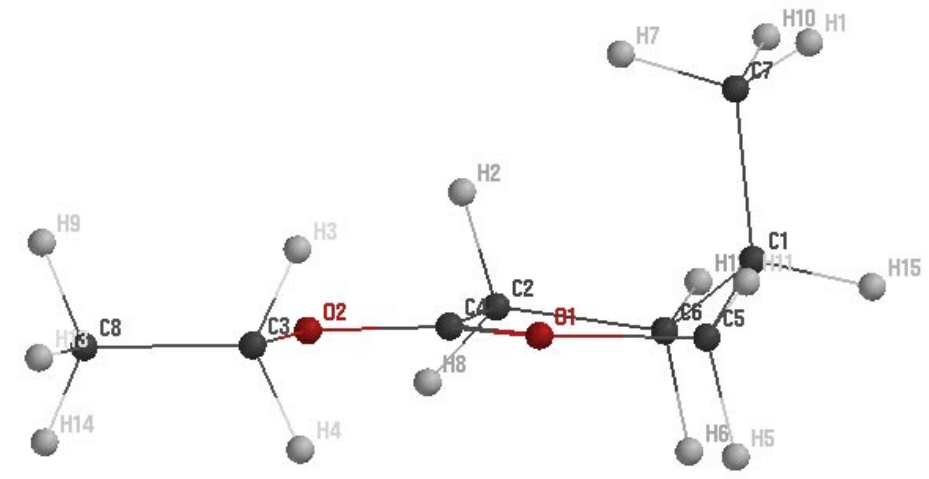

SPARTAN 'O2 Quantum Mechanics Program: (SGI/R10K)

Job type: Geometry optimization.

Method: RMP2(FC)

Basis set: 6-31G(D)

Number of shells: 70

Number of basis functions: 180

SCF model:

A restricted Hartree-Fock SCF calculation will be performed using Pulay DIIS extrapolation

Correlation model:

A MP2 calculation will be performed

Optimization:

$\begin{array}{ccccc}\text { Step } & \text { Energy } & \text { Max Grad. } & \text { Max Dist. } & \\ 1 & -462.5728912 & 0.000032 & 0.127715 & 1 \\ 2 & -462.5726975 & 0.001108 & 0.115816 & \\ 3 & -462.5728902 & 0.000044 & 0.011560 & \\ 4 & -462.5728916 & 0.000050 & 0.001107\end{array}$

Dipole Moment (Debye) Tot $\quad 1.3371$

\begin{tabular}{|c|c|c|c|}
\hline \multicolumn{4}{|c|}{ Coordinates (Angstroms) } \\
\hline & ATOM & $\mathrm{X} \quad \mathrm{Y}$ & $\mathrm{Z}$ \\
\hline $1 \mathrm{H}$ & -3.266831 & -0.503427 & 0.710018 \\
\hline $2 \mathrm{C}$ & -2.299676 & -0.289749 & 0.236775 \\
\hline $3 \mathrm{C}$ & -0.518549 & 1.465287 & 0.009964 \\
\hline $\mathrm{H}$ & -2.631137 & 1.854372 & 0.126293 \\
\hline $5 \mathrm{C}$ & 0.436567 & 0.336371 & 0.202998 \\
\hline $6 \mathrm{C}$ & -1.307361 & -1.206836 & 0.915105 \\
\hline
\end{tabular}




\begin{tabular}{|c|c|c|c|}
\hline $7 \mathrm{C}$ & -1.911141 & 1.155222 & 0.559932 \\
\hline $8 \mathrm{H}$ & -0.540035 & 1.656373 & -1.070799 \\
\hline $9 \mathrm{H}$ & -1.374864 & -2.247904 & 0.599679 \\
\hline $10 \mathrm{O}$ & 1.674359 & 0.571696 & -0.043623 \\
\hline $11 \mathrm{H}$ & -1.331856 & -1.143512 & 2.004883 \\
\hline $12 \mathrm{H}$ & -1.926446 & 1.313344 & 1.644069 \\
\hline $13 \mathrm{C}$ & -2.417807 & -0.566780 & -1.262774 \\
\hline $14 \mathrm{H}$ & -0.070085 & 2.356797 & 0.461032 \\
\hline $15 \mathrm{O}$ & 0.109876 & -0.855777 & 0.560438 \\
\hline $16 \mathrm{H}$ & -2.793936 & -1.577937 & -1.438982 \\
\hline $17 \mathrm{H}$ & -1.459597 & -0.477953 & -1.783866 \\
\hline $18 \mathrm{H}$ & -3.116930 & 0.134786 & -1.725208 \\
\hline $19 \mathrm{C}$ & 2.682720 & -0.526930 & 0.087767 \\
\hline $20 \mathrm{H}$ & 2.370823 & -1.318911 & -0.594651 \\
\hline $21 \mathrm{H}$ & 2.624587 & -0.881747 & 1.117596 \\
\hline $22 \mathrm{C}$ & 4.011822 & 0.084532 & -0.264937 \\
\hline $23 \mathrm{H}$ & 4.010916 & 0.460685 & -1.288989 \\
\hline $24 \mathrm{H}$ & 4.780710 & -0.688188 & -0.181777 \\
\hline $25 \mathrm{H}$ & 4.263871 & 0.896186 & 0.419056 \\
\hline
\end{tabular}

\section{Energy is $-\mathbf{4 6 2 . 5 7 2 8 9 1 6 1 0}$ hartrees}

Pseudoequatorial 1-Ethoxy-4-Methyltetrahydropyrylium ion, 3-Me, MP2/6-31G* Equilibrium Geometry

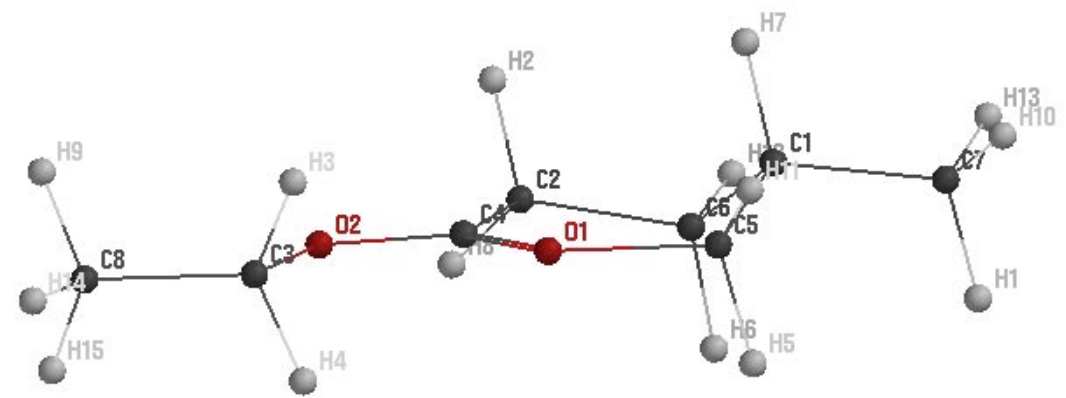

SPARTAN 'O2 Quantum Mechanics Program: (SGI/R10K)

Job type: Geometry optimization.

Method: RMP2(FC)

Basis set: 6-31G(D)

Number of shells: 70

Number of basis functions: 180

SCF model:

A restricted Hartree-Fock SCF calculation will be performed using Pulay DIIS extrapolation

Correlation model:

A MP2 calculation will be performed 
Optimization:

$\begin{array}{ccrr}\text { Step } & \text { Energy } & \text { Max Grad. } & \text { Max Dist. } \\ 1 & -462.5499620 & 0.077582 & 0.142575 \\ 2 & -462.5711379 & 0.010020 & 0.089708 \\ 3 & -462.5740261 & 0.005016 & 0.159891 \\ 4 & -462.5744242 & 0.001663 & 0.063308 \\ 5 & -462.5744955 & 0.000280 & 0.029102 \\ 6 & -462.5744986 & 0.000114 & 0.014672 \\ 7 & -462.5744996 & 0.000077 & 0.000927\end{array}$

Dipole Moment (Debye) Tot 1.0076

\begin{tabular}{|c|c|c|c|}
\hline \multicolumn{4}{|c|}{ Coordinates (Angstroms) } \\
\hline & ATOM & $X \quad Y$ & $\mathrm{Z}$ \\
\hline $1 \mathrm{C}$ & -3.546667 & -0.507365 & -0.089495 \\
\hline $2 \mathrm{C}$ & -2.088205 & -0.142197 & -0.368231 \\
\hline $3 \mathrm{C}$ & -0.237082 & 1.512668 & -0.098590 \\
\hline $4 \mathrm{H}$ & -2.333985 & 1.989709 & -0.052802 \\
\hline $5 \mathrm{C}$ & 0.660591 & 0.326583 & 0.006 \\
\hline $6 \mathrm{C}$ & -1.197507 & -1.239723 & 0.165309 \\
\hline $7 \mathrm{C}$ & -1.67 & 489 & $0.27^{\prime}$ \\
\hline $8 \mathrm{H}$ & -0.170411 & 1.847147 & -1.143385 \\
\hline $9 \mathrm{H}$ & -1.2 & -2.16 & -0.402312 \\
\hline $10 \mathrm{O}$ & 1.925042 & 0.54 & -0.021948 \\
\hline $11 \mathrm{H}$ & -1.3 & 0568 & 1.22 \\
\hline $12 \mathrm{H}$ & -1.782461 & 1.107541 & 1.367083 \\
\hline $13 \mathrm{H}$ & -1.94 & -0.0 & -1.454406 \\
\hline $14 \mathrm{H}$ & 0.1 & 2.3 & 0.506 \\
\hline $15 \mathrm{O}$ & 1697 & -0.89 & 0.090341 \\
\hline $16 \mathrm{H}$ & -3.73 & -0.563750 & 0.986503 \\
\hline $17 \mathrm{H}$ & -3.8 & -1.467730 & -0.539537 \\
\hline $18 \mathrm{H}$ & -4.206178 & 0.254180 & -0.511989 \\
\hline $19 \mathrm{C}$ & 2.882368 & -0.604304 & 0.033257 \\
\hline $20 \mathrm{H}$ & 2.651465 & -1.242729 & -0.820670 \\
\hline $21 \mathrm{H}$ & 2.674548 & -1.138226 & 0.961455 \\
\hline & 4.260076 & -0.001012 & -0.019783 \\
\hline $23 \mathrm{H}$ & 4.408976 & 0.555708 & -0.946092 \\
\hline & 4.993722 & -0.810682 & 0.018028 \\
\hline $25 \mathrm{H}$ & 4.431775 & 0.659272 & 0.831513 \\
\hline
\end{tabular}

Point Group: c1 Number of degrees of freedom: 69

Energy is $\mathbf{- 4 6 2 . 5 7 4 4 9 9 6 0 3}$ hartrees 


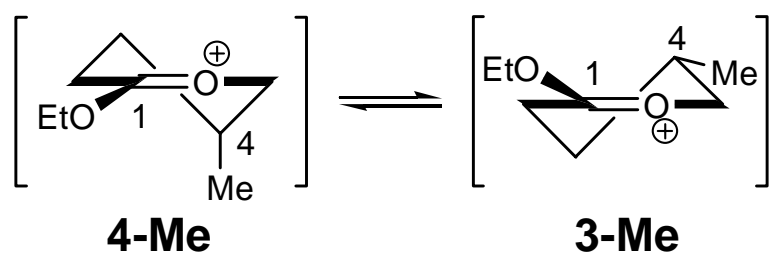

$\mathrm{MP} 2 / 6-31 \mathrm{G}^{*} E \mathrm{rel}(\mathrm{kcal} / \mathrm{mol})=\mathbf{1 . 0 0}$

\section{Bibliography}

(1) Pangborn, A. B.; Giardello, M. H.; Grubbs, R. H.; Rosen, R. K.; Timmers, F. J. Organometallics 1996, 15, 1518-1520.

(2) Brown, H. C.; Vara Prasad, J. V. N.; Zee, S.-H. J. Org. Chem. 1985, 50, 1582-1589.

(3) Determined by integration of the $\mathrm{C} 1$-methine for each anomer using single-scan ${ }^{1} \mathrm{H}$ NMR.

(4) Romero, J. A. C.; Tabacco, S. A.; Woerpel, K. A. J. Am. Chem. Soc. 2000, 122, 168-169.

(5) Misske, A. M.; Hoffmann, H. M. R. Tetrahedron 1999, 55, 4315-4324.

(6) Suzuki, T.; Usui, K.; Miyake, Y.; Namikoshi, M.; Nakada, M. Org. Lett. 2004, 6, 553556.

(7) Srivastava, R. M.; Brown, R. K. Can. J. Chem. 1970, 48, 2334-2340.

(8) Prepared in alternate fashion by Srivastava, R. M.; Brown, R. K. Can. J. Chem. 1970, 48, 2334-2340; however, only elemental analysis and boiling point data were provided.

(9) Wiberg, K. B.; Waldron, R. F. J. Am. Chem. Soc. 1991, 113, 7705-7709.

(10) Childs, R. F.; Kostyk, M. D.; Lock, C. J. L.; Mahendran, M. Can. J. Chem. 1991, 69, 2024-2032.

(11) Beaulieu, N.; Deslongchamps, P. Can. J. Chem. 1980, 58, 164-167.

(12) Haasnoot, C. A. G.; de Leeuw, F. A. A. M.; Altona, C. Tetrahedron 1980, 36, 2783-2792.

(13) Kozikowski, A. P.; Ghosh, A. K. J. Org. Chem. 1985, 50, 3017-3019.

(14) Boudreault, N.; Ball, R. G.; Bayly, C.; Bernstein, M. A.; Leblanc, Y. Tetrahedron 1994, 50, 7947-7956.

(15) SPARTAN '02 Wavefunction Developers:

B.J. Deppmeier, A.J. Driessen, T.S. Hehre, W.J. Hehre, J.A. Johnson, P.E. Klunzinger, J.M.

Leonard, I.N. Pham, W.J. Pietro, Jianguo Yu

Q-Chem Developers:

J. Kong, C.A. White, A.I. Krylov, C.D. Sherrill, R.D. Adamson, T.R. Furlani, M.S. Lee, A.M.

Lee, S.R. Gwaltney, T.R. Adams, C. Ochsenfeld, A.T.B. Gilbert, G.S. Kedziora, V.A.

Rassolov, D. R. Maurice, N. Nair, Y. Shao, N.A. Besley, P.E. Maslen, J.P. Dombroski, H.

Dachsel, W.M. Zhang, P.P. Korambath, J. Baker, E.F. C. Byrd, T. Van Voorhis, M. Oumi, S. Hirata, C.P. Hsu, N. Ishikawa, J. Florian, A. Warshel, B.G. Johnson, P.M.W. Gill, M. Head-

Gordon, J.A. Pople.

(16) Evans, D. A.; Black, W. C. J. Am. Chem. Soc. 1993, 115, 4497-4513.

(17) Burt, R. A.; Chiang, Y.; Hall, J., H. K.; Kresge, A. J. J. Am. Chem. Soc. 1982, 104, 36873690.

(18) Miljkovic, M.; Yeagley, D.; Deslongchamps, P.; Dory, Y. L. J. Org. Chem. 1997, 62, 7597-7604.

(19) Woods, R. J.; Andrews, C. W.; Bowen, J. P. J. Am. Chem. Soc. 1992, 114, 859-864. 
VI. Analytical Data

${ }^{1} \mathrm{H},{ }^{13} \mathrm{C}$ NMR Spectra of Relevant Compounds and of 5, 6, and 9
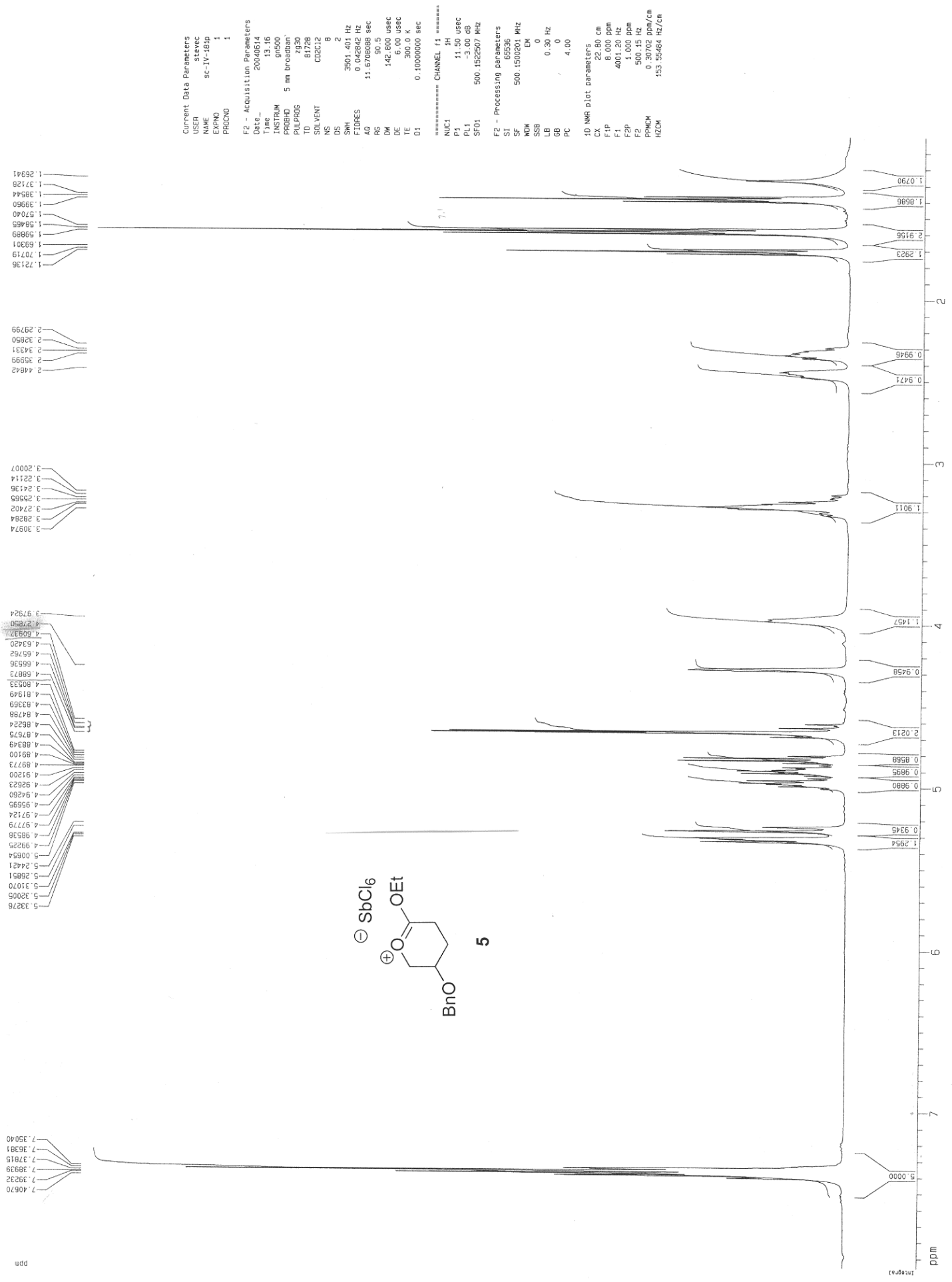

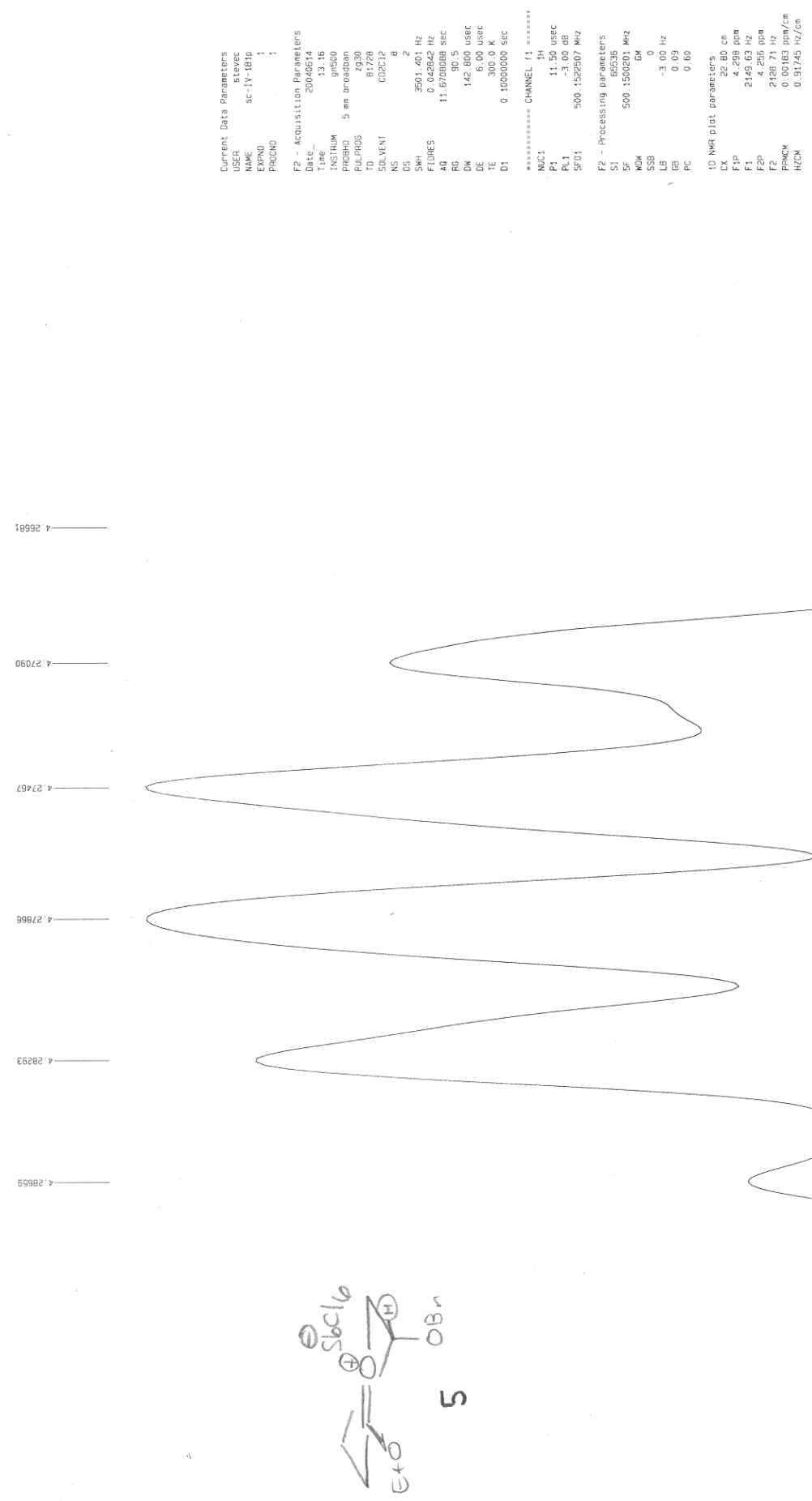

wo

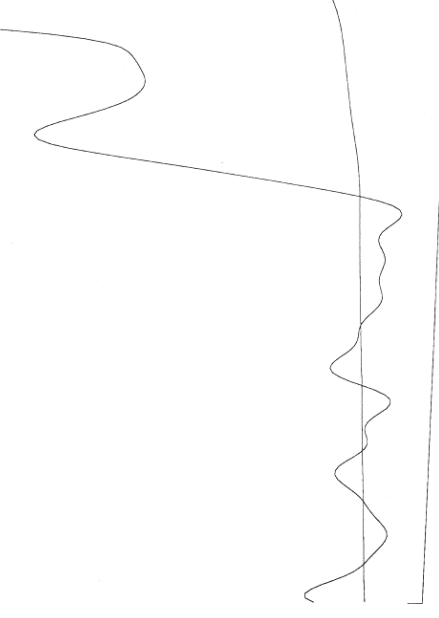



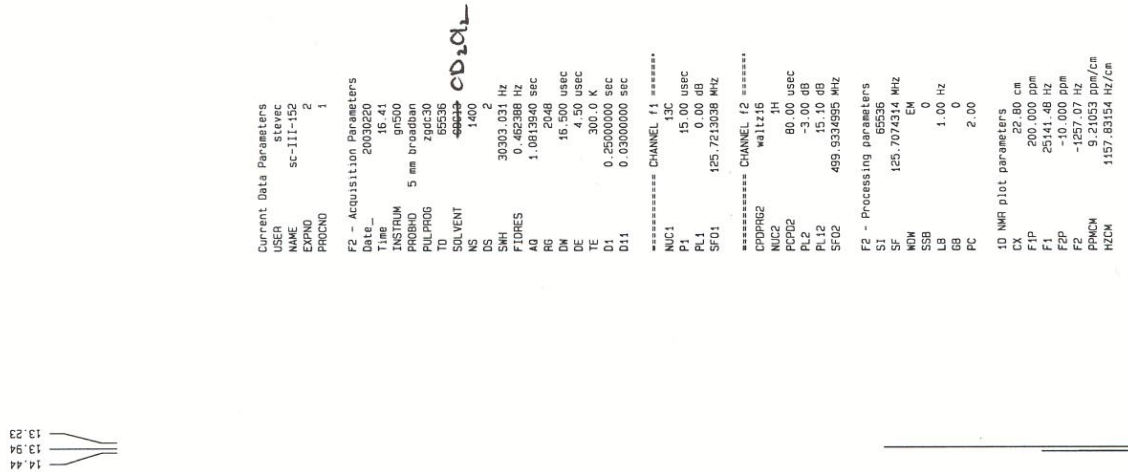

Sp:t2

$p 9.52$

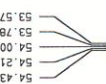

ร2: $\angle 9$

${ }_{06}^{92 \cdot 0 L}=$

${ }_{58} \mathrm{~S}^{\mathrm{S}} \mathrm{L}-$

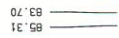

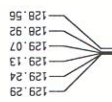

$266^{9} 9 \mathrm{~L}$

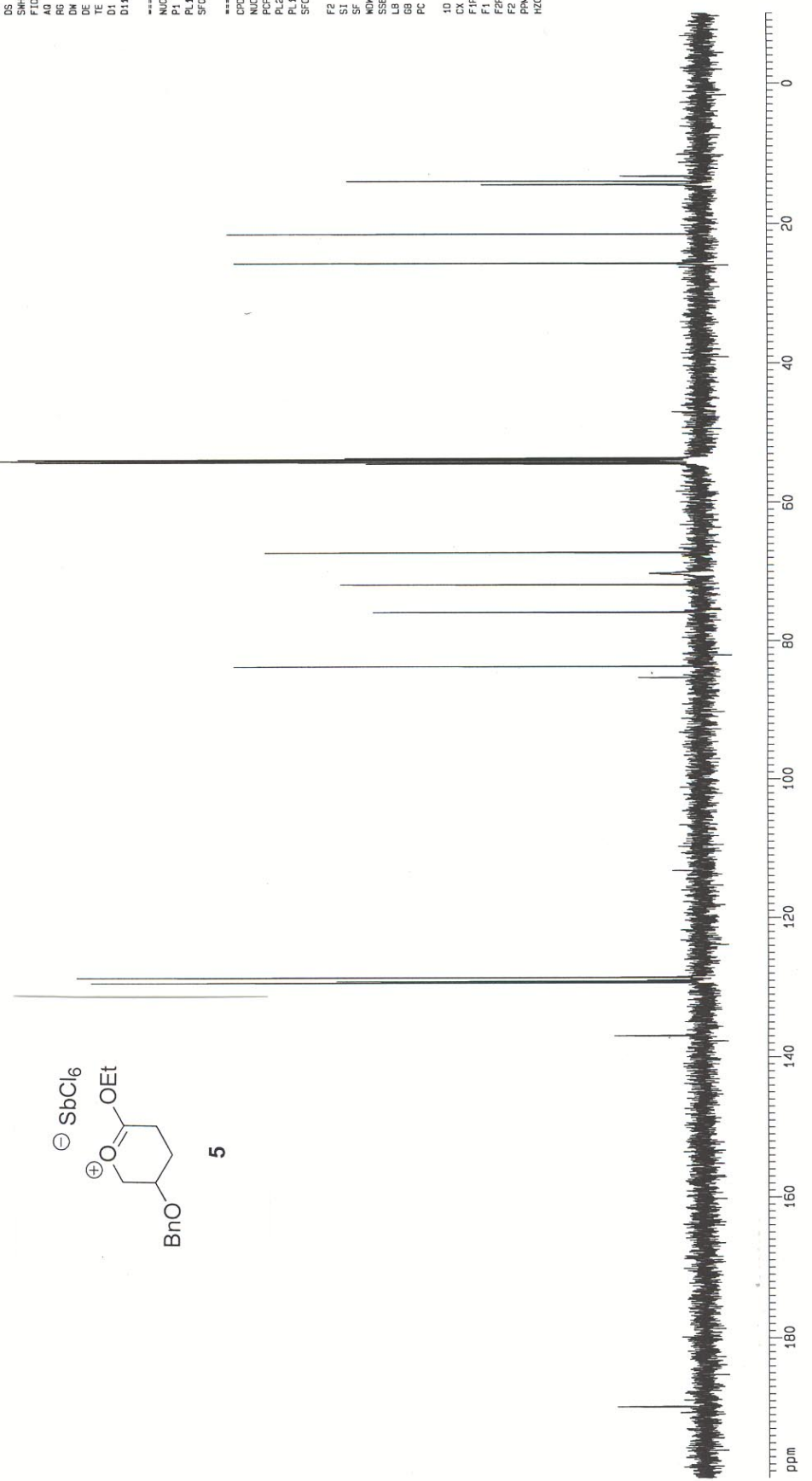

then 


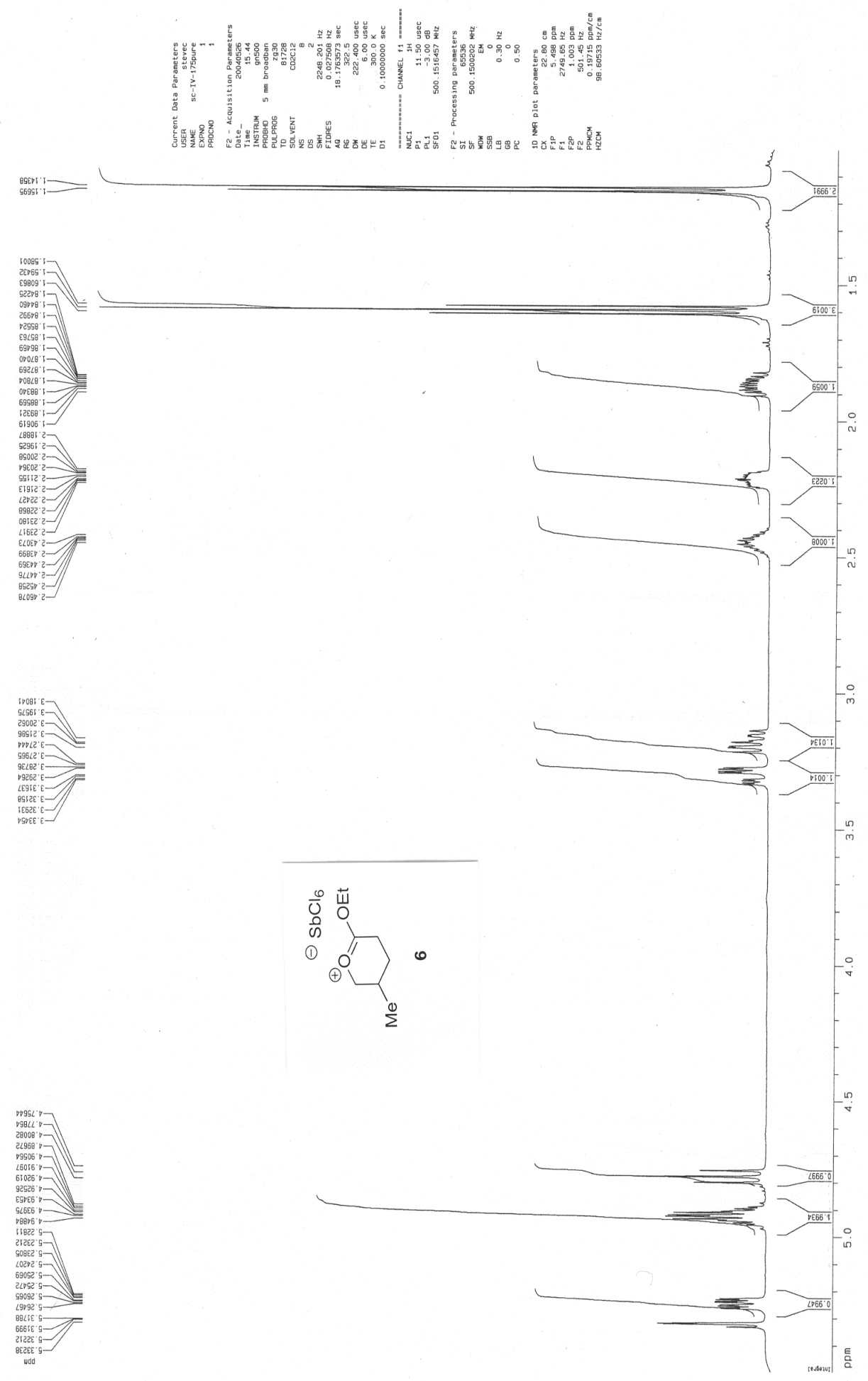




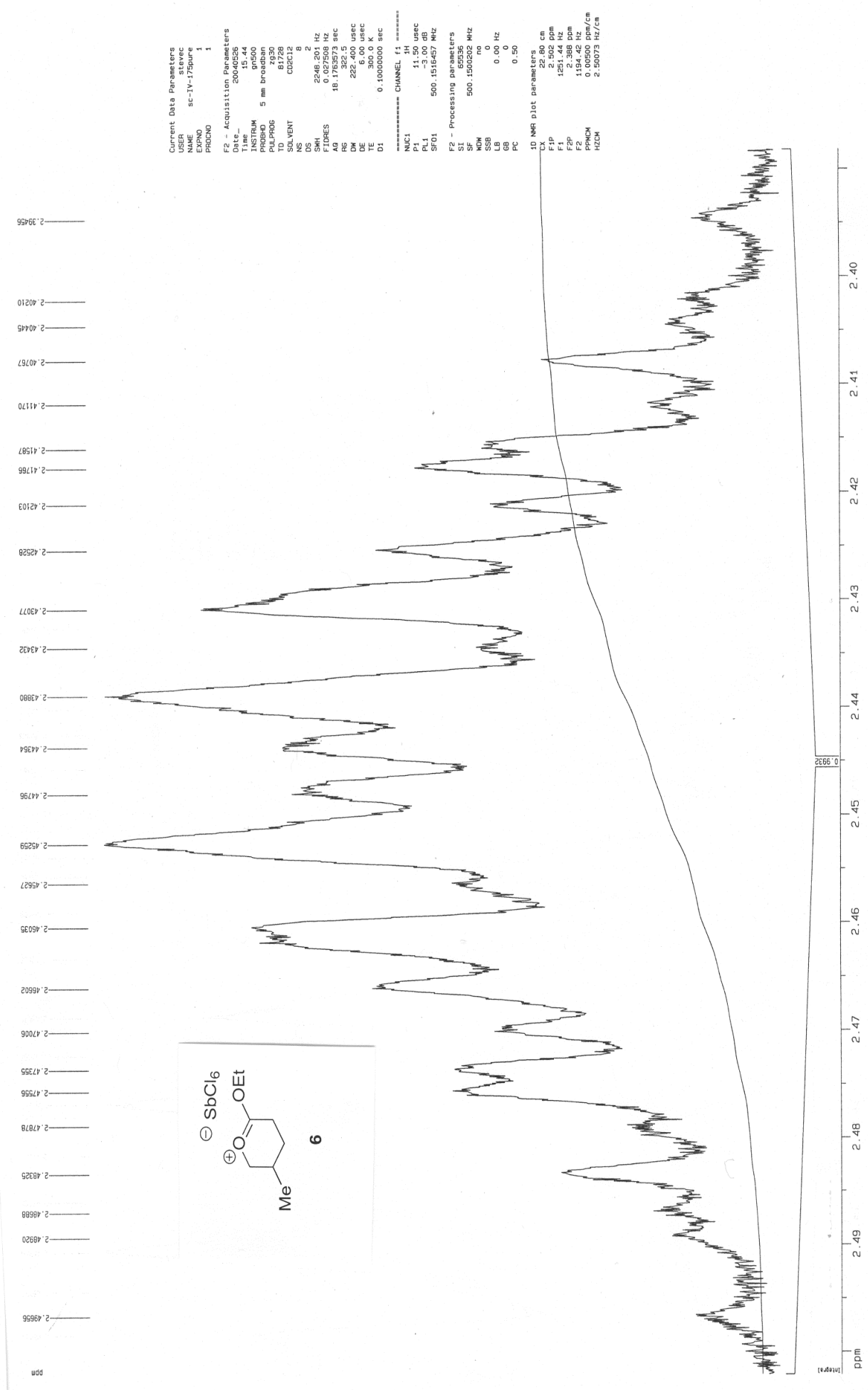




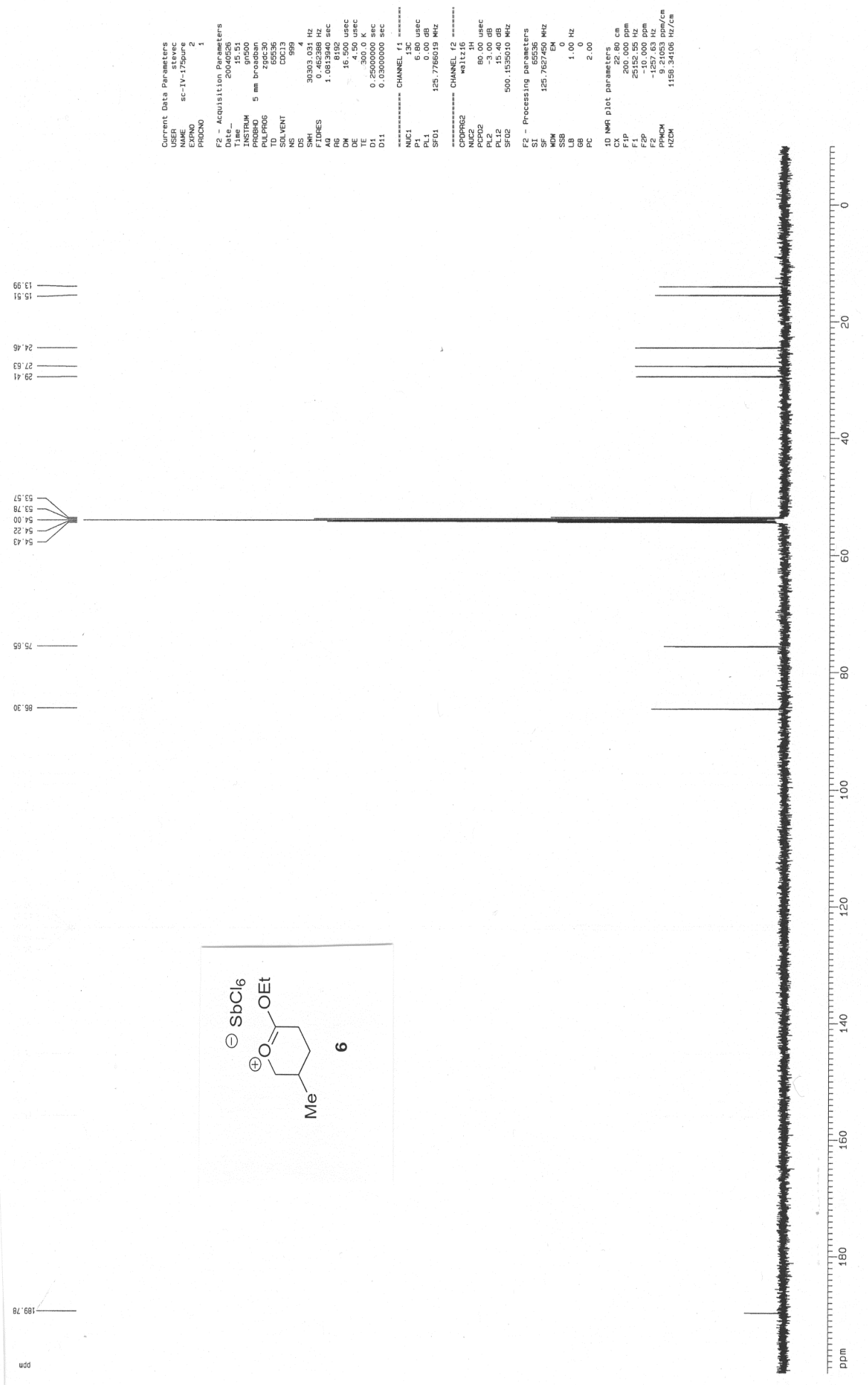



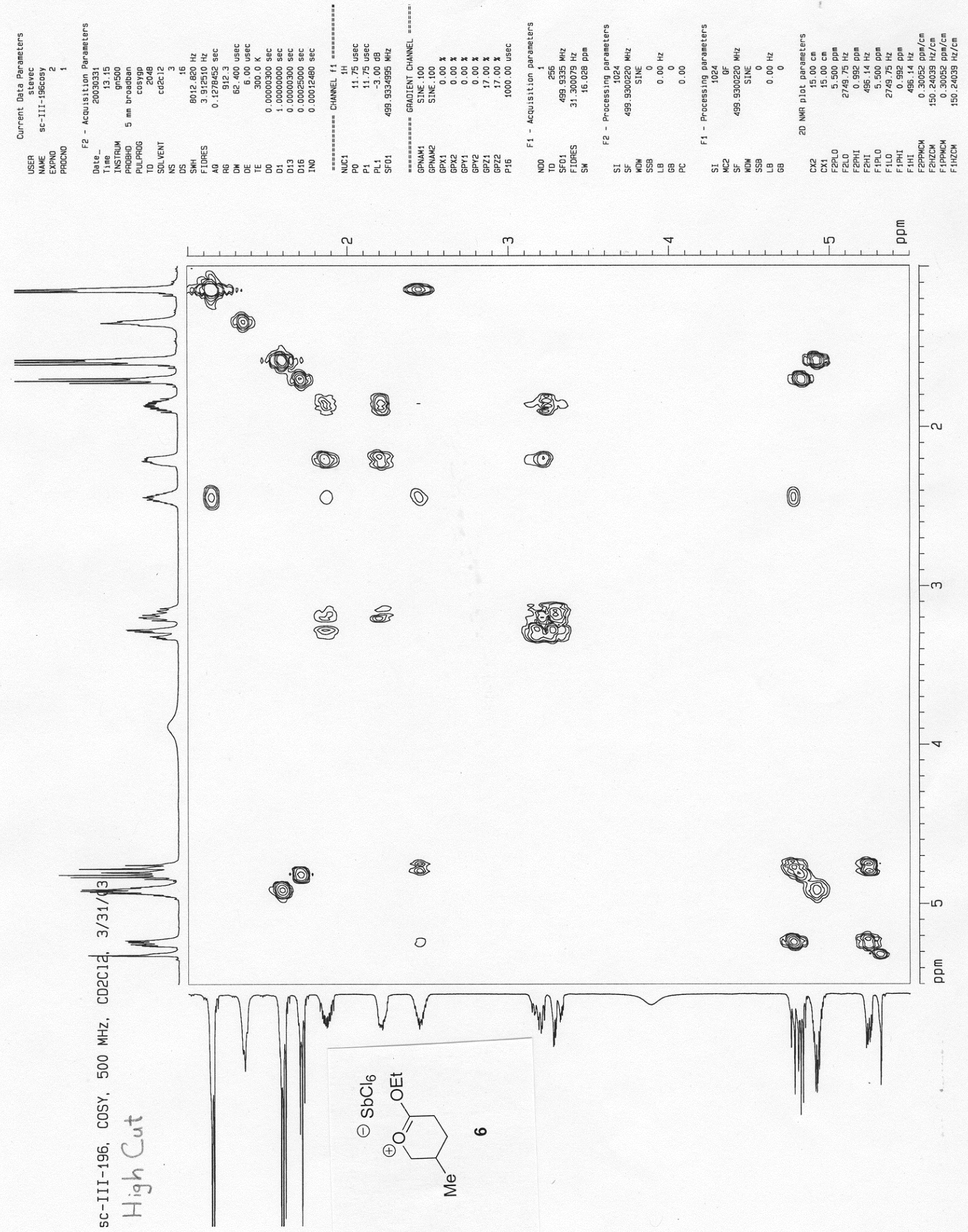

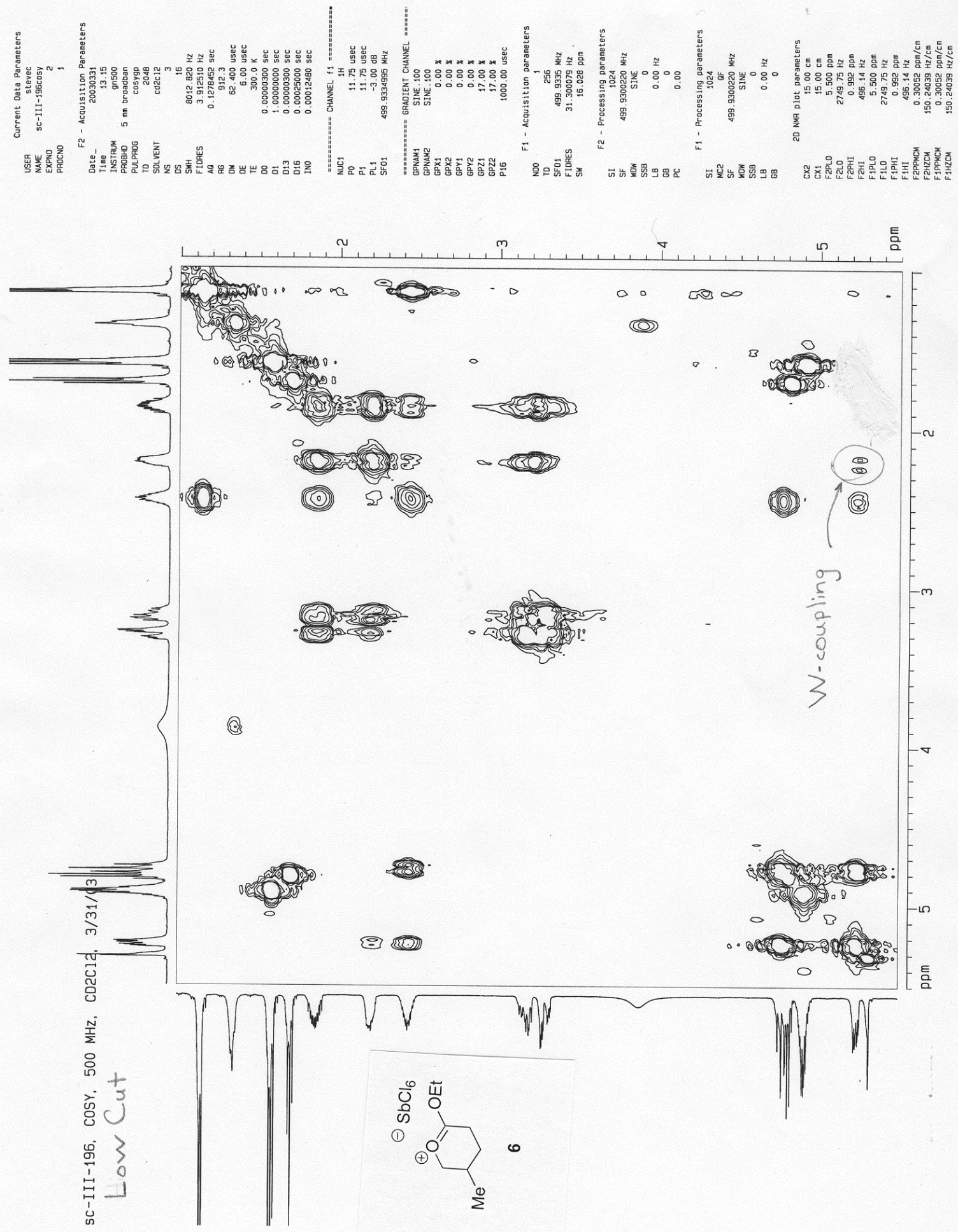

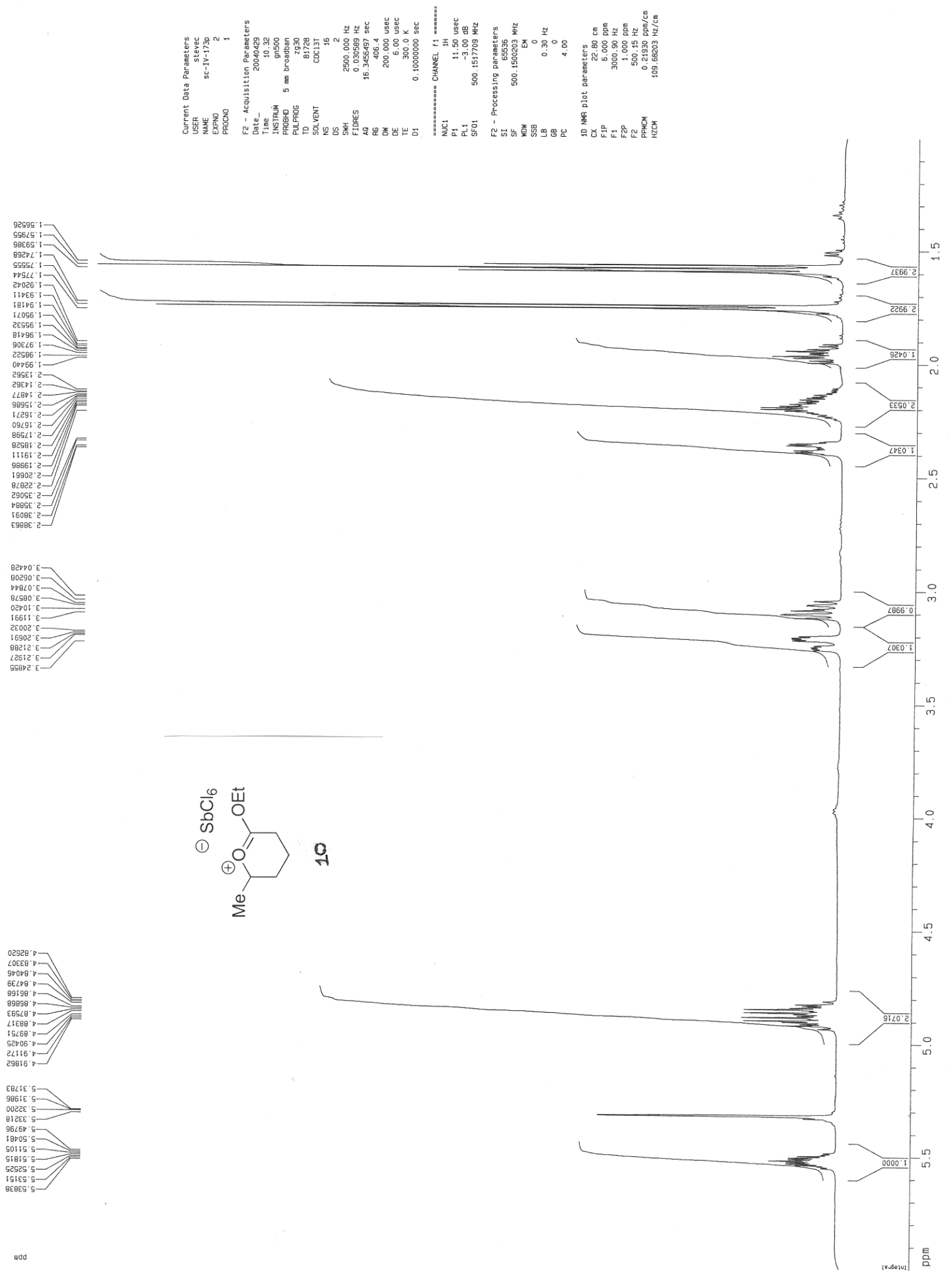

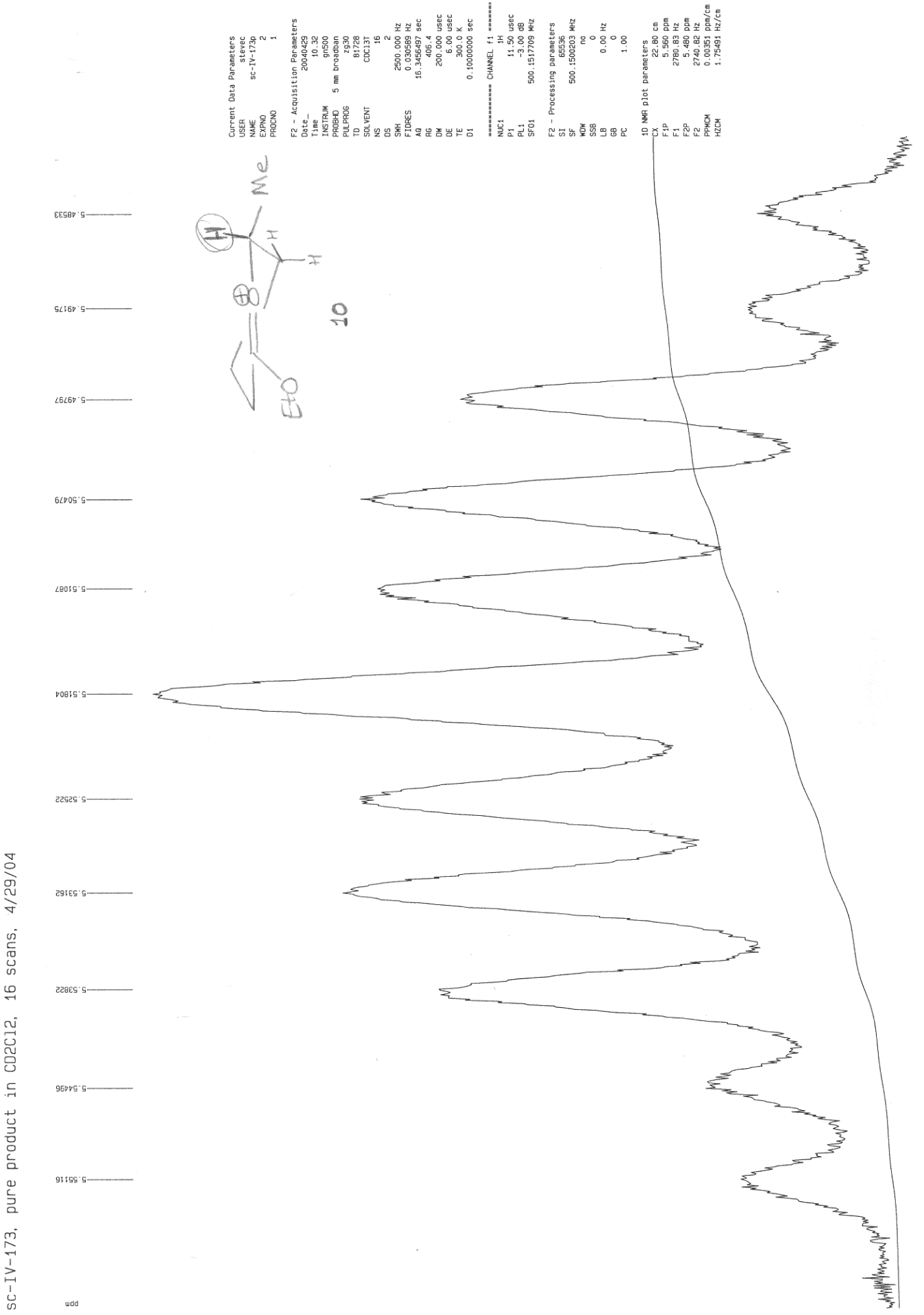

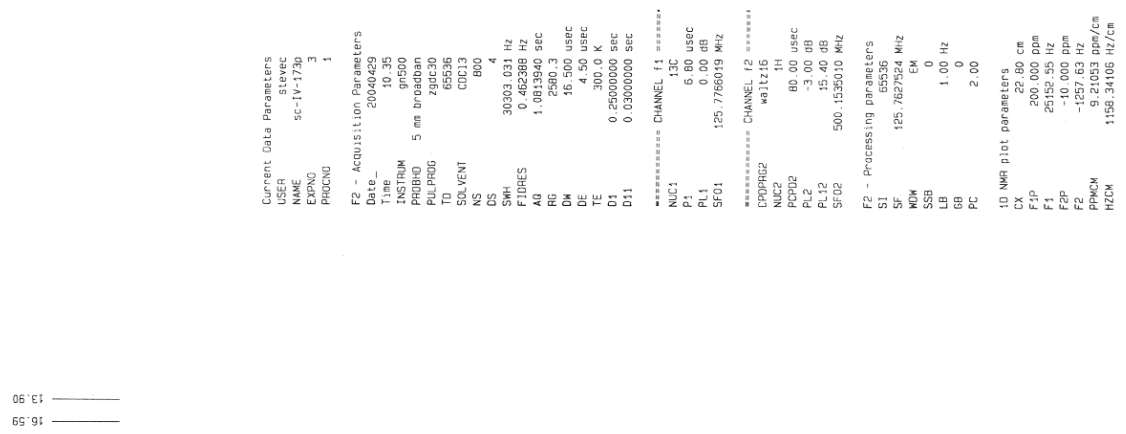

¿द.

${ }_{006282}^{06}=$

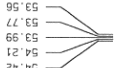

7956
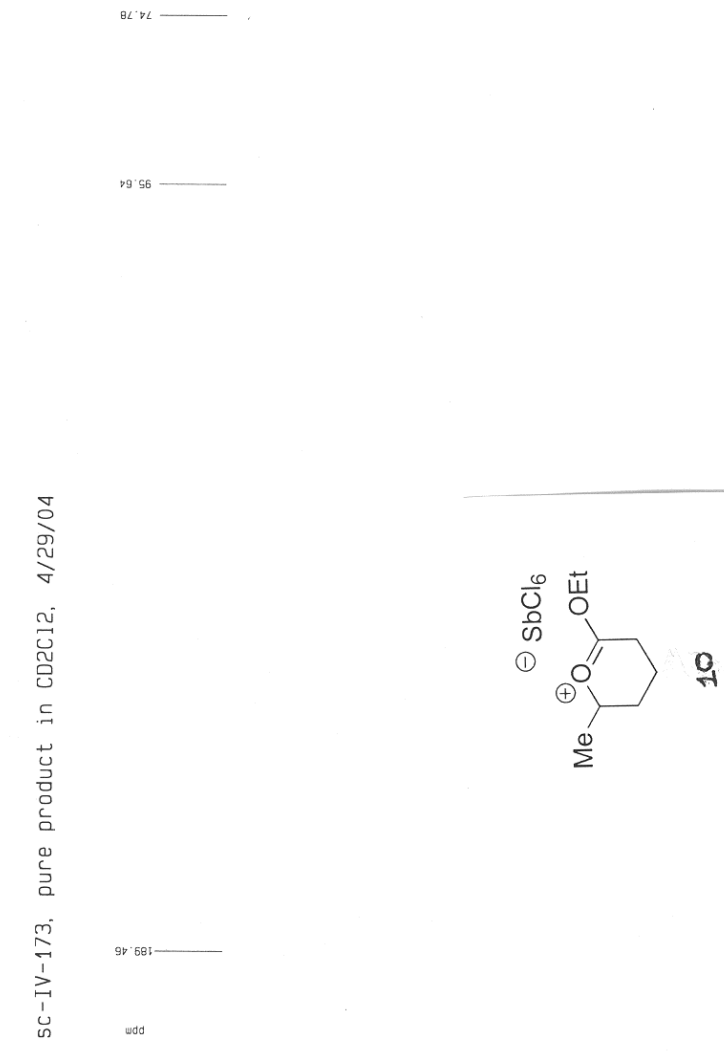


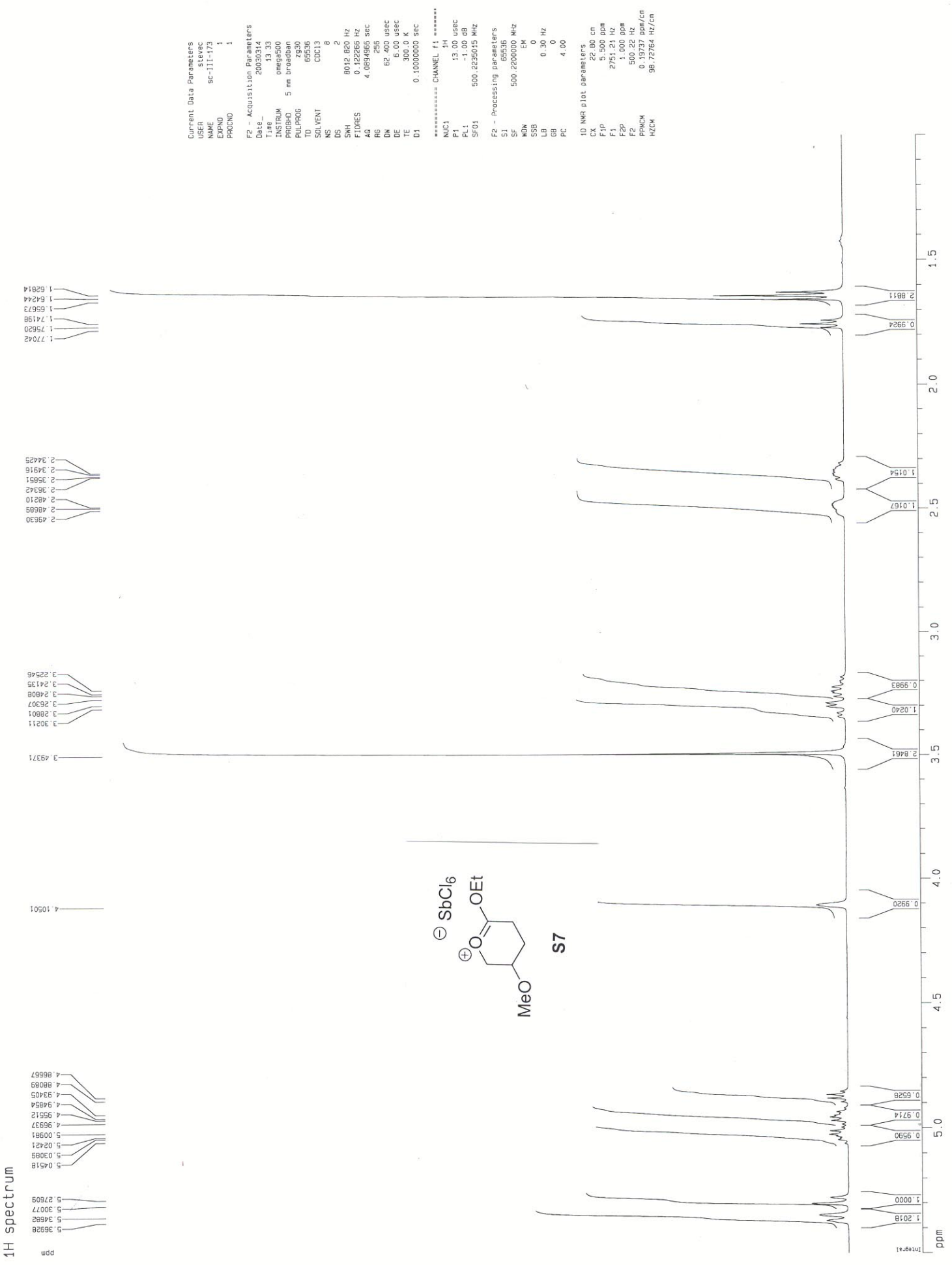



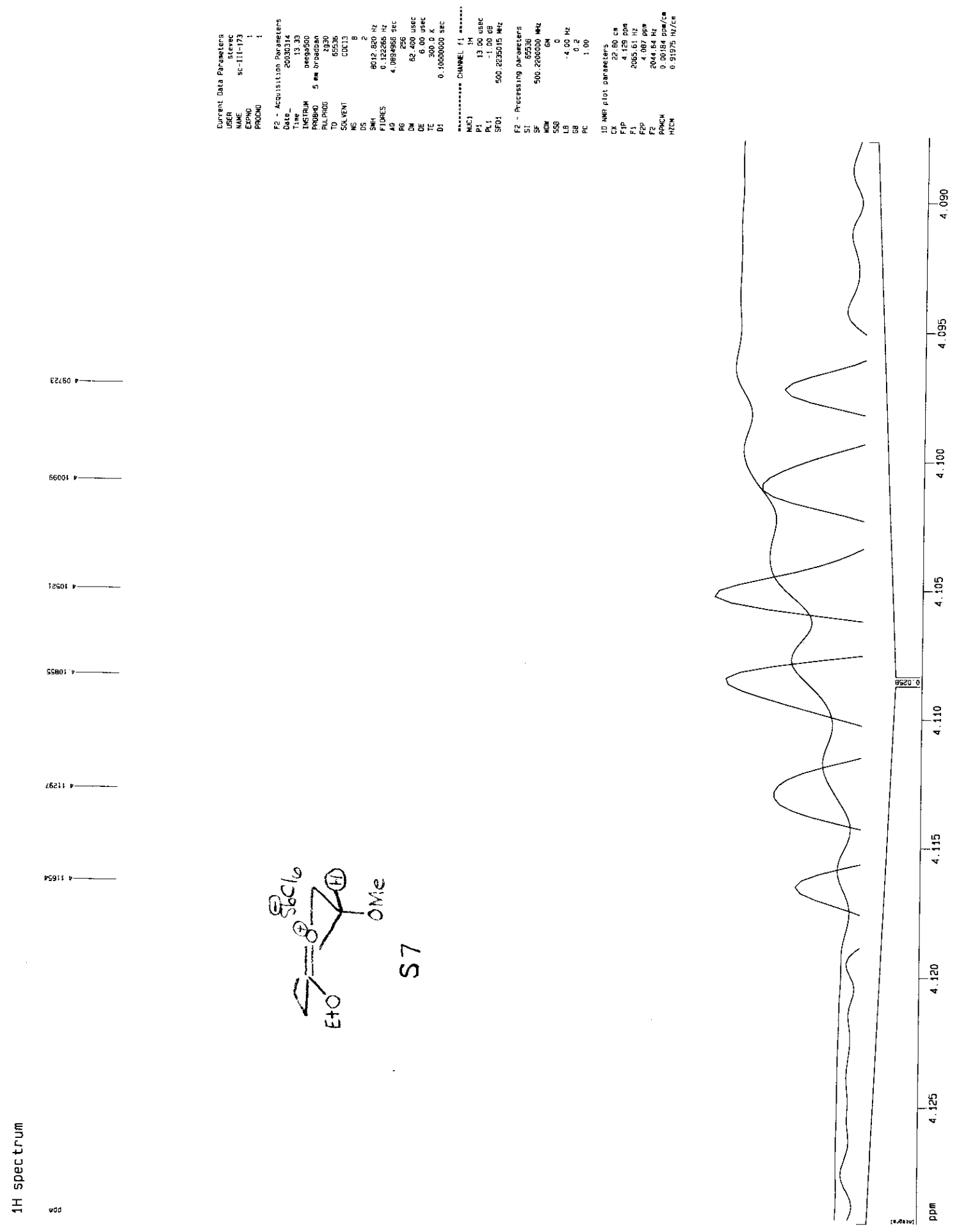

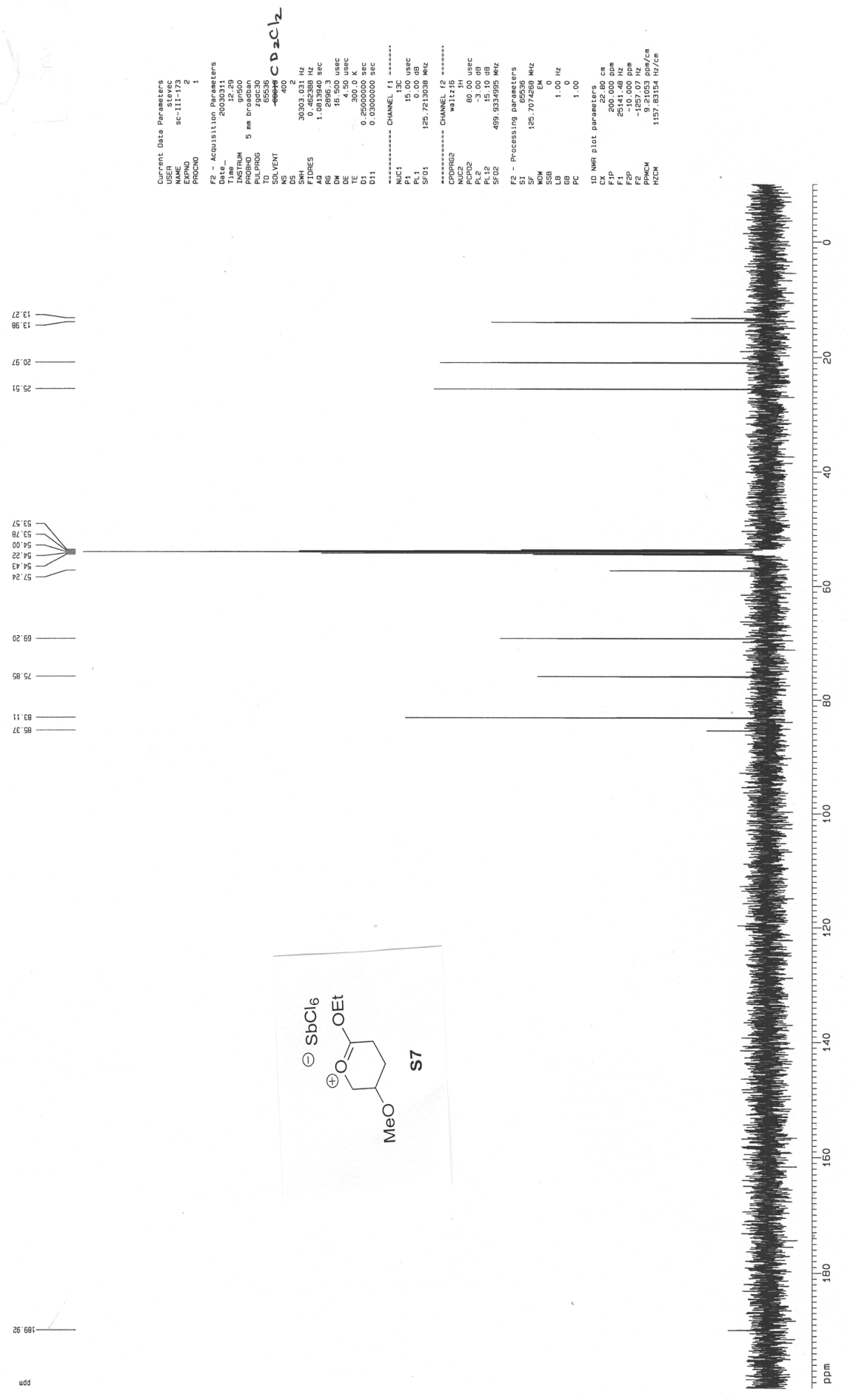

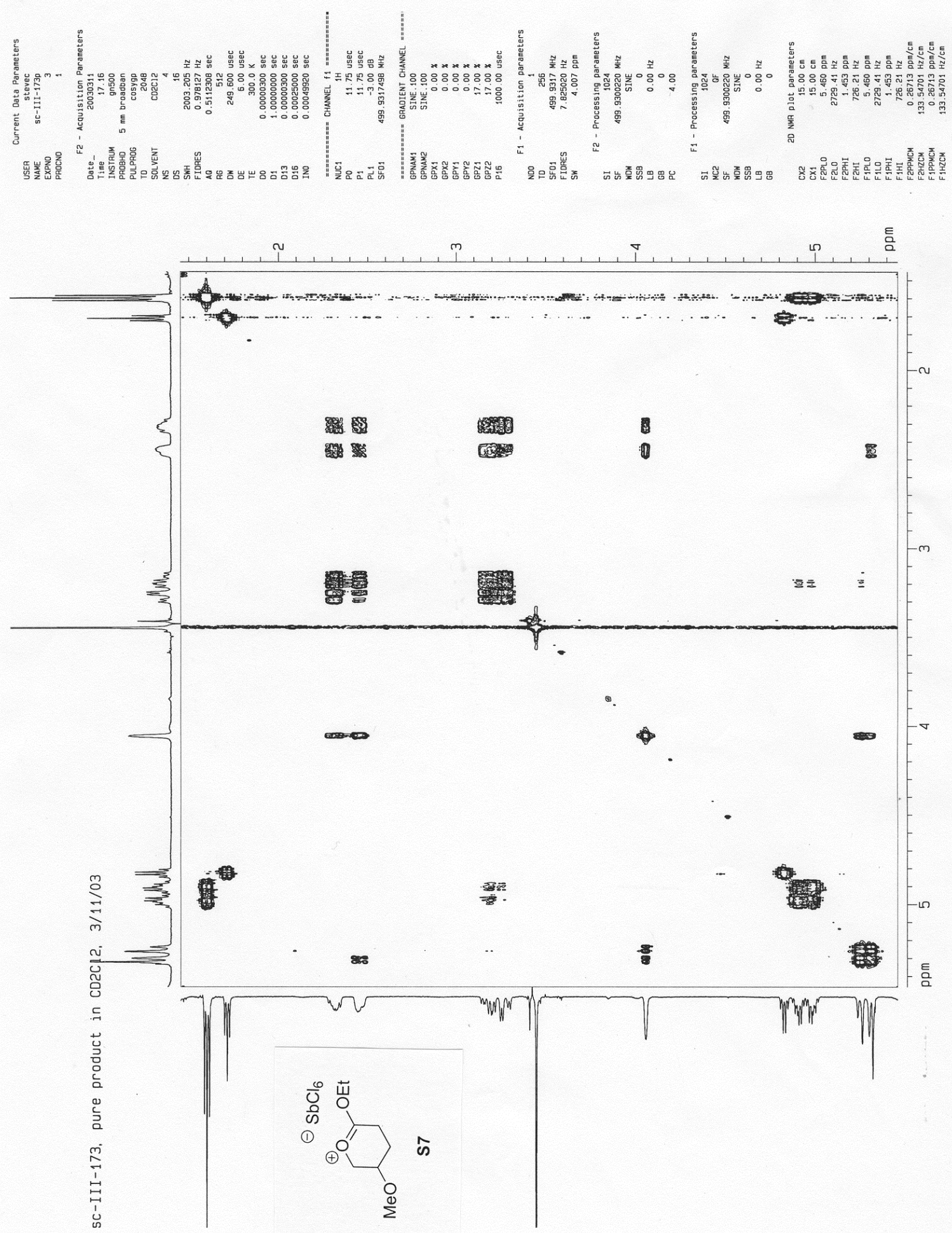

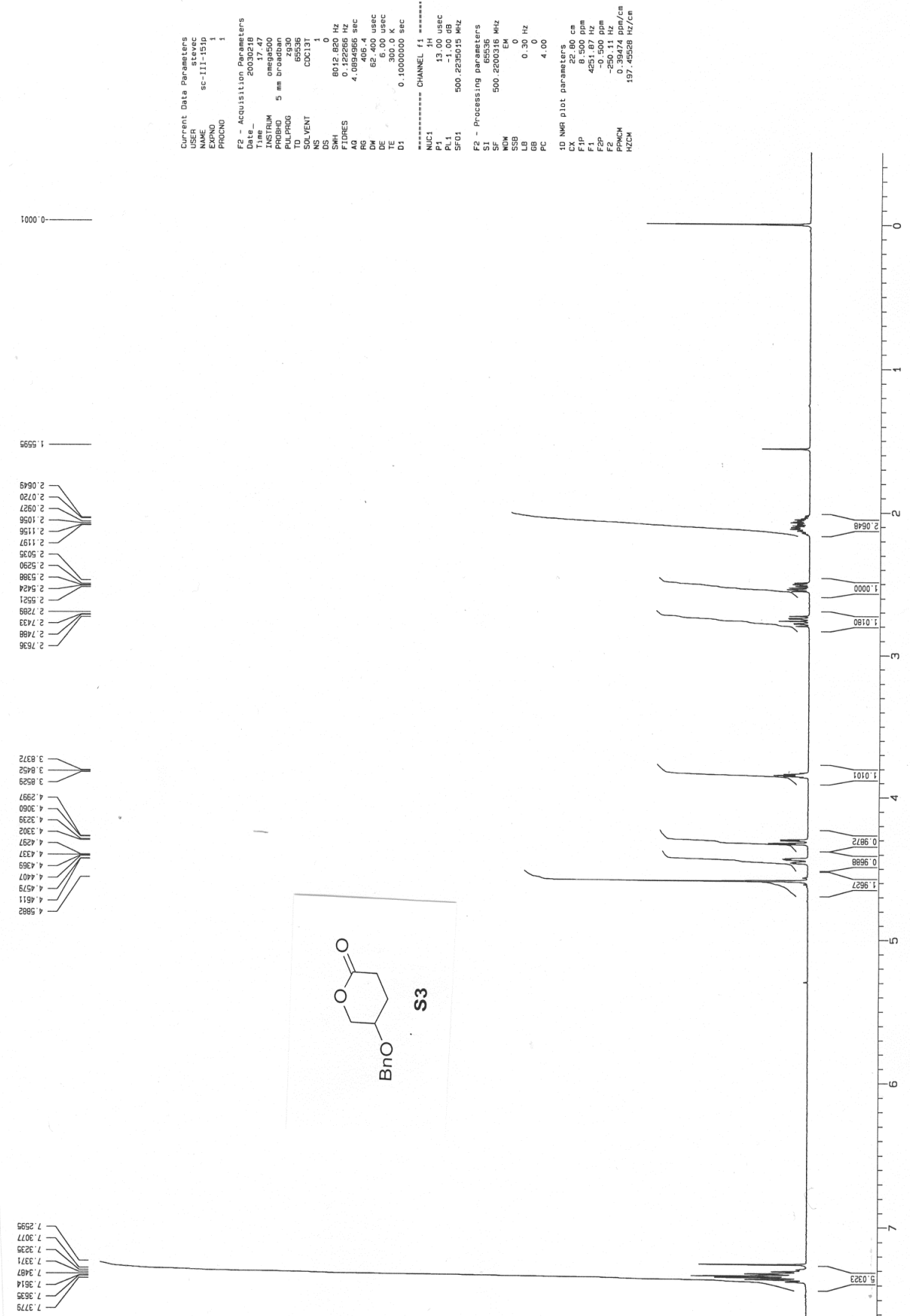

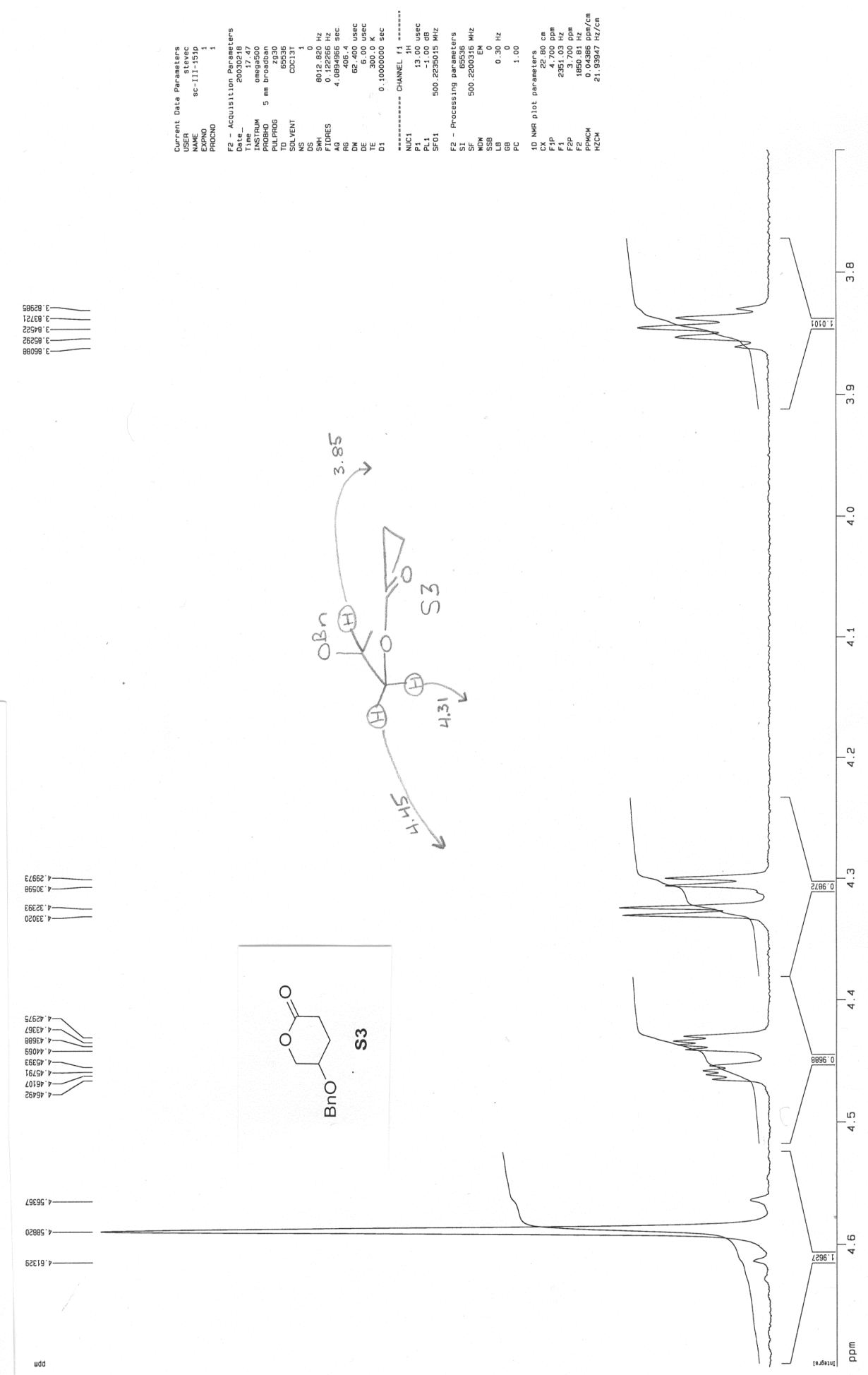

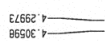

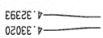
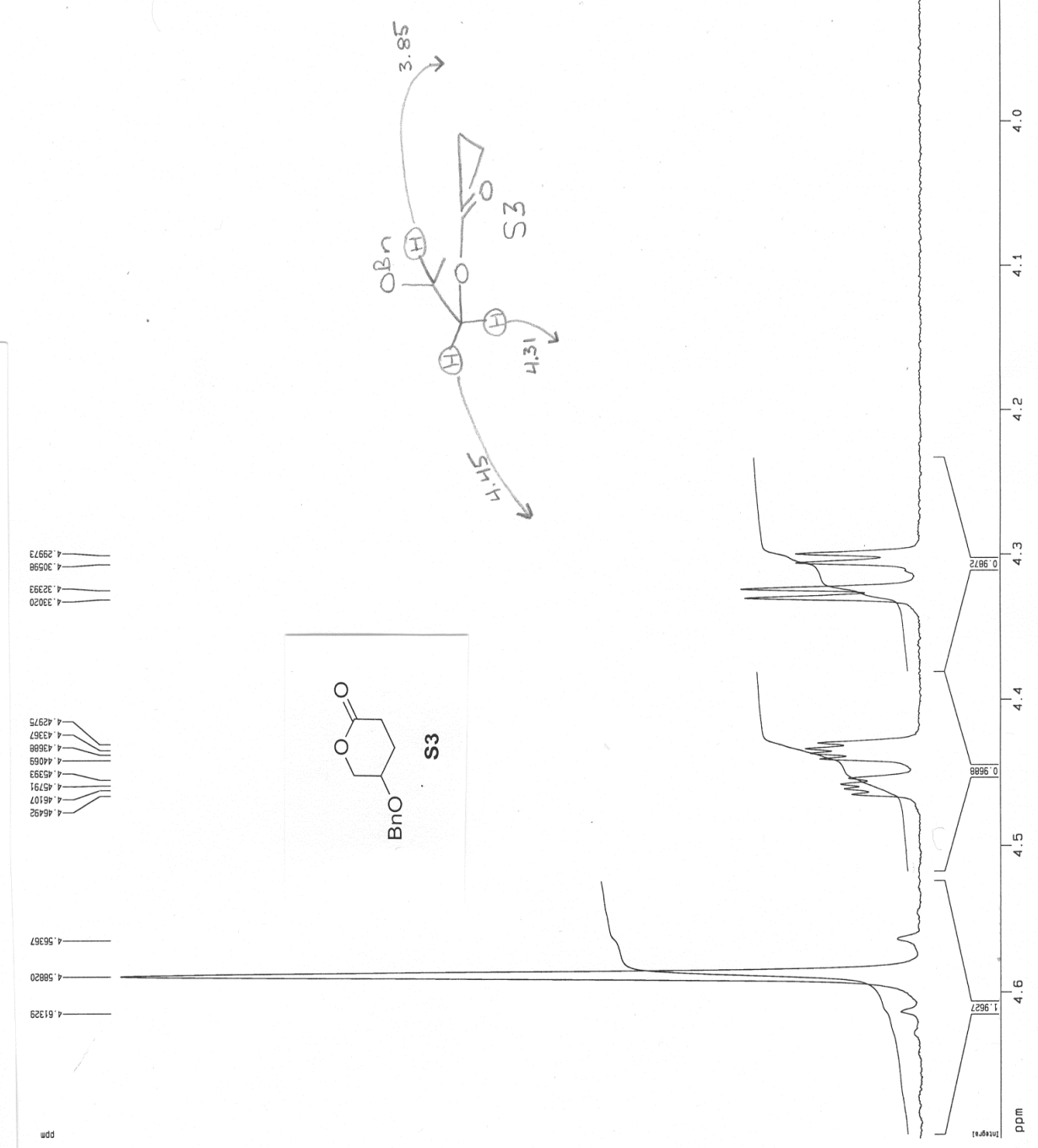

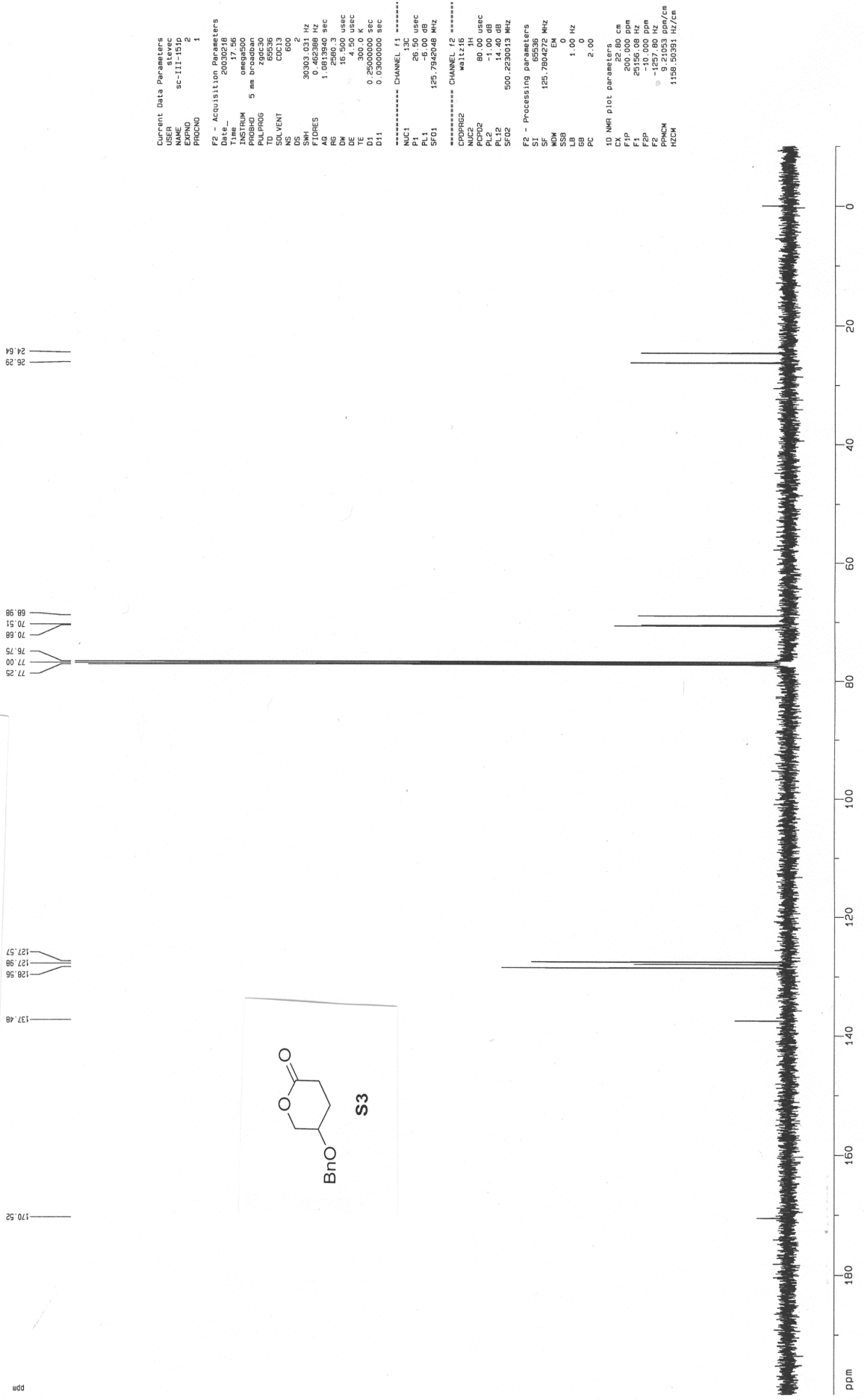

25: OLL -
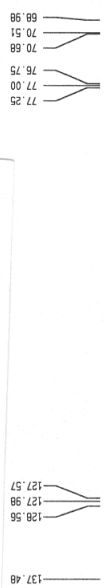


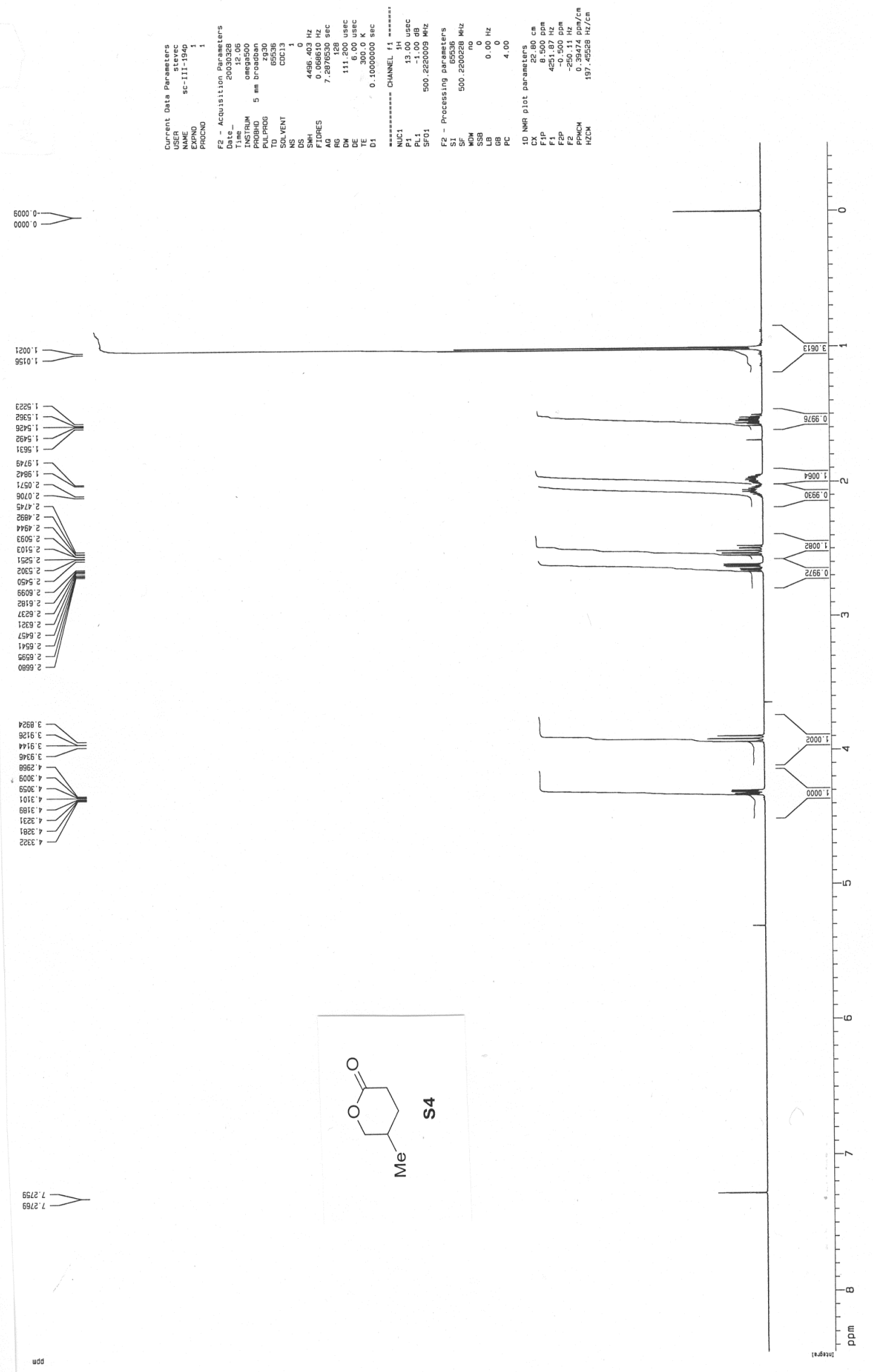



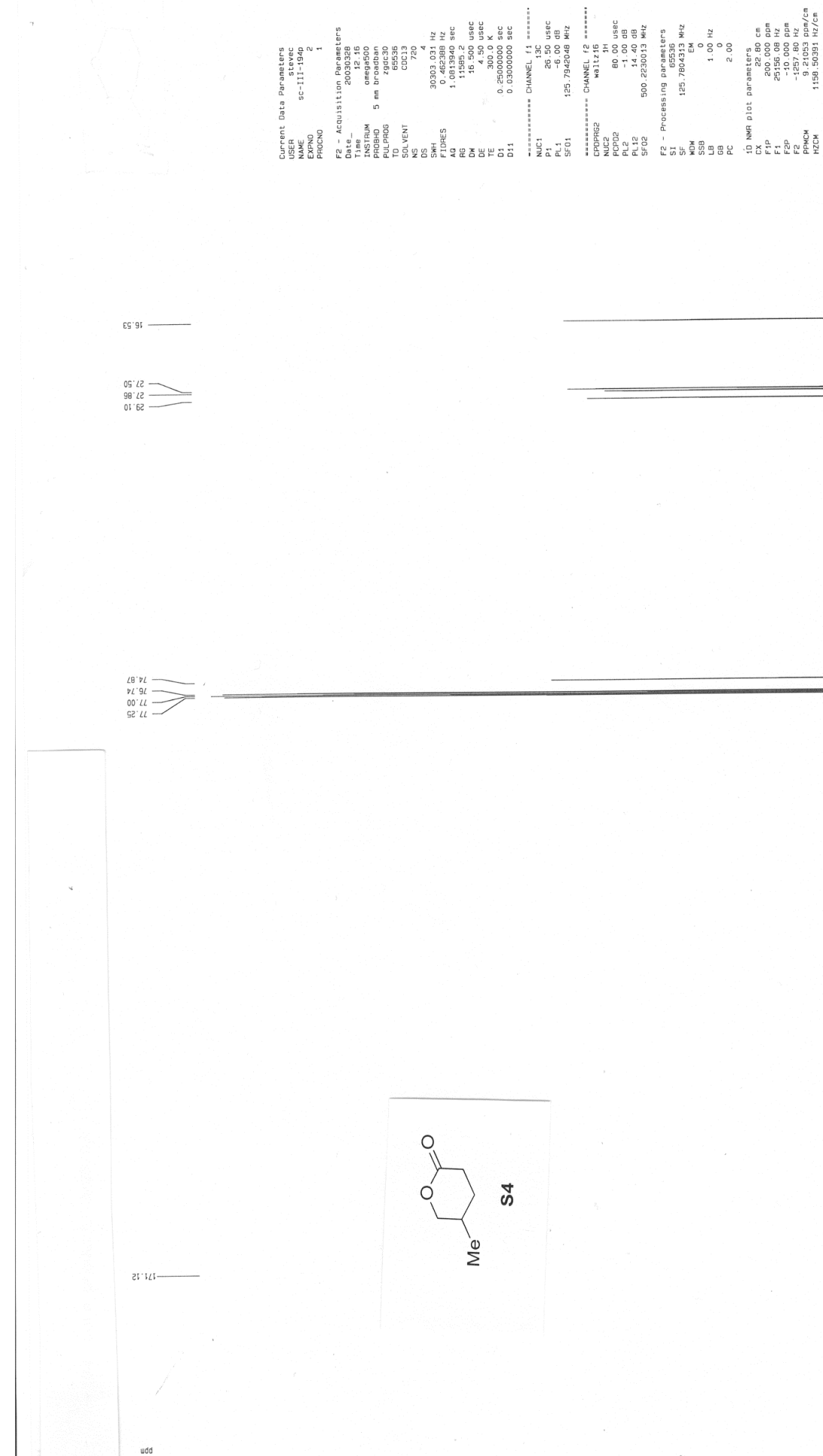

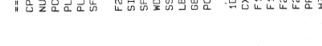

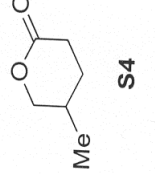



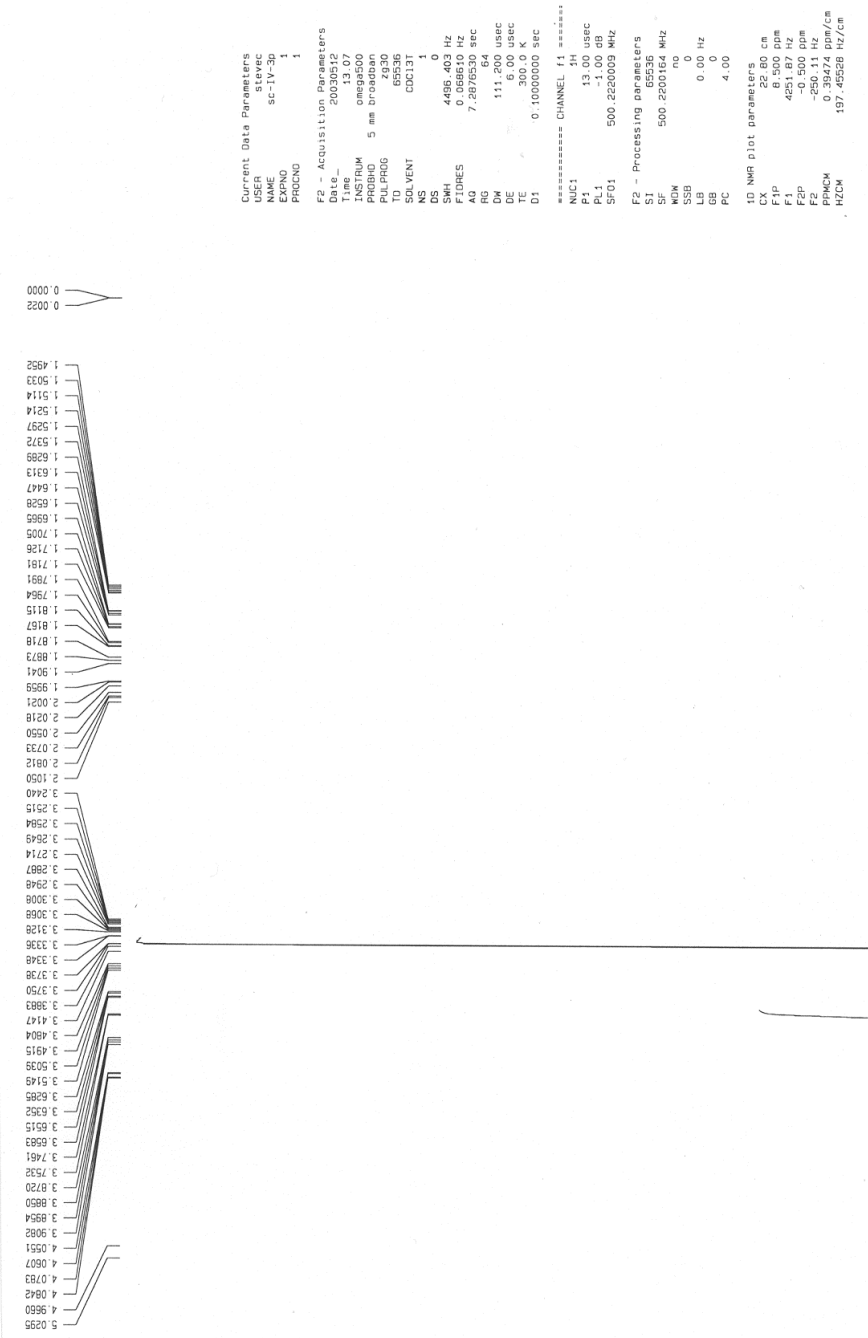

\section{I}

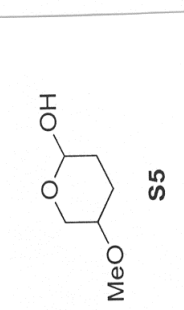

taex:

wo

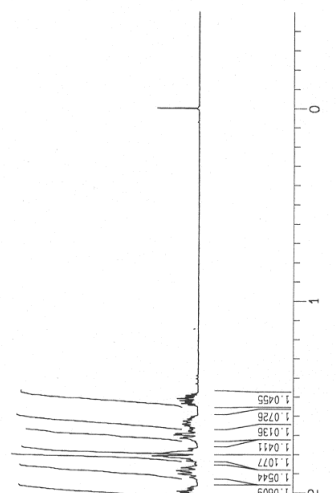



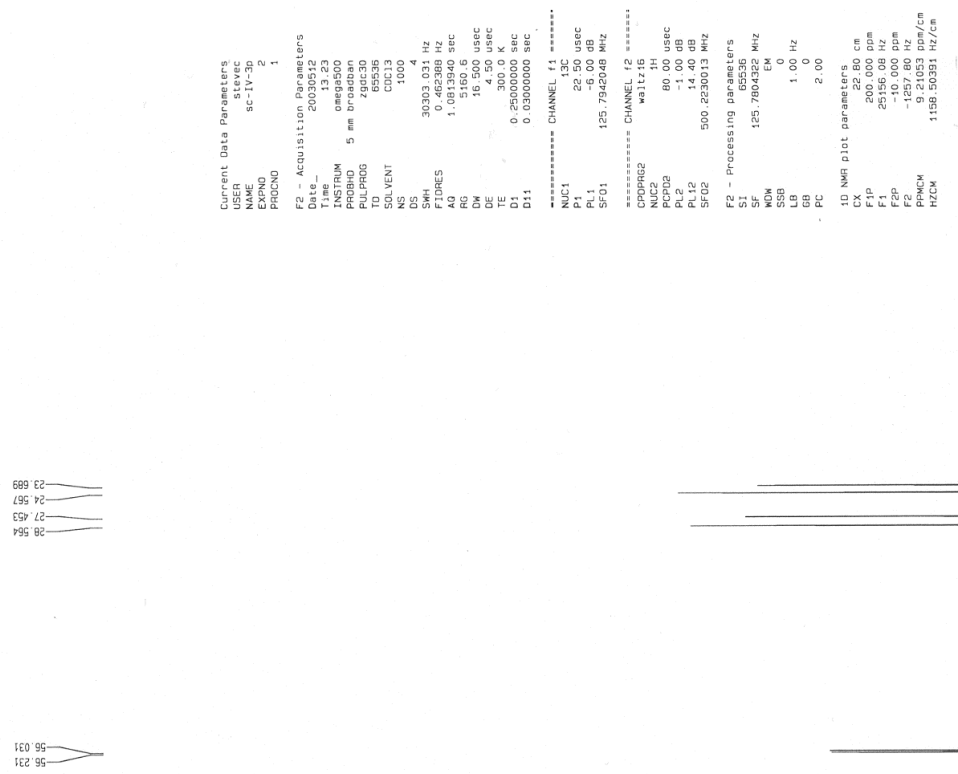

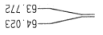

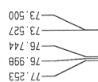

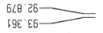

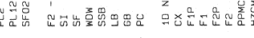

I
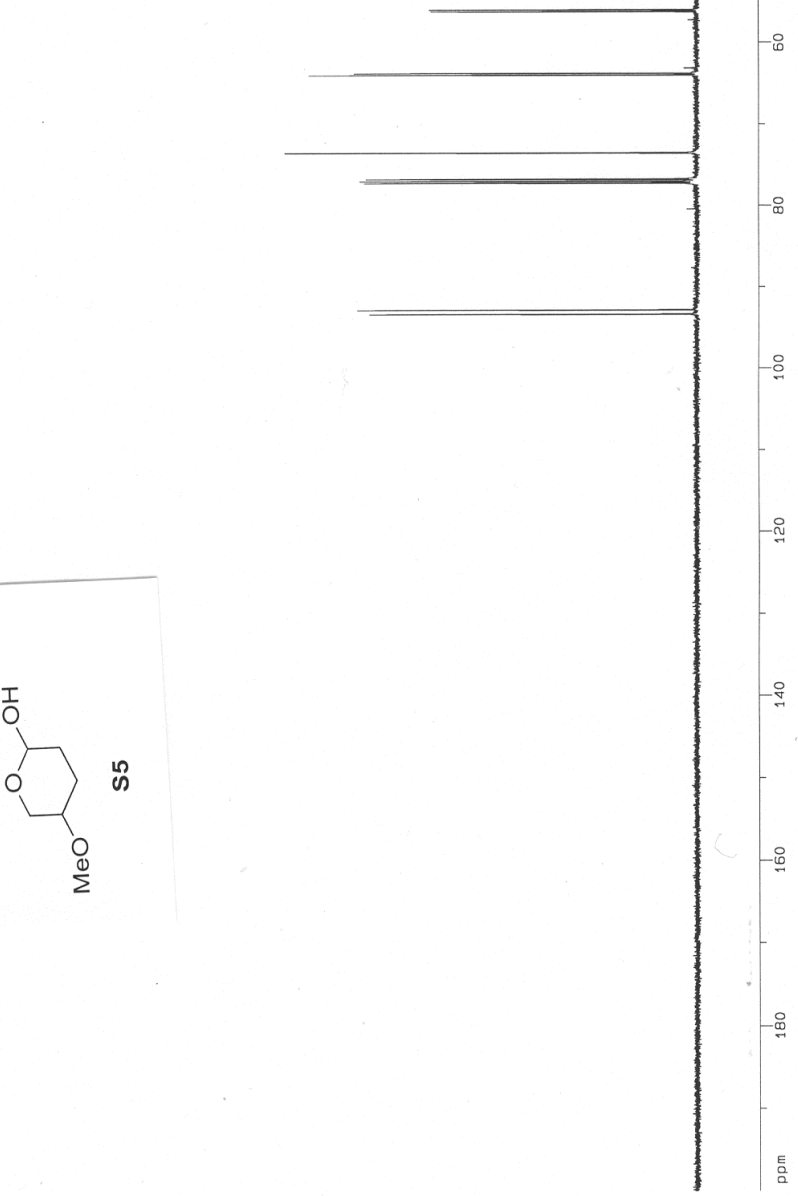

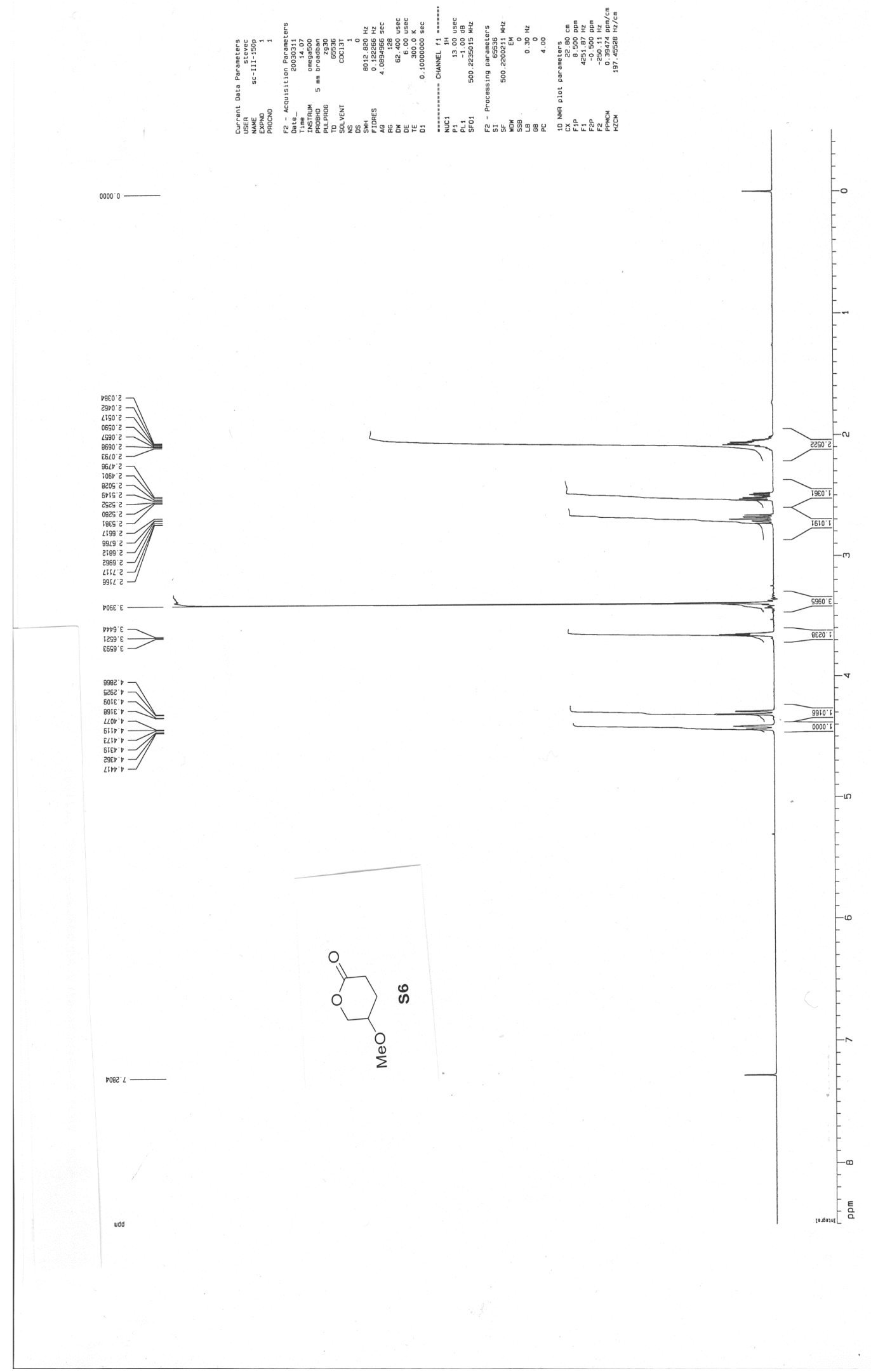

mext

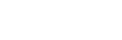



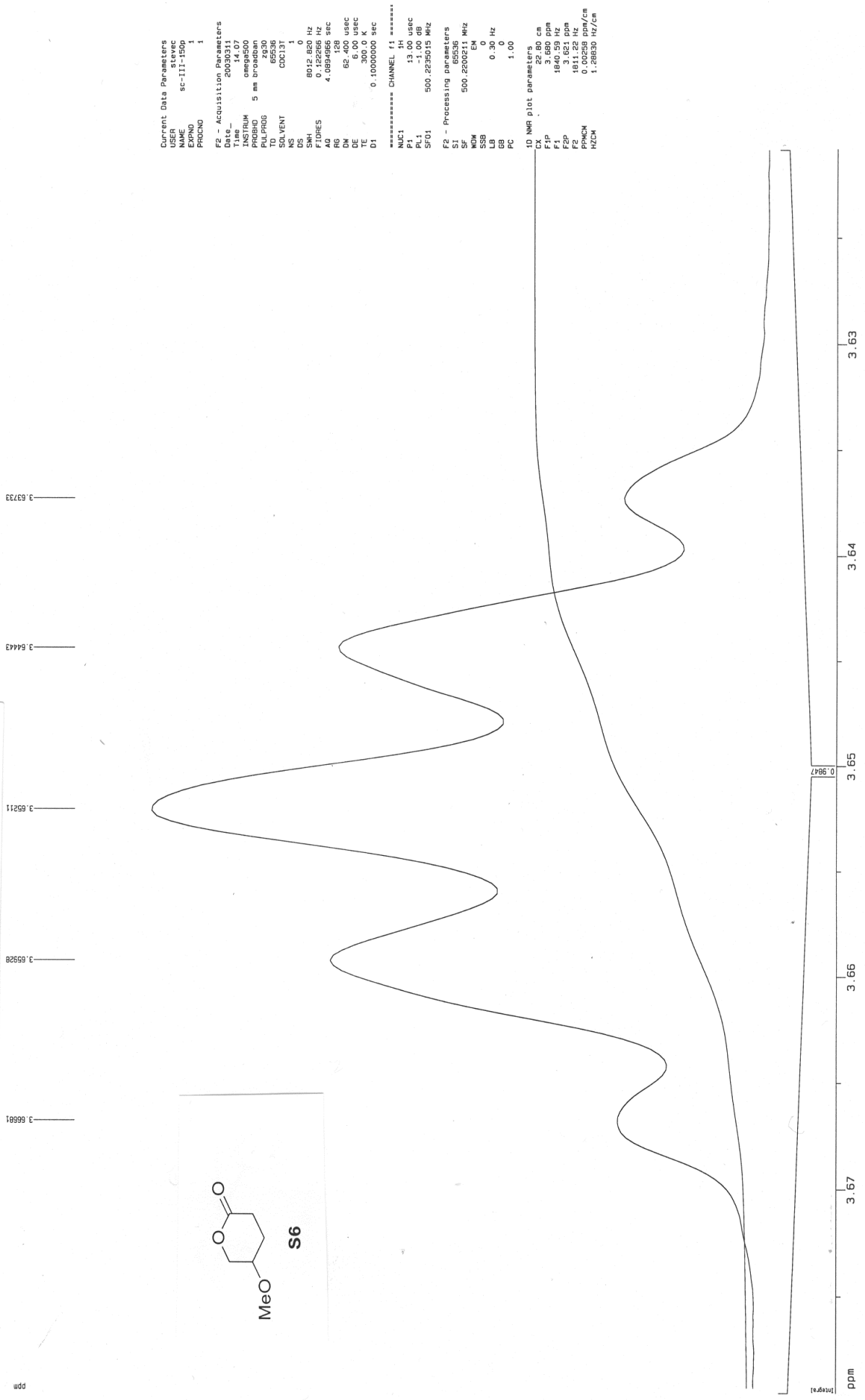

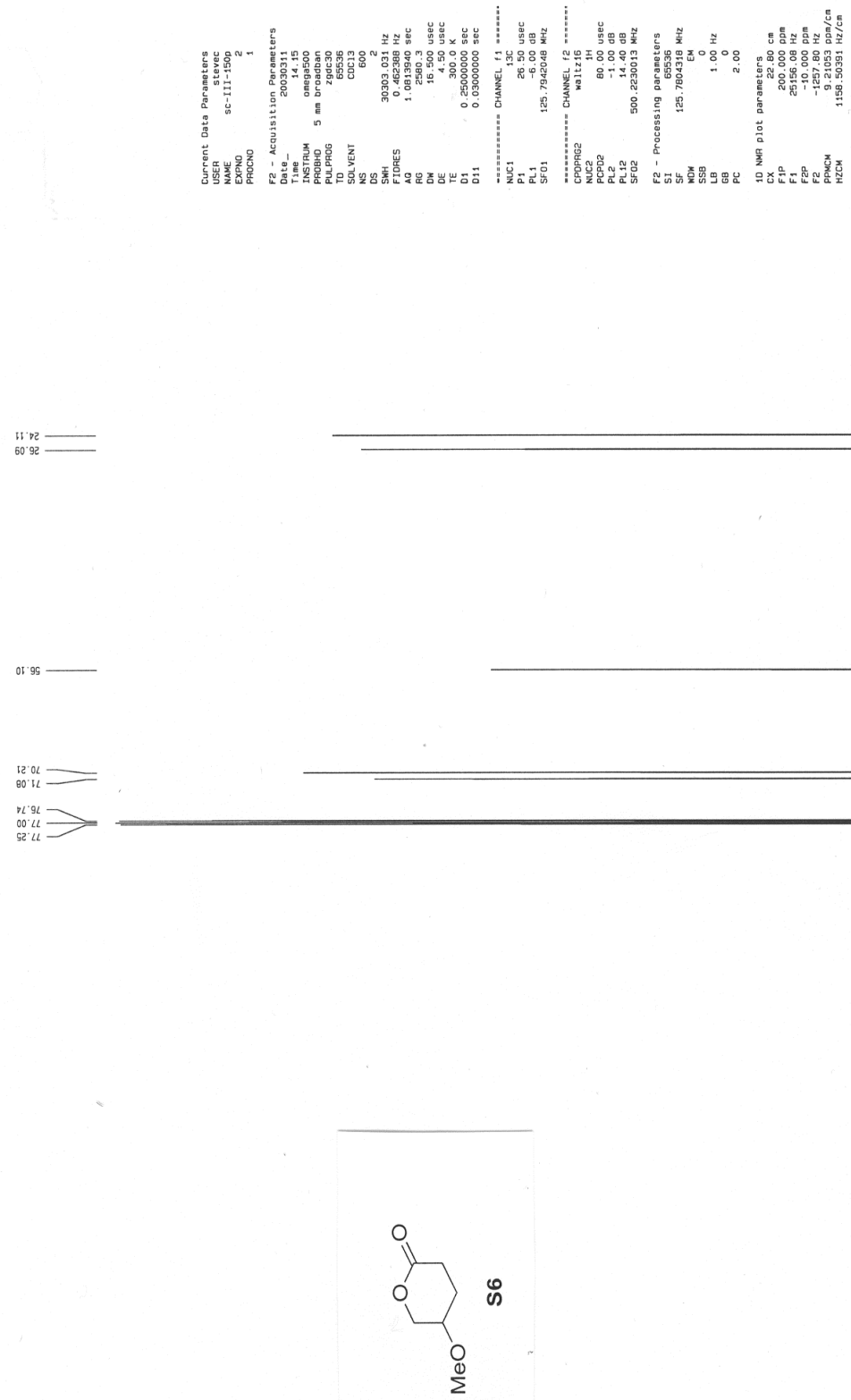

19065

$\sum^{\infty}$ 
